\title{
Fully Atomistic Understanding of the Electronic and Optical Properties of a Prototypical Doped Charge-Transfer Interface
}

Anu Baby, ${ }^{\dagger,}, \triangle$ Marco Gruenewald, ${ }^{\S, \triangle}$ Christian Zwick, ${ }^{\S}$ Felix Otto, ${ }^{\S}$ Roman Forker, ${ }^{\S}$ Gerben van Straaten, ${ }^{\|, \perp}$ Markus Franke, ${ }^{\|, \perp}$ Benjamin Stadtmüller, ${ }^{\#, \mathscr{I}}$ Christian Kumpf, ${ }^{\|, \perp}$

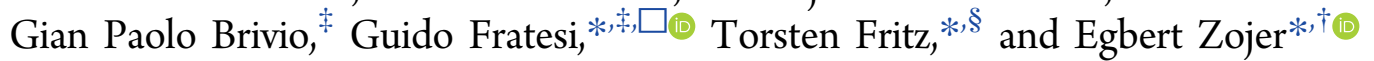

${ }^{\dagger}$ Institute of Solid State Physics, NAWI Graz, Graz University of Technology, Petersgasse 16, 8010 Graz, Austria

${ }^{\ddagger}$ Department of Materials Science, University of Milano-Bicocca, Via R. Cozzi 55, 20125 Milano, Italy

${ }^{\S}$ Institute of Solid State Physics, Friedrich Schiller University Jena, Helmholtzweg 5, 07743 Jena, Germany

"Peter Grünberg Institut (PGI-3), Forschungszentrum Jülich, 52425 Jülich, Germany

${ }^{\perp}$ Jülich Aachen Research Alliance (JARA)-Fundamentals of Future Information Technology, 52425 Jülich, Germany

${ }^{\#}$ Department of Physics and Research Center OPTIMAS, University of Kaiserslautern, 67663 Kaiserslautern, Germany

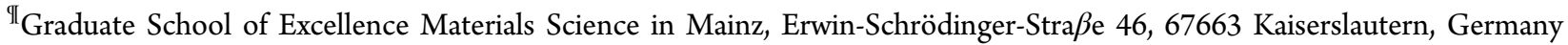

$\square$ Dipartimento di Fisica, Università degli Studi di Milano, Via Celoria 16, 20133 Milano, Italy

\section{Supporting Information}

ABSTRACT: The current study generates profound atomistic insights into doping-induced changes of the optical and electronic properties of the prototypical PTCDA/ $/ \operatorname{Ag}(111)$ interface. For doping $\mathrm{K}$ atoms are used, as $\mathrm{K}_{x} \mathrm{PTCDA} /$ $\operatorname{Ag}(111)$ has the distinct advantage of forming well-defined stoichiometric phases. To arrive at a conclusive, unambiguous, and fully atomistic understanding of the interface properties, we combine state-of-the-art density-functional theory calculations with optical differential reflectance data, photoelectron spectra, and $\mathrm{X}$-ray standing wave measurements. In combination with the full structural characterization of the $\mathrm{K}_{x}$ PTCDA $/ \mathrm{Ag}(111)$ interface by low-energy electron diffraction and scanning tunneling microscopy

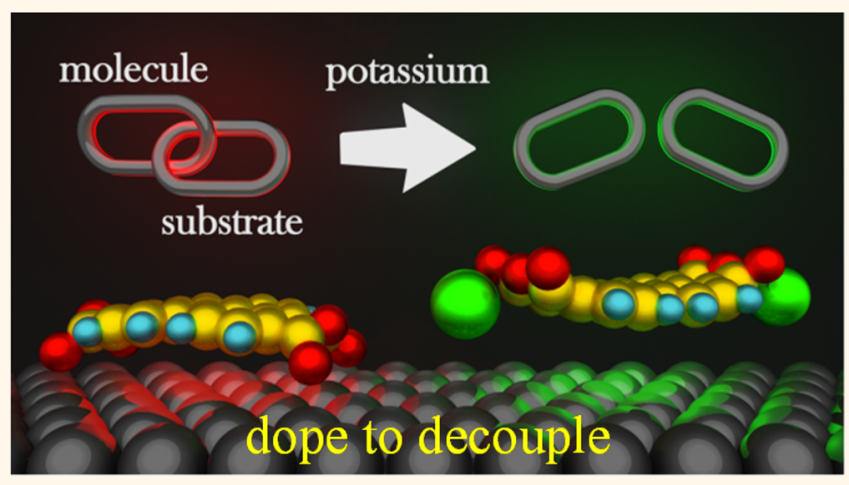
experiments (ACS Nano 2016, 10, 2365-2374), the present comprehensive study provides access to a fully characterized reference system for a well-defined metal-organic interface in the presence of dopant atoms, which can serve as an ideal benchmark for future research and applications. The combination of the employed complementary techniques allows us to understand the peculiarities of the optical spectra of $K_{2} P T C D A / A g(111)$ and their counterintuitive similarity to those of neutral PTCDA layers. They also clearly describe the transition from a metallic character of the (pristine) adsorbed PTCDA layer on $\operatorname{Ag}(111)$ to a semiconducting state upon doping, which is the opposite of the effect (degenerate) doping usually has on semiconducting materials. All experimental and theoretical efforts also unanimously reveal a reduced electronic coupling between the adsorbate and the substrate, which goes hand in hand with an increasing adsorption distance of the PTCDA molecules caused by a bending of their carboxylic oxygens away from the substrate and toward the potassium atoms.

KEYWORDS: metal-organic interface, doping, density-functional theory calculations, X-ray standing wave,

differential reflectance spectroscopy, electronic structure, optical properties

A

lkali-metal-doped organic semiconductor films have demonstrated a wide variety of interesting properties and have been used in numerous applications ranging from superconductivity ${ }^{1-5}$ to hydrogen storage $^{6,7}$ and batteries. ${ }^{8}$ Additionally, it has been shown that alkali-metal
Received: August 16, 2017

Accepted: September 13, 2017

Published: September 13, 2017 
doping can be exploited to tune the spin properties of a hybrid ferromagnetic metal-organic interface. ${ }^{9}$ The charge transfer between alkali-metal atoms and organic molecules also strongly impacts the electronic properties of the latter. ${ }^{10}$ This is highly relevant for carrier injection in electronic devices, ${ }^{11,12}$ as the energy level alignment at the interfaces between the metal electrode and the active organic layer can be tuned by changing the stoichiometry of the dopant-host network. ${ }^{13,14}$ Consequently, alkali-metal doping has been shown to increase the efficiency of organic light-emitting devices. ${ }^{15}$ The dopinginduced charge transfer also significantly influences the optical properties of organic molecules, ${ }^{16}$ where the nature of the formed intragap states depends on the amount of induced charges rather than the type of the dopant used. ${ }^{17}$

Understanding the effect of alkali-metal doping on organic monolayers strongly interacting with metal substrates is particularly challenging. The reasons for that are twofold: First, the structure of the organic adsorbate layer can change significantly in the presence of alkali metal atoms, ${ }^{18,19}$ which can have a crucial impact on both the electronic and optical properties of the interface. Second, one is dealing with a complex interplay of charge transfer and potentially also covalent interactions occurring between the dopants and organic molecules, the dopants and the substrate, and the substrate and the adsorbed organic molecules. In fact, under certain circumstances alkali-metal atoms have been found to decouple adsorbate molecules from metal substrates, ${ }^{20}$ and for the sexiphenyl/ $\mathrm{Cu}(110)$ interface at low doping concentrations they even result in a depopulation of formerly occupied states in the organic layer. ${ }^{21}$ As a consequence, to arrive at a conclusive picture, it is crucial to combine a variety of experimental techniques that provide information on the structural, optical, and electronic properties of the studied system with state-of-the-art quantum-mechanical calculations. The simulations, on one hand, allow an unambiguous interpretation of the experimental observations and, on the other hand, provide microscopic insight into charge-density rearrangements occurring at the interface.

K-doped perylene-3,4,9,10-tetracarboxylic dianhydride (PTCDA) on $\operatorname{Ag}(111)$ is a particularly well-suited system for studying the impact of doping on a molecular monolayer in the presence of interfacial charge transfer with a metal substrate. The reasons for this are (i) that PTCDA on $\operatorname{Ag}(111)$ is undisputedly the best investigated interface between a noble metal surface and an organic semiconductor molecule ${ }^{22-26}$ and (ii) that only specific phases of $\mathrm{K}_{x} \mathrm{PTCDA}$ with well-defined stoichiometries exist on $\operatorname{Ag}(111)$, as known from a previous study: ${ }^{19}$ At comparably low K-doping levels (in the following referred to as $\mathrm{K}_{2} \mathrm{PTCDA} / \mathrm{Ag}(111)$ ), the well-known herringbone structure of PTCDA on $\operatorname{Ag}(111)^{22}$ (see Figure 1a) spontaneously converts into another highly ordered phase with two $\mathrm{K}$ atoms and one PTCDA molecule per adsorbate unit cell. The latter is characterized by a point-on-line (POL) epitaxial relation to the substrate. ${ }^{27}$ The potassium atoms are located between the carboxylic and anhydride oxygen atoms, as shown in the structural model in Figure 1b. At higher doping levels, a second phase transition occurs and the resulting structure is characterized again by rows of $\mathrm{K}$ atoms arranged between rows of PTCDA molecules (Figure 1c). However, in this structural phase, the molecules are oriented essentially perpendicular to the direction of the potassium rows, and there are four potassium atoms per molecule (thus, this phase will be referred to as $\mathrm{K}_{4} \mathrm{PTCDA} / \operatorname{Ag}(111)$ ). No intermediate phases (such as
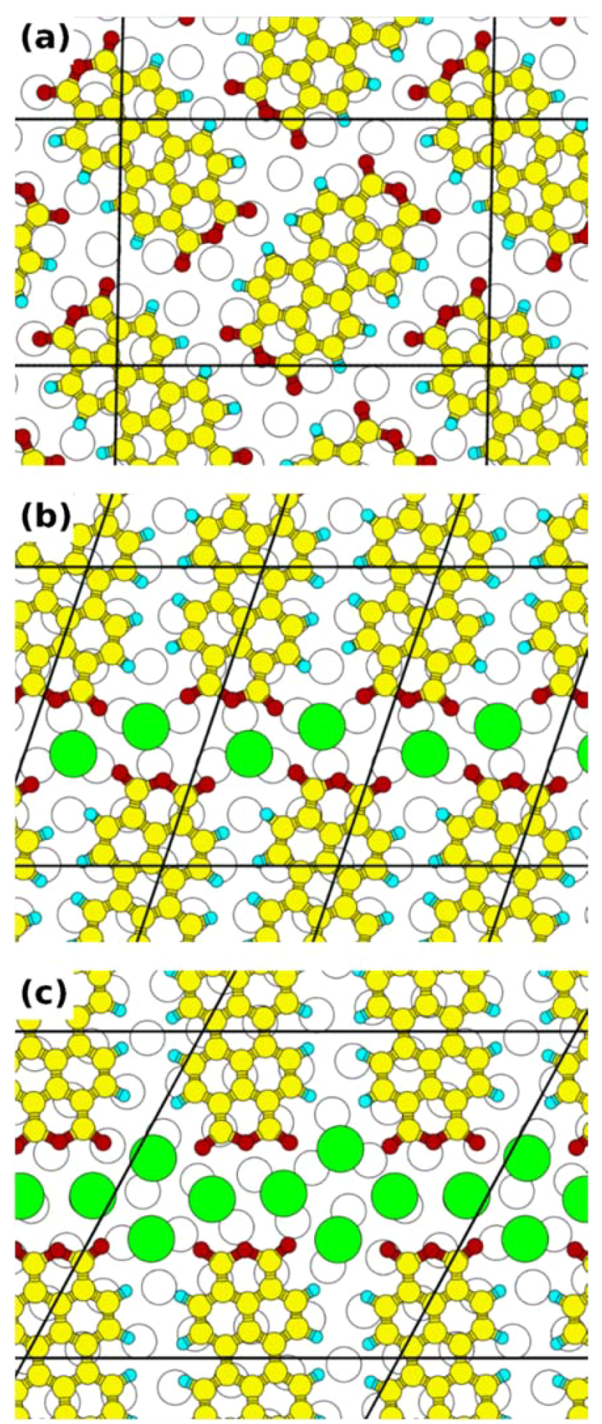

Figure 1. Structures of the (a) PTCDA, (b) $\mathrm{K}_{2} \mathrm{PTCDA}$, and (c) $\mathrm{K}_{4} \mathrm{PTCDA}$ adsorbate layers (studied in the present paper) as previously determined by scanning tunneling microscopy (STM) and distortion-corrected low-energy electron diffraction (LEED) experiments in combination with DFT simulations. ${ }^{19}$ Atoms are depicted with circles colored as follows: yellow for $\mathrm{C}$, blue for $\mathrm{H}$, red for $\mathrm{O}$, white for $\mathrm{Ag}$, and green for $\mathrm{K}$. The parallelograms indicate the surface unit cells of the interface used for the calculations. Only first-layer $\mathrm{Ag}$ atoms are shown; their positions are the ones used in the simulations (including minor distortions of the Ag substrate to generate commensurate structures, $c f$. Methods section.).

$\mathrm{K}_{1}$ PTCDA or $\mathrm{K}_{3}$ PTCDA) have been observed, which significantly eases the interpretation of the obtained spectra. Rather, at intermediate doping levels the above-mentioned stable phases coexist. Only at even higher potassium content has another well-ordered phase been detected, which is, however, unstable and degrades within an hour at room temperature by segregation of $\mathrm{K}$, thereby forming the $\mathrm{K}_{4} \mathrm{PTCDA} / \operatorname{Ag}(111)$ phase with a relatively high defect concentration. ${ }^{19}$

Here we present an in-depth study on how these structural and chemical changes of the adsorbate layer affect its optical and electronic properties. We start with a detailed discussion of the optical properties of the metal-organic interface obtained 
by differential reflectance spectroscopy (DRS) experiments. For the systems studied here, these, however, yield ambiguous results and do not allow arriving at a conclusive understanding of how the electronic structure of the interface changes upon $\mathrm{K}$ doping. To properly understand the observed trends, we therefore performed state-of-the-art simulations relying both on open boundary conditions for free molecules and on periodic ones for adsorbed PTCDA. These are combined with photoelectron spectroscopy and normal incidence X-ray standing wave (NIXSW) studies to obtain the energy level alignment of the occupied frontier orbitals and vertical adsorption distances, respectively. In this way, we are finally able to unambiguously assign the observed optical features and to achieve an in-depth atomistic understanding of the electronic and optical properties of the prototypical interfaces studied here.

\section{RESULTS AND DISCUSSION}

Optical Properties. Monolayer films of pristine and Kdoped PTCDA were grown under ultra-high-vacuum conditions, as described in ref 19 . During the growth of PTCDA and the subsequent deposition of $\mathrm{K}$ we performed DRS experiments in real time to determine the optical properties of the samples. ${ }^{28}$ From the DRS raw data, the imaginary part of the dielectric function of the adsorbate layer $\varepsilon_{\text {ads }}^{\prime \prime}$ was extracted as described in the Methods section and, in greater detail, in the Supporting Information.

The $\varepsilon_{\text {ads }}^{\prime \prime}$ spectra for PTCDA on $\operatorname{Ag}(111)$ at various K doping stages are shown in Figure 2 together with the results of previous DRS investigations for PTCDA on $\mathrm{Au}(111)$ and on mica. ${ }^{29,30}$ The latter are employed here as reference spectra, which can be used to identify the spectral features of the Kdoped PTCDA films. The spectrum of the pristine PTCDA layer on $\operatorname{Ag}(111)$ (black dashed curve in Figure 2a) is significantly broadened and clearly red-shifted compared to that of pristine PTCDA on mica (dashed olive-green curve in Figure 2c). The latter is dominated by largely unperturbed molecular excitations comparable to those of PTCDA dissolved, for example, in dichloromethane $e^{31}$ or isolated in a xenon matrix. ${ }^{32}$ The broadening and shift of the spectrum on $\mathrm{Ag}(111)$ can be interpreted as an indication of a strong (chemical) interaction between the adsorbate layer and the substrate. It is well established that this results in a significant charge transfer from the Ag substrate to the PTCDA molecules and that, consequently, the band derived from the LUMO of PTCDA becomes partially filled ${ }^{24,26,33}$ (vide infra). In that context it is interesting to point out that the maximum of $\varepsilon_{\text {ads }}^{\prime \prime}$ for PTCDA $/ \operatorname{Ag}(111)$ in the displayed energy range is found at essentially the same energy as for the PTCDA monoanion on mica (dotted gray curve in Figure 2c). ${ }^{30}$ This fact will be the subject of a detailed discussion later in this paper.

Upon depositing $\mathrm{K}$, the optical features sharpen and shift to higher energies with the strongest peak fitted at $2.19 \mathrm{eV}$ and a low-energy shoulder remaining at $1.73 \mathrm{eV}$. That shoulder is essentially at the same energy as the peak for PTCDA/ $\mathrm{Ag}(111)$. For an intermediate doping regime corresponding to $\mathrm{K}_{2}$ PTCDA (solid red curve in Figure $2 \mathrm{a}$ ), the spectrum strongly resembles a superposition of the PTCDA on the $\operatorname{Ag}(111)$ spectrum and that of the PTCDA monolayer on $\mathrm{Au}(111)$ (solid orange curve in Figure 2c). Interestingly, for the latter it is well established that the adsorbate layer is essentially charge neutral with no charge transfer from the substrate. ${ }^{24-26,34}$ Thus, the evolution of the optical spectra at intermediate doping

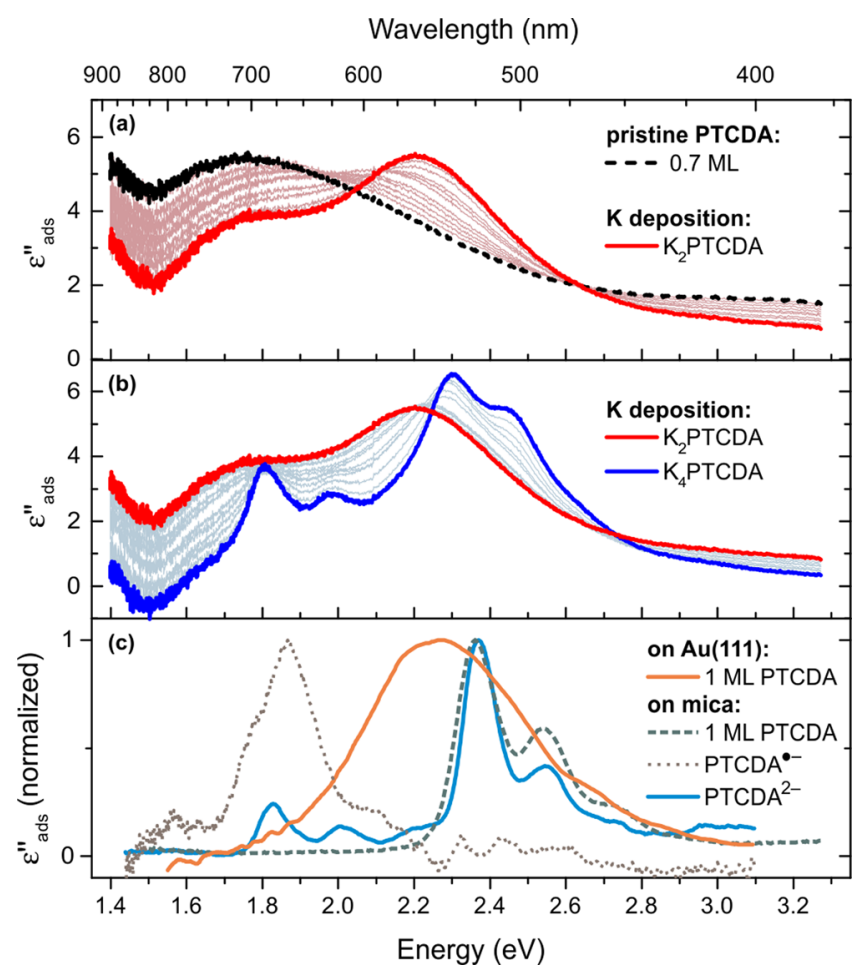

Figure 2. $\varepsilon_{\text {ads }}^{\prime \prime}$ spectra extracted from the DRS raw data of PTCDA on $\operatorname{Ag}(111)$ at various $\mathrm{K}$-doping stages: (a) PTCDA/ $\mathrm{Ag}(111)[d=$ $0.7 \mathrm{ML}$ ], $\mathrm{K}_{2} \mathrm{PTCDA} / \operatorname{Ag}(111)$, and (b) $\mathrm{K}_{4} \mathrm{PTCDA} / \operatorname{Ag}(111)$. Intermediate doping regimes are characterized by a coexistence of the stable PTCDA $/ \operatorname{Ag}(111), K_{2} P T C D A / \operatorname{Ag}(111)$, and $\mathrm{K}_{4}$ PTCDA/Ag(111) phases. $^{19}$ In comparison, panel (c) shows additional spectra for other interfaces containing PTCDA. These are in particular $\varepsilon_{\text {ads }}^{\prime \prime}$ data for 1 ML of PTCDA on Au(111) (from ref 29), 1 ML of neutral PTCDA, PTCDA monoanions (PTCDA ${ }^{\circ-}$ ), and PTCDA dianions $\left(\mathrm{PTCDA}^{2-}\right)$, where PTCDA was deposited on mica and subsequently doped with potassium to produce the charged species (from ref 30 ). The $\varepsilon_{\text {ads }}^{\prime \prime}$ spectra in (c) are smoothed and normalized to their most intense feature.

levels seems to imply that the deposited potassium oxidizes rather than further reduces the PTCDA layer, which contradicts chemical intuition. Exactly this behavior (namely, a reduction of the electron density on the adsorbate due to alkali-metal deposition) has, however, been observed by angle-resolved ultraviolet photoelectron spectroscopy (ARUPS) at low Csdoping concentrations for the sexiphenyl $/ \mathrm{Cu}(110)$ interface due to a Cs-induced decoupling of the adsorbate from the substrate. $^{21}$

For the present interface the scenario of a K-induced oxidation is called into question by the evolution of the spectra upon additional $\mathrm{K}$ deposition depicted in Figure 2b: For $\mathrm{K}_{4}$ PTCDA (solid blue curve) one observes a further blue-shift and sharpening of the spectral features with a clearly resolved double-peak structure of the main maxima at 1.80 and $2.29 \mathrm{eV}$ and their corresponding vibronic replica on the high-energy sides (vide infra). The final spectrum is strongly reminiscent of that of the PTCDA dianion on mica (solid blue curve in Figure 2c). ${ }^{30}$ This observation would be indicative of the a priori expected further reduction of the PTCDA layer by K doping (a trend also observed when increasing the Cs dose for the abovementioned sexiphenyl/ $\mathrm{Cu}(110)$ interface $\left.{ }^{21}\right)$. Considering the increasingly sharper features upon $\mathrm{K}$ deposition, this chemical 
reduction would actually go hand in hand with an electronic decoupling of the adsorbate layer from the $\operatorname{Ag}(111)$ substrate.

To resolve the conundrums arising from interpreting the DRS data (viz., the reduction $v$ s oxidation of the PTCDA layer on $\mathrm{Ag}(111)$ upon $\mathrm{K}$ deposition) and to unambiguously identify the actual electronic structure of the interface, we performed simulations based on dispersion-corrected density-functional theory employing both periodic boundary conditions (when modeling extended interfaces) and open boundary conditions (when simulating molecular excitations). These simulations are backed up by additional ultraviolet photoelectron spectroscopy (UPS) and NIXSW experiments.

Density of States. A first quantity to analyze in order to understand how charges are rearranged upon $\mathrm{K}$ doping is the calculated total density of states projected onto the adsorbate layer (i.e., the PDOS) for the different cases (see Figure $3 a-d$ ). Figure 3a shows the PDOS of gas phase PTCDA; the PDOS of PTCDA upon adsorption on $\mathrm{Ag}(111)$ can be seen in Figure $3 \mathrm{~b}$.
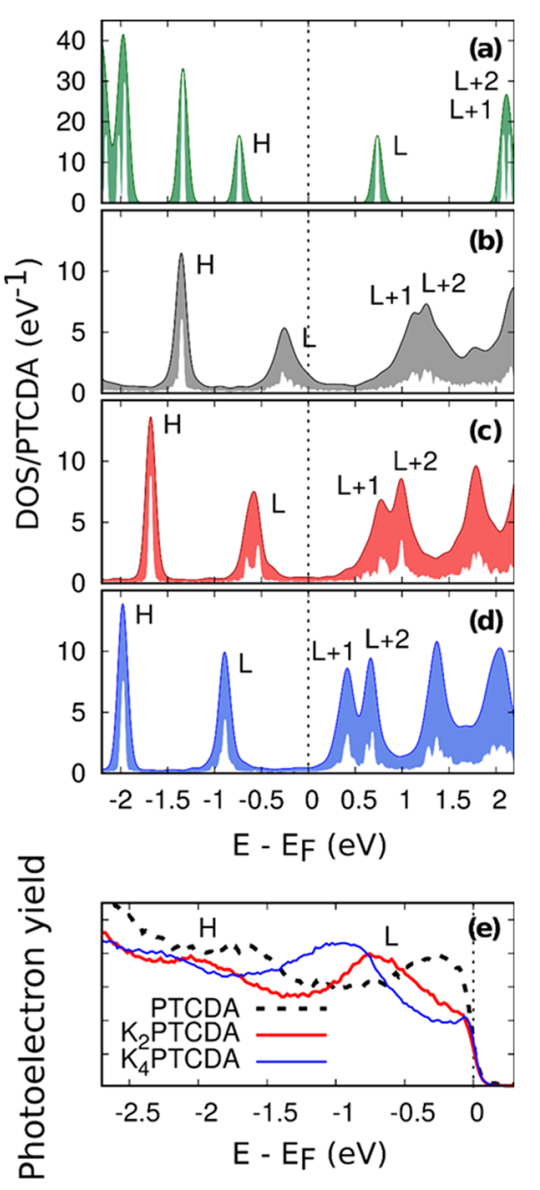

Figure 3. (a) Gas phase density of states of PTCDA (green) and density of states projected onto the adsorbate layer for (b) PTCDA/Ag(111) (black), (c) $\mathrm{K}_{2}$ PTCDA/Ag(111) (red), and (d) $\mathrm{K}_{4} \mathrm{PTCDA} / \mathrm{Ag}(111)$ (blue). We did not include $\mathrm{K}$ in the PDOS as it had minimal influence on the plots. The curves with areas shaded in different colors have been obtained employing a Gaussian broadening of $0.11 \mathrm{eV}$ (FWHM). The PDOS shaded in white has been plotted using a reduced broadening of $0.02 \mathrm{eV}$ (FWHM), and its magnitude has been reduced by a factor of 5 . HOMO, LUMO, $\mathrm{LUMO}+1$, and LUMO +2 of PTCDA are marked as "H", "L", "L+1", and " $L+2$ ", respectively. Panel (e) comprises the experimental UPS data at various doping stages (black: PTCDA/Ag(111), red: $\mathrm{K}_{2} \mathrm{PTCDA} / \mathrm{Ag}(111)$; and blue: $\mathrm{K}_{4} \mathrm{PTCDA} / \operatorname{Ag}(111)$ ).
The partial filling of the LUMO-derived band is clearly observed in the simulations and is also confirmed by the UPS experiments (Figure 3e). This indicates a metallic nature of the adsorbate layer (vide supra). ${ }^{24,26,34}$ Note that in the following we will label bands by the names of the orbitals in the neutral molecule that they are derived from. This is done to avoid confusions arising from using the same names for symmetryinequivalent bands just as a consequence of different degrees of charging.

Interestingly, especially the LUMO, LUMO+1, and $\mathrm{LUMO}+2$ derived bands for the PTCDA/Ag(111) interface broaden significantly compared to the gas phase molecule. The resulting peak width is a measure for the level of hybridization of the electronic states of the adsorbed molecules with the substrate continuum and for the coupling between molecules in the adsorbate layer (note that no vibronic finestructure is considered in the present calculations). A complication when quantifying the peak widths arises from the fact that all plotted DOSs contain an extrinsic Gaussian broadening, which is necessary to avoid artifacts due to the finite number of $\mathrm{Ag}$ layers in the slabs (instead of using a semi-infinite model for the Ag surface $)^{35}$ and due to the finite $k$-point sampling ( $c f$., Methods section). Nevertheless, applying the procedure described in detail in the Supporting Information, one can obtain an estimate for the intrinsic line width. In this way, for the LUMO-derived band in PTCDA/ $\operatorname{Ag}(111)$ in Figure $3 b$ a value of $0.26 \mathrm{eV}$ is obtained for the FWHM.

The electron transfer from the substrate to the adsorbate for PTCDA/ $\operatorname{Ag}(111)$ is also confirmed by the net charges residing on the PTCDA layer as derived from both a Löwdin- ${ }^{36}$ and a Bader-type $^{37}$ charge partitioning, the latter determined by means of a different plane-wave code in ref 19. Both approaches provide equivalent results, namely, the transfer of approximately one electron per PTCDA molecule (see Table 1). The

Table 1. Löwdin Charges (in Units of the Elementary Charge, $e$ ) of the PTCDA $/ \operatorname{Ag}(111)$ Interface at Various Stages of Doping ${ }^{a}$

\begin{tabular}{lccc}
\multicolumn{1}{c}{ system } & $\rho_{\mathrm{K}} / e$ & $\rho_{\mathrm{PTCDA}} / e$ & $\rho_{\mathrm{Ag}} / e$ \\
PTCDA $/ \operatorname{Ag}(111)$ & & $-1.1(-1.1)$ & $+1.1(+1.1)$ \\
$\mathrm{K}_{2} \mathrm{PTCDA} / \operatorname{Ag}(111)$ & $+0.7(+0.8)$ & $-1.3(-1.7)$ & $-0.1(+0.1)$ \\
$\mathrm{K}_{4} \mathrm{PTCDA} / \operatorname{Ag}(111)$ & $+0.6(+0.8)$ & $-1.4(-1.9)$ & $-1.0(-1.3)$
\end{tabular}

${ }^{a}$ Values for potassium are given per $\mathrm{K}$ atom; those for PTCDA and Ag per PTCDA molecule. As partitioning into atomic and molecular charges is not an unambiguous process, we also report (in parentheses) values obtained by the alternative partitioning into Bader charges (from the Supporting Information of ref 19).

seeming discrepancy between this value and the significantly above $50 \%$ filling of the LUMO-derived band (Figure 3) can be resolved by considering that at interfaces between acceptors bearing functional groups that strongly interact with the substrate one observes a delicate balance between charge forward and backward donation. In such a situation, the transfer of electrons to the LUMO is partially compensated by a backtransfer of electrons from the functional groups to the substrate involving strongly hybridizing molecular orbitals. ${ }^{38}$ This effect has been described previously also for the PTCDA/Ag(111) interface. $^{26,39}$

Another observation for PTCDA adsorbed on the $\operatorname{Ag}(111)$ surface is that the (peak-to-peak) HOMO-LUMO gap is reduced by $0.4 \mathrm{eV}$ compared to an isolated PTCDA molecule 
(Figure 3a). This is not due to screening effects at the metal surface, as these are not accounted for when merely looking at Kohn-Sham energies at the PBE level. It is also not related to the monolayer formation, as for a hypothetical free-standing monolayer we observe the same gap as for the isolated molecules (again bearing in mind that screening for excited states is not included in a ground-state calculation). Rather, the reduction of the gap can be interpreted as a consequence of the strong coupling between electronic and nuclear degrees of freedom in conjugated organic materials and the resulting molecular distortions in the charged molecules. ${ }^{40}$

Upon $\mathrm{K}$ doping all features in the calculated PDOS shift to higher binding energies and the LUMO-derived band becomes completely filled, resulting in no states at the Fermi level. This means that there is a metal-to-semiconductor phase transition of the PTCDA monolayer adsorbed on $\operatorname{Ag}(111)$ upon $\mathrm{K}$ doping, a phenomenon commonly observed when interfaces containing organic semiconductors are doped with alkali metals. ${ }^{13,18,41}$ This is consistent with the corresponding charges on the molecule (Table 1), which clearly show that the electron density on the molecule is further increased. The extent to which this is also a consequence of a reduced back-donation from deeper-lying orbitals involving the carboxylic oxygens is difficult to quantify, but the changes in adsorbate geometry suggest that this effect is also of some relevance. Interestingly, the width of the LUMO-derived peak in the DOS is significantly reduced to $0.14 \mathrm{eV}$ for $\mathrm{K}_{2} \mathrm{PTCDA} / \mathrm{Ag}(111)$, which amounts to nearly a factor of 2 compared to PTCDA/ $\mathrm{Ag}(111)$ (see Supporting Information for more details) (Figure 3c).

Notably, a shift to higher binding energies and a complete filling of the LUMO-derived band is also seen in the experimental UPS data for $\mathrm{K}_{2}$ PTCDA (Figure 3e). A similar observation has in fact been made in UPS studies of PTCDA on the $\operatorname{Ag}(110)$ surface upon potassium deposition in spite of the fundamentally different interface geometry at that metal surface, with $\mathrm{K}$ atoms substituting $\mathrm{Ag}$ atomic rows below the oxygens of the PTCDA molecules. ${ }^{42}$

For $\mathrm{K}_{4} \mathrm{PTCDA} / \mathrm{Ag}(111)$ the trends already observed for $\mathrm{K}_{2}$ PTCDA/ $\mathrm{Ag}(111)$ prevail both in the simulations and in the experiments, with the shift toward higher binding energies being somewhat larger. Concomitantly, the net charge on the PTCDA molecules increases (Table 1). Also the peaks in the PDOS sharpen further, and the intrinsic line width of the LUMO-derived feature is reduced by another factor of 2 to 0.07 $\mathrm{eV}$ (see Supporting Information). At the same time, the splitting between the LUMO+1 and the LUMO+2 increases compared to lower doping stages. The scenario of charge transfer depicted here is additionally validated by the excellent agreement between the experimental and calculated changes of the surface work function with doping (vide infra).

The main conclusion from the above considerations is that at none of the doping stages is a K-induced reduction of the electron density in the PTCDA layer observed, as had been tentatively assumed based on the similar positions of the main peaks in the DR spectra of $\mathrm{K}_{2}$ PTCDA/Ag(111) and PTCDA/ $\mathrm{Au}(111)$ layers (vide supra). Thus, to reconcile the calculated PDOSs and measured UPS data with the results of the DRS measurements, a more in-depth discussion of the optical properties of pristine and doped PTCDA layers is required.

Simulated Optical Properties of Molecules and Adsorbate Layers. Ideally, the simulations of the optical properties should account for the $2 \mathrm{D}$ extended interfaces including the metal substrate. For that, slab-type calculations employing periodic boundary conditions would be required. At the same time excitonic effects need to be accounted for, as they are highly relevant in molecular layers. Indeed, simulations including the necessary many-body effects also for infinitely extended crystalline systems are generally possible. They rely, for example, on solving the Bethe-Salpeter equation (BSE) and have been performed for molecular and polymer crystals. ${ }^{43-46}$ Recently, even implementations of the BSE for adsorbed molecules have been developed, ${ }^{47}$ building, however, on system-specific assumptions that do not apply here (e.g., that the adsorbate's HOMO lies in the band gap of a semiconducting substrate).

Consequently, BSE-type calculations on interfaces as complex as the present one are still beyond reach. Therefore, we resorted to a dual strategy: We first calculated optical properties including excitonic effects for neutral and charged PTCDA molecules in the gas phase using time-dependent density functional theory (TD-DFT). There, we tried to correlate the properties of the excitons with those of the molecular orbitals that dominate their description. Provided that the exciton is primarily represented by the excitation from a specific occupied to a specific unoccupied orbital, the optical properties of the interface can be directly correlated with the evolutions of the calculated densities of states. As a second step, we also simulated excitation spectra based on the independent particle approximation relying on the calculated Kohn-Sham orbitals of the extended interfaces. In these calculations the interaction with the substrate and with dopant atoms is directly considered, but excitonic effects are neglected.

Following that strategy, we report the molecular excitation energies and excited-state properties (obtained using TD-DFT) in Table 2. The corresponding orbital energies are contained in the Supporting Information together with the isodensity plots of the orbitals. Several relevant insights can be gained from those data: (i) The main absorption features of the neutral PTCDA molecule and the dianion are found at essentially the same energies $(2.17 \mathrm{eV}$ vs $2.25 \mathrm{eV})$. This occurs in spite of the fact that they are dominated by different single-particle excitations (the HOMO $\rightarrow$ LUMO transition in the neutral case and a transition from the now doubly occupied LUMO to the LUMO+2 in the dianion). Consequently, the similarity of the transition energies and oscillator strengths for those peaks are a mere coincidence. Therefore, the similarity of the peak positions in the measured optical absorption data of $\mathrm{K}_{2} \mathrm{PTCDA} / \mathrm{Ag}(111)$ and PTCDA/Au(111) discussed above do not necessarily imply that the two films are in the same charge state. (ii) In the dianion there is a weaker peak at $\sim 0.5$ $\mathrm{eV}$ below the main maximum. This peak is dominated by a LUMO to LUMO+1 excitation and can be associated with the lower energy maximum in the measured $\varepsilon_{\text {ads }}^{\prime \prime}$ spectra for Kdoped PTCDA films in Figure 2. Indeed, the oscillator strength associated with this peak is almost an order of magnitude smaller than that of the LUMO to LUMO+2 excitation contributing to the main maximum. Simulations on a cluster consisting of a PTCDA molecule asymmetrically coordinated with two Na atoms yielded similar results (albeit with modified indices for the orbitals and excited states due to the explicit consideration of the dopants). ${ }^{48} \mathrm{We}$ also calculate the most prominent Raman-allowed vibrational mode of the PTCDA dianion, which is at $0.20 \mathrm{eV}$. This is almost exactly the energy by which the vibronic replicas are shifted from the main peaks in the experimental spectra for the dianion on mica and for 
Table 2. TD-DFT-Calculated Properties of the Lowest-Lying Optically Allowed Excited States of PTCDA Molecules at Various Charging Stages ${ }^{a}$

\begin{tabular}{|c|c|c|c|c|c|}
\hline PTCDA & state & $\begin{array}{c}\text { energy } \\
(\mathrm{eV})\end{array}$ & $\begin{array}{l}\text { oscillator } \\
\text { strength }\end{array}$ & $\begin{array}{l}\text { dominating } \\
\text { contributions } \\
\text { including their } \\
\text { weights }{ }^{b}\end{array}$ & $\begin{array}{c}\text { orbital } \\
\text { gap } \\
(\mathrm{eV})\end{array}$ \\
\hline \multirow{3}{*}{$\begin{array}{l}\text { neutral } \\
\text { monoanion }\end{array}$} & $S_{1}$ & 2.17 & 0.48 & $0.71 \mathrm{H} \rightarrow \mathrm{L}$ & 1.50 \\
\hline & $\mathrm{S}_{2}$ & 1.57 & 0.03 & $0.99 \mathrm{~L}_{\alpha} \rightarrow \mathrm{L}+1_{\alpha}$ & 1.42 \\
\hline & $\mathrm{S}_{3}$ & 2.04 & 0.53 & $\begin{array}{l}0.82 \mathrm{~L}_{\alpha} \rightarrow \mathrm{L}+2_{\alpha} \\
0.56 \mathrm{H}_{\beta} \rightarrow \mathrm{L}_{\beta}\end{array}$ & $\begin{array}{l}1.53 \\
1.31\end{array}$ \\
\hline \multirow[t]{2}{*}{ dianion } & $S_{1}$ & 1.82 & 0.07 & $0.70 \mathrm{~L} \rightarrow \mathrm{L}+1$ & 1.41 \\
\hline & $\mathrm{S}_{3}$ & 2.25 & 0.59 & $0.70 \mathrm{~L} \rightarrow \mathrm{L}+2$ & 1.57 \\
\hline
\end{tabular}

${ }^{a}$ As discussed in the main text, $\mathrm{H}$ and $\mathrm{L}$ refer to the orbitals that have the same symmetry as the HOMO and LUMO of the neutral molecule (consequently, L for the dianion refers to the highest doubly occupied orbital), while $\mathrm{L}+1$ and $\mathrm{L}+2$ refer to the orbitals that have the same symmetry as the second and third unoccupied orbital. Importantly, the orbitals with a certain label have essentially the same shapes at different doping levels. The subscripts $\alpha$ and $\beta$ refer to the two spin manifolds. There is no significant change in shape and, more importantly, no change in their order. ${ }^{b}$ The observation that the $S_{1}$ and $S_{3}$ coefficients in the description of the excitations in the neutral and dianion case appear to be $1 / \sqrt{2}$ smaller than $S_{2}$ for the anion is just a consequence of the fact that for a spin-restricted calculation a normalization factor of $1 / \sqrt{2}$ is included in the description of the singlet state in the Gaussian output. This is not the case for the spinunrestricted calculation of the anion. In the characterization of the excited states only transitions with weights exceeding 0.13 are included.

$\mathrm{K}_{4} \mathrm{PTCDA} / \mathrm{Ag}(111)$ (see Figure 2). (iii) In the monoanion, the most prominent optical excitation (again dominated by a LUMO $\rightarrow$ LUMO+2 transition) is somewhat red-shifted compared to the neutral and dianion cases. This is reminiscent of the situation of PTCDA on $\operatorname{Ag}(111)$ in the absence of the potassium atoms. The calculated shift is, however, smaller than in the experiment. Some deviations between simulations and experiments are not surprising though, bearing in mind that upon adsorption on $\operatorname{Ag}(111)$ the PTCDA LUMOs are more than half-filled (i.e., the actual situation goes beyond monoanion formation). Moreover, on the surface the filling of the LUMO is partially compensated by a back-transfer of charges from the carboxylic oxygens (vide supra). ${ }^{49}$ None of these effects are captured in the calculations of the isolated monoanion.

Interestingly, all relevant optically allowed excited states in the neutral PTCDA molecule and the dianion (i.e., the systems most relevant for the present study) are dominated by individual single-particle excitations. Therefore, the properties of the excited states can be immediately associated with those of specific orbitals. The observation that the absolute values of the Kohn-Sham orbital gaps are much smaller than the excited-state energies can be traced back to the self-interaction error $^{50}$ and lack of the derivative discontinuity in (semi)local functionals and has no bearing on the qualitative conclusions drawn here. What is important are the observed trends. For example, the HOMO $\rightarrow$ LUMO gap in the neutral PTCDA molecule (also reported in Table 2) is only $0.07 \mathrm{eV}$ smaller than the LUMO $\rightarrow$ LUMO +2 gap in the dianion, which is fully consistent with an energetic difference of $0.08 \mathrm{eV}$ between the respective states $\left(S_{1}\right.$ for the neutral molecule and $S_{3}$ for the dianion).
To link these results to the calculated densities of states discussed in the previous section, it is worth pointing out that several trends observed in the band-structure calculations of the interfaces (Figure 3) are directly reflected in the shifts of molecular orbital energies (Table S1) (recalling that PTCDA is negatively charged by $\mathrm{Ag}(111)$ even without $\mathrm{K})$ : The HOMO $\rightarrow$ LUMO gap is strongly reduced upon charging both in the interface simulations and in the molecular calculations. Conversely, the LUMO $+1 \rightarrow \mathrm{LUMO}+2$ splitting increases upon $\mathrm{K}$ doping on the surface and likewise upon charging the individual molecules ( $c f$. ., Figure 3 and Table S1). These correlations indicate that trends derived from single-particle densities of states of extended interfaces can be directly correlated with excitation processes within the PTCDA adsorbate layer. Consequently, it is also useful to calculate optical spectra (within the independent particle approximation) from the bands of the extended interfaces taking the hybridization of the molecular and substrate states into account.

The corresponding spectra obtained within the independent particle random phase approximation (IP-RPA) are shown in

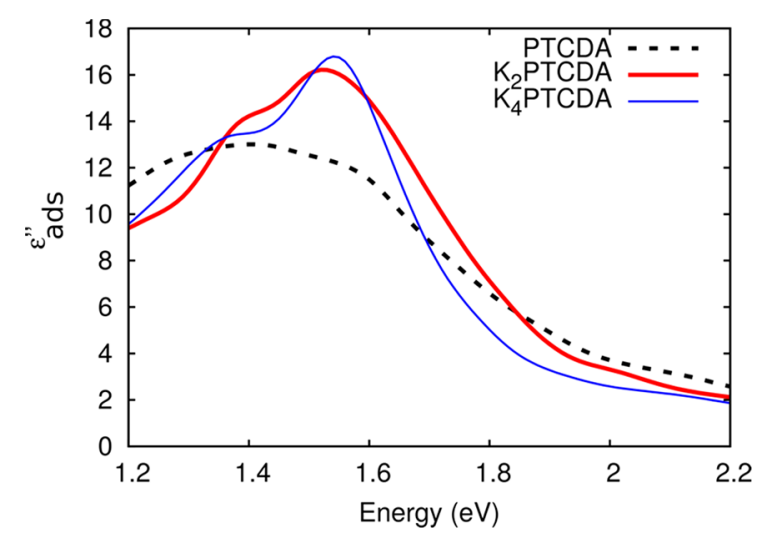

Figure 4. Imaginary part of the dielectric function for extended PTCDA/Ag(111), $K_{2}$ PTCDA/Ag(111), and $K_{4} P T C D A / \operatorname{Ag}(111)$ interfaces calculated from the band structures employing the independent particle random phase approximation (IP-RPA). A Lorentzian broadening of $0.14 \mathrm{eV}$ (FWHM) is applied to the plotted data.

Figure 4. The displayed dielectric function of the adsorbed monolayer is computed as ${ }^{51}$

$$
\varepsilon_{\mathrm{ads}}^{\prime \prime}=\Delta \varepsilon_{\mathrm{sc}}^{\prime \prime} d_{\mathrm{sc}} / d_{\mathrm{ML}}
$$

where $\Delta \varepsilon_{\mathrm{sc}}^{\prime \prime}=\varepsilon_{\mathrm{sc}, \mathrm{K}_{x} \mathrm{PTCDA} / \mathrm{Ag}(111)}^{\prime \prime}-\varepsilon_{\mathrm{sc}, \mathrm{Ag}(111)}^{\prime \prime}$ is the change in the dielectric function of the supercell upon adding $\mathrm{K}_{x} \mathrm{PTCDA}$ to the Ag surface; $d_{\mathrm{sc}}$ is the height of the supercell used in simulations in the direction perpendicular to the surface (slab thickness + vacuum region), and $d_{\mathrm{ML}}$ is the thickness of the adsorbed monolayer (estimated to be $3.25 \AA$ on the basis of the bulk density of PTCDA ${ }^{52}$ ). One observes that the energies of the maxima in Figure 4 correspond to energetic splittings of the DOS peaks in Figure 3 for exactly those orbitals that most strongly contribute to the description of the excited states (cf., Table 2). For example, the maximum for the doped films at about $1.55 \mathrm{eV}$ corresponds to the energy difference of the LUMO- and LUMO+2-derived bands in the corresponding DOS, which also amounts to $1.55 \mathrm{eV}$. This confirms the above interpretation of the spectra and enables us to address the impact of hybridization with the substrate: In the calculations, 

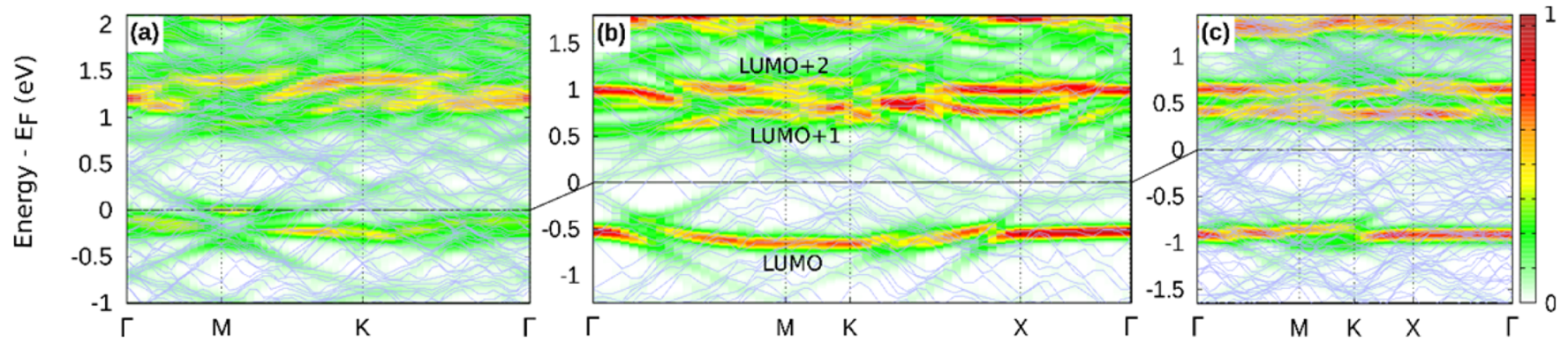

Figure 5. Band structure for (a) PTCDA/Ag(111), (b) $\mathrm{K}_{2} \mathrm{PTCDA} / \operatorname{Ag}(111)$, and (c) $\mathrm{K}_{4} \mathrm{PTCDA} / \operatorname{Ag}(111)$. The $k$-resolved DOS (KDOS( $k$, E)) (for details see main text and Methods section) projected onto the molecular region is superimposed as an intensity plot to show the contributions from PTCDA molecular orbitals. Note the different energy ranges, to follow the energy shift of the PTCDA orbitals with K doping (the total width of the displayed energy range is the same in all panels). The color scale for the KDOS is normalized to the maximum value for a nondegenerate state fully localized on the molecule (see Methods section).

the spectrum of PTCDA/ $\operatorname{Ag}(111)$ (black curve in Figure 4) is characterized by a peak at $1.4 \mathrm{eV}(1.75 \mathrm{eV}$ in the experiments, Figure 2a). This peak narrows and shifts to higher energy for $\mathrm{K}_{2}$ PTCDA/Ag(111) (red curve in Figure 4). Both trends are consistent with the experimental observations (see Figure 2a) and prevail for $\mathrm{K}_{4} \mathrm{PTCDA} / \mathrm{Ag}(111)$ with a slightly larger shift of the main feature (see blue curve in Figure 4). Also the experimentally observed splitting into a weaker low-energy feature and a stronger high-energy feature for $\mathrm{K}_{2} \mathrm{PTCDA} /$ $\mathrm{Ag}(111)$ and $\mathrm{K}_{4} \mathrm{PTCDA} / \mathrm{Ag}(111)$ is reproduced in the simulations. In passing we note that a fully quantitative agreement between theory and experiments is not expected within the independent particle approximation, and also the vibronic fine-structure observed in the experiments is not contained in simulations focusing on electronic excitations.

The decreasing line width of the spectral features after $\mathrm{K}$ doping can be directly traced back to the decreasing line widths of the relevant DOS peaks discussed in the previous section. The most relevant bands in this context are the ones derived from the molecular LUMO and LUMO+2 for the main peak and, additionally, the LUMO+1-derived band for the lowenergy tail of the main feature.

These considerations allow us to unambiguously associate the DR spectra at various K-doping stages with specific charging states of the adsorbate layer. Particularly, they show that the similar peak positions for $\mathrm{K}_{2} \mathrm{PTCDA} / \mathrm{Ag}(111)$ and $\mathrm{PTCDA} / \mathrm{Au}(111)$ are a coincidence rather than an indication for a reduction of the charge of the PTCDA layer due to $\mathrm{K}$ deposition. The reduced width of the spectral features upon $\mathrm{K}$ doping is an indication for a reduced coupling to the substrate, ${ }^{28}$ an aspect that shall be addressed more thoroughly in the following sections.

Coupling between the PTCDA Layer and the Ag(111) Substrate at Various Stages of K-Doping: Band Structures and Adsorption Heights. Given that optical transitions do not mix states with different $k$-vectors, studying the actual band structures of the interfaces provides insight beyond merely considering the densities of states. The calculated bands for PTCDA/Ag(111), K 2 PTCDA/Ag(111), and $\mathrm{K}_{4} \mathrm{PTCDA} / \mathrm{Ag}(111)$ are shown as thin lines in Figure 5. Superimposed is a $k$-resolved density of states projected onto atom-centered functions of the molecules $(\operatorname{KDOS}(k, E)),{ }^{53,54}$ in anaology to the evaluation of Löwdin charges. ${ }^{55}$ That quantity is highest for $(k, E)$ values corresponding to states localized in the molecular regions; that is, it allows us to identify molecular contributions to the actual band structure. The
KDOS could also be plotted by coloring each point in the band structure depending on the square of the wave function amplitude in the molecular region of the adsorbate layer. ${ }^{56,57}$ This would, however, generate a somewhat involved picture considering the fairly large number of bands due to using a slab of a finite thickness to represent the metal substrate. This complication can be avoided by representing the KDOS in Figure 5 as a color map adopting a Gaussian broadening for each state using a width of $0.11 \mathrm{eV}$, i.e., the same broadening that was applied when plotting the DOS in Figure 3 (for details see Methods section).

For PTCDA/ $\operatorname{Ag}(111)$ one observes a massive energetic broadening of the PTCDA-derived LUMO (somewhat below $\left.E_{\mathrm{F}}\right), \mathrm{LUMO}+1$, and LUMO+2 states. This is indicative of the strong hybridization with substrate states at virtually all $k$-points of the surface Brillouin zone. The hybridization is superimposed with a significant dispersion of the main features (red and yellow regions) of the bands, with the combination of both effects giving rise to the large width of the DOS features. For $\mathrm{K}_{2} \mathrm{PTCDA}$, the dominant KDOS features are much more clearly resolved in energy (see the increase in red regions in panel (b) compared to panel (a)). Note also the reduced energetic broadening indicating a reduced hybridization with the substrate. In $\mathrm{K}_{4} \mathrm{PTCDA} / \mathrm{Ag}(111)$, the molecule-derived bands become essentially flat, with the effect being most pronounced for the LUMO+1- and LUMO+2-derived states. This explains why in this system especially the LUMO+1- and LUMO+2-derived DOS peaks are well resolved and clearly separated (see also Figure 3). All these results confirm the reduced interaction between PTCDA and Ag due to K doping.

A difference in molecule-metal interaction should also be reflected in the vertical adsorption geometry of the adsorbate layer and, particularly, in the adsorption heights of the individual atomic species of the $\mathrm{K}_{2} \mathrm{PTCDA}$ structure. The latter can be determined by NIXSW experiments with very high precision and chemical sensitivity. ${ }^{58,59}$ Details of the NIXSW experiments and the data analysis are discussed in the Methods section and the Supporting Information. The key results are illustrated in a structural model of the vertical adsorption geometry in Figure 6 together with the respective calculated values. All experimentally and theoretically determined adsorption heights for $\mathrm{K}_{2} \mathrm{PTCDA}, \mathrm{K}_{4} \mathrm{PTCDA}$, and literature data for PTCDA $/ \operatorname{Ag}(111)^{60,61}$ are summarized in Table 3.

Before discussing the resulting model in detail, it is worthwhile mentioning that measured and calculated heights match well. When comparing the numbers, a general 


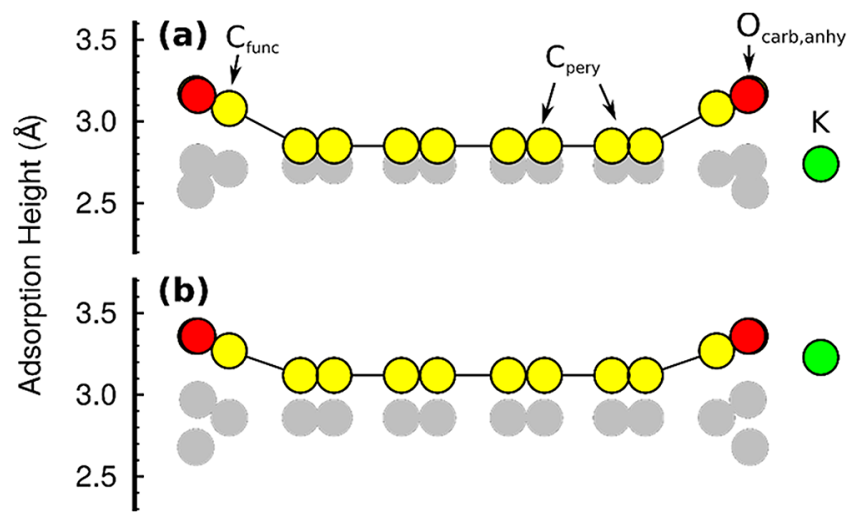

Figure 6. Adsorption geometry and vertical adsorption distances of PTCDA and potassium (see $K$ in green) as determined for $\mathrm{K}_{2}$ PTCDA $/ \mathrm{Ag}(111)$ by means of (a) DFT calculations and (b) NIXSW experiments. Gray spheres represent pristine PTCDA/ $\operatorname{Ag}(111)$ (our calculations in (a) and experimental data from ref 61 in (b)). The adsorption heights of the hydrogen atoms (not shown) were not measured. All distances are reported with respect to the (hypothetical) unrelaxed $\operatorname{Ag}(111)$ surface.

observation is that the calculated adsorption heights are consistently slightly smaller than the experimental ones. We attribute this at least in part to an inaccurate description of the vertical relaxation of the topmost $\mathrm{Ag}$ layer, which for $\mathrm{K}_{2} \mathrm{PTCDA} / \mathrm{Ag}(111)$ and $\mathrm{K}_{4} \mathrm{PTCDA} / \mathrm{Ag}(111)$ also arise from the modifications we had to make to the lattice constants to reconcile the point-on-line growth with periodic boundary conditions (see ref 19 and the Methods section). This Ag relaxation is indeed relevant here, as the true interaction is determined by the actual interatomic distances, while the quantities measured in NIXSW are the distances relative to the lattice planes of the Bragg reflection used, i.e., effectively relative to the (hypothetical) unrelaxed $\mathrm{Ag}(111)$ surface. An incorrectly modeled surface relaxation will, therefore, cause minor deviations between measured and calculated heights. Only for the $\mathrm{K}$ atoms do the deviations appear too large to arise from such effects. To test to what extent the very high experimental $\mathrm{K}$ position is a consequence of varying $\mathrm{K}$-adsorption positions arising from point-on-line growth, we compared $\mathrm{K}$ atoms adsorbed on top and in hollow positions as the two limiting cases. There we, however, found differences in adsorption heights of only $0.02 \AA$ A Another reason for the deviations could be that the adsorption heights measured for $\mathrm{K}_{2} \mathrm{PTCDA}$ might be slightly influenced by a small amount of coexisting $\mathrm{K}_{4}$ PTCDA (see Supporting Information for more details) in such a way that the obtained values are systematically too large (cf. Table 3).

Independent of the above-mentioned deviations, the trends in the main geometrical changes upon $\mathrm{K}$ doping are consistent for simulations and experiments: (i) $\mathrm{K}$ deposition clearly increases the adsorption height of the perylene core of PTCDA above the Ag substrate, which again suggests a weakening of the coupling between the substrate and the adsorbate layer. (ii) While for PTCDA/Ag(111) especially the carboxylic oxygens bend toward the surface indicative of an additional bonding channel to the Ag substrate, upon $\mathrm{K}$ doping they are found at positions significantly above the perylene carbons. Interestingly, the anhydride oxygens also bend away from the surface upon $\mathrm{K}$ doping such that they come to lie above the $\mathrm{K}$ atoms. Note that the adsorption heights of the oxygen atoms are quantitatively the same for both oxygen species, but the differences in adsorption heights between $\mathrm{O}_{\text {anhy }}$ and $\mathrm{O}_{\text {carb }}$ are significantly smaller than in the case of PTCDA/ $\operatorname{Ag}(111)$. The reasons for the upward bending will become apparent when analyzing the interfacial charge rearrangements (vide infra). Upon increasing the $\mathrm{K}$ concentration (i.e., for the $\mathrm{K}_{4} \mathrm{PTCDA} / \mathrm{Ag}(111)$ system), all K-induced geometrical changes are enhanced in the calculations (no experiments are available for that doping stage).

In passing we note that the rather strong lifting of the perylene core away from the Ag surface upon $\mathrm{K}$ doping is in sharp contrast to the situation observed upon exposing the PTCDA $/ \operatorname{Ag}(110)$ interface to $\mathrm{K}$ atoms, where the measured adsorption height increases by only $0.05 \AA{ }^{42}$ We attribute that difference to a significantly altered bonding geometry, as on $\mathrm{Ag}(110)$ the $\mathrm{K}$ atoms lie below the PTCDA molecules replacing $\mathrm{Ag}$ atoms in the topmost surface layer, while in the present case they lie above the top Ag layer. ${ }^{19}$ The latter results in a distinct upward bending of the carboxylic oxygens so that they lie 0.24 $\AA(0.31 \AA)$ above the perylene core in the NIXSW experiments (DFT calculations). Importantly, the decoupling of the PTCDA molecules from the substrate upon $\mathrm{K}$ deposition is not a consequence of any tilting of the molecule, as has been observed when subjecting a $6 \mathrm{P}$ monolayer on $\mathrm{Cu}(110)$ to $\mathrm{Cs}$ doping. ${ }^{21}$ This can be inferred from both the STM images ${ }^{19}$

Table 3. Calculated (Regular) and Measured (Bold) Average Adsorption Heights for Different Chemical Species in PTCDA/ $\operatorname{Ag}(111), K_{2}$ PTCDA $/ \operatorname{Ag}(111)$, and $K_{4}$ PTCDA $/ \operatorname{Ag}(111)^{a}$

\begin{tabular}{|c|c|c|c|c|c|c|}
\hline \multicolumn{2}{|c|}{ system } & $\mathrm{C}_{\text {pery }}(\AA)$ & $\mathrm{C}_{\text {func }}(\AA)$ & $\mathrm{O}_{\text {anhy }}(\AA)$ & $\mathrm{O}_{\text {carb }}(\AA)$ & $\mathrm{K}(\AA)$ \\
\hline \multirow[t]{2}{*}{ PTCDA/Ag(111) } & theory $^{b}$ & $2.73(2.82)$ & $2.71(2.80)$ & $2.75(2.84)$ & $2.58(2.66)$ & \\
\hline & experiment ${ }^{c}$ & \multicolumn{2}{|c|}{$2.86( \pm 0.01)$} & $2.98( \pm 0.08)$ & $2.66( \pm 0.03)$ & \\
\hline \multirow[t]{2}{*}{$\mathrm{K}_{2} \mathrm{PTCDA} / \mathrm{Ag}(111)$} & theory & $2.85(2.98)$ & $3.08(3.21)$ & $3.16(3.29)$ & $3.17(3.30)$ & $2.74(2.87)$ \\
\hline & experiment & $3.12( \pm 0.02)$ & $3.26( \pm 0.07)$ & $3.36( \pm 0.05)$ & $3.36( \pm 0.07)$ & $3.23( \pm 0.03)$ \\
\hline $\mathrm{K}_{4} \mathrm{PTCDA} / \mathrm{Ag}(111)$ & theory & $3.04(3.10)$ & $3.34(3.41)$ & $3.43(3.50)$ & $3.45(3.51)$ & $2.72(2.79)$ \\
\hline
\end{tabular}

${ }^{a}$ All values are given relative to the (hypothetical) unrelaxed $\mathrm{Ag}(111)$ surface, as this is the quantity measured in X-ray standing wave experiments. Additionally, in parentheses we report the calculated distances relative to the relaxed top Ag layer. The values given in parentheses along with the measured adsorption heights are the experimental uncertainties. $C_{\text {pery }}$ denotes the $\mathrm{C}$ atoms in the perylene core, $\mathrm{C}_{\text {func }}$ are those attached to the $\mathrm{O}$ atoms, $\mathrm{O}_{\text {anhy }}$ denotes the anhydride oxygens, $\mathrm{O}_{\text {carb }}$ are the carboxylic oxygens, and $\mathrm{K}$ are the potassium atoms. The adsorption heights of the hydrogen atoms were not measured. ${ }^{b}$ These distances are ca. $0.1 \AA$ smaller than the ones reported in ref 62 . This is mostly due to the fact that we here considered a five-layer $\mathrm{Ag}$ slab in which the two top layers were relaxed, while in ref 62 only three layers were considered, where just one was allowed to change its position during geometry relaxation. When repeating our geometry optimizations with that reduced-size model system, deviations between our calculations and those in ref 62 are at the most $0.04 \AA$; thus, the main reduction can be explained by a modified relaxation of the Ag surface. ${ }^{c}$ Taken from ref 61 ; see also ref 60 . 

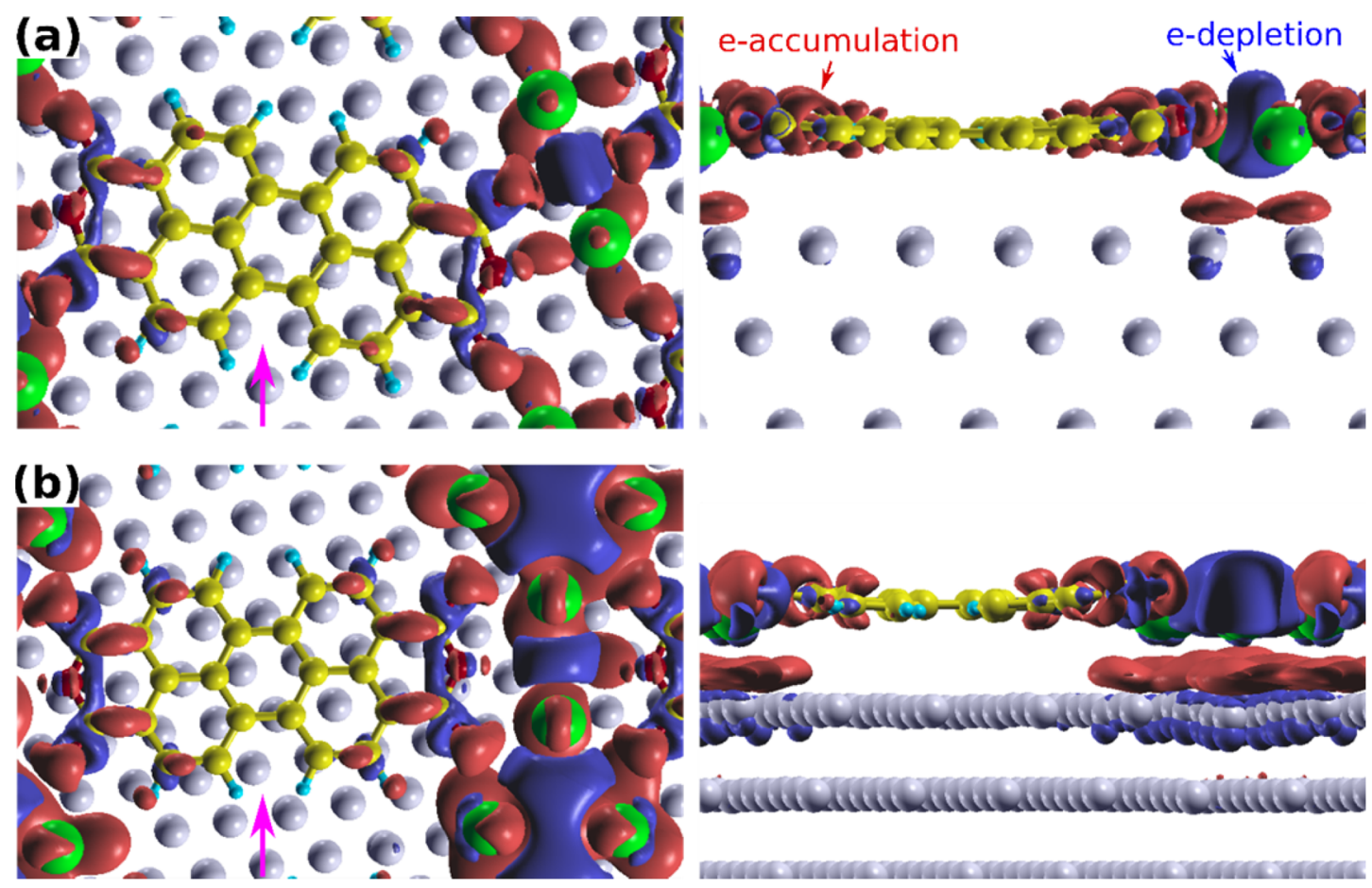

Figure 7. Top and side views (as seen in the direction indicated by the magenta arrow in the left panels) for the charge rearrangements occurring upon $\mathrm{K}$ doping in the $\mathrm{K}_{x} \mathrm{PTCDA} / \operatorname{Ag}(111)$ systems. (a) Situation for $\mathrm{K}_{2} \mathrm{PTCDA} / \operatorname{Ag}(111)\left(\right.$ i.e., $\Delta \rho=\rho\left(\mathrm{K}_{2} \mathrm{PTCDA} / \operatorname{Ag}(111)\right)-$ $\rho(\mathrm{PTCDA} / \operatorname{Ag}(111))-\rho\left(\mathrm{K}_{2}\right)$; (b) equivalent plots for $\mathrm{K}_{4} \mathrm{PTCDA} / \operatorname{Ag}(111)$ (i.e., $\Delta \rho=\rho\left(\mathrm{K}_{4} \mathrm{PTCDA} / \operatorname{Ag}(111)\right)-\rho(\mathrm{PTCDA} / \operatorname{Ag}(111))-$ $\rho\left(\mathrm{K}_{4}\right)$ ). Red regions show electron accumulation, and blue regions show electron depletion (isovalue $=0.002 \mathrm{e} / \mathrm{Bohr}^{3}$ ). The color code of $\mathrm{C}$, $\mathrm{O}$, and $\mathrm{H}$ atoms corresponds to that in Figure 1, while Ag atoms are depicted in gray here.

and the high coherent fractions of the $\mathrm{C}_{\text {pery }}$ atoms (see Supporting Information) in the NIXSW experiments.

To determine to what extent the reduced widths of the DOS features in $\mathrm{K}_{2} \mathrm{PTCDA} / \mathrm{Ag}(111)$ compared to PTCDA/ $\mathrm{Ag}(111)$ are a consequence of geometric deformations and to what extent they are caused by the electronic interactions with the $\mathrm{K}$ atoms, we studied a model structure with all atoms in the same coordinates as in $\mathrm{K}_{2} \mathrm{PTCDA} / \mathrm{Ag}(111)$, but removed $\mathrm{K}$. The resulting band structure and DOS, as well as additional intermediate models, are presented in the Supporting Information and testify that $\mathrm{K}$ atoms act both structurally by raising the molecule and electronically by weakening PTCDA$\mathrm{Ag}$ and PTCDA-PTCDA interactions.

Charge Rearrangements. To better understand the interaction of the PTCDA layer with the $\mathrm{K}$ atoms, it is useful to analyze charge rearrangements, $\Delta \rho$, upon $\mathrm{K}$ doping. Here it should be noted that the purpose of the following gedanken experiment is not to mimic the actual growth process, but rather to analyze the way charges are redistributed due to the doping of $\mathrm{K}$ atoms into the PTCDA film on Ag. $\Delta \rho$ is given by the difference of the charge densities of the interacting system and the subsystems (all in the geometry of $\mathrm{K}_{x} \mathrm{PTCDA} /$ $\operatorname{Ag}(111))$ :

$$
\begin{aligned}
\Delta \rho= & \rho\left(\mathrm{K}_{x} \mathrm{PTCDA} / \operatorname{Ag}(111)\right)-\rho(\mathrm{PTCDA} / \operatorname{Ag}(111)) \\
& -\rho\left(\mathrm{K}_{x}\right)
\end{aligned}
$$

Here, $\rho\left(\mathrm{K}_{x} \mathrm{PTCDA} / \operatorname{Ag}(111)\right), \rho(\mathrm{PTCDA} / \operatorname{Ag}(111))$, and $\rho\left(\mathrm{K}_{x}\right)$ denote the charge densities of the K-doped PTCDA layer on $\mathrm{Ag}(111)$, the adsorbed system upon removing the $\mathrm{K}$ atoms, and the removed $\mathrm{K}$ atoms, respectively. An alternative scenario, where we first consider the formation of a hypothetical free-standing $\mathrm{K}_{x}$ PTCDA layer, which eventually adsorbs on the $\operatorname{Ag}(111)$ substrate, is discussed in the Supporting Information.

The charge rearrangements for the formation of the PTCDA/Ag(111) interface (with a herringbone arrangement of the molecules) have already been discussed extensively in the literature (e.g., in ref 49). There one primarily observes an electron transfer into the $\pi$-electron system of the PTCDA layer accompanied by an electron density reduction above the metal and in the $\sigma$-electron system, with the latter effect strongest in the region of the carboxylic oxygens. This is indicative of the charge back-donation process described earlier. A similar situation is obtained when arranging the molecules on $\mathrm{Ag}(111)$ in the geometry they finally adapt in the $\mathrm{K}_{2}$ PTCDA/ $\operatorname{Ag}(111)$ layer (see Supporting Information).

When adding the $\mathrm{K}$ atoms ( $c f .$, Figure $7 \mathrm{a}$ ) to the adsorbed PTCDA layer, one observes the additional donation of electrons into the $\pi$-system of the PTCDA molecules especially in the molecular regions close to the $\mathrm{K}$ atoms. This is consistent with a further filling of the LUMO upon $\mathrm{K}$ doping. Additionally, one sees electron density accumulation above the topmost Ag layer especially directly below the $\mathrm{K}$ atoms. An interesting observation is that the most significant electron density depletion is not centered at the $\mathrm{K}$ atoms but localized in the region between them. This is also observed when potassium atoms are adsorbed on the bare $\operatorname{Ag}(111)$ surface in the absence of the PTCDA layer (see Supporting Information). An additional feature is the accumulation of electron density on top of the $\mathrm{K}$ atoms, which is a common observation for $\mathrm{K}$ and heavier alkalis adsorbed on metals. ${ }^{63}$

Of particular interest are the charge rearrangements in the immediate vicinity of the oxygen atoms: There is electron density accumulation between both the carboxylic and 
anhydride oxygens and the $\mathrm{K}$ atoms. Concomitantly, electron density is depleted between the oxygens and the neighboring carbon atoms. This is indicative of a modification of the bonding situation of the oxygens with a weakening of the bonds to the backbone of the PTCDA molecules and an interaction with the $\mathrm{K}$ atoms that goes clearly beyond a mere charge transfer. This observation reinforces the above conclusion that the direct chemical interaction between the anhydride groups of the PTCDA molecules and the $\mathrm{K}$ atoms plays a crucial role for the reduced coupling between PTCDA and $\operatorname{Ag}(111)$ upon $\mathrm{K}$ doping. Similar trends, albeit with more massive charge rearrangements, are also seen for the $\mathrm{K}_{4} \mathrm{PTCDA}$ system (see Figure $7 b$ ).

As a final remark, we briefly mention work function changes, as a direct consequence of the charge rearrangements (especially those perpendicular to the metal surface). They are significant, as shown in Table 4 with $\mathrm{K}$-doping triggering a

Table 4. Comparison between Experimentally Measured and Simulated Work-Function Changes with Respect to the Bare Substrate, $\Delta \Phi^{\operatorname{Ag}(111)}$, and Relative to Undoped PTCDA/ $\operatorname{Ag}(111), \Delta \Phi^{\text {PTCDA } a}$

\begin{tabular}{|c|c|c|c|c|}
\hline \multirow[b]{2}{*}{$\begin{array}{c}\mathrm{K}_{x} \mathrm{PTCDA} / \\
\mathrm{Ag}(111)\end{array}$} & \multirow{2}{*}{$\begin{array}{c}\begin{array}{c}\Delta \Phi^{\mathrm{Ag}(111)} \\
(\mathrm{eV})\end{array} \\
\text { simulated }\end{array}$} & \multicolumn{3}{|c|}{$\Delta \Phi^{\mathrm{PTCDA}}(\mathrm{eV})$} \\
\hline & & simulated & $\begin{array}{c}\text { experimental } \\
(0.7 \mathrm{ML})\end{array}$ & $\begin{array}{c}\text { experimental } \\
\text { (extrapolated to } 1.0 \\
\text { ML) }\end{array}$ \\
\hline$x=0$ & +0.16 & & & \\
\hline$x=2$ & -0.64 & -0.80 & -0.6 & -0.9 \\
\hline$x=4$ & -1.42 & -1.58 & -1.1 & -1.6 \\
\hline
\end{tabular}

${ }^{a} \mathrm{~A}$ complication in the experiments is that the surface coverage is incomplete $(d=0.7 \mathrm{ML})$. In order to allow for a better comparison to the simulated values, the measured work-function changes were, thus, extrapolated to monolayer coverage (by dividing by 0.7 ). The overall agreement is excellent, although a significant amount of $\mathrm{K}$ not interacting with PTCDA may exist on the samples particularly in the $\mathrm{K}_{4} \mathrm{PTCDA}$ film.

work-function change of up to $-1.6 \mathrm{eV}$. Notably, also for this observable, we obtain an excellent agreement between experiments and simulations, which supports the validity of the calculated charge rearrangements discussed in the preceding paragraphs of this section.

\section{CONCLUSIONS}

The combination of the results from various complementary experimental and theoretical investigations shows that for the prototypical PTCDA/Ag(111) interface extensive geometrical reorderings occur upon potassium doping, which in turn result in massive modifications of the electronic and optical properties of the interface. In particular, K-deposition increases the net electronic charge of the PTCDA molecules, which results in a transition from a metallic to a semiconducting adsorbate layer. This behavior is in sharp contrast to the impact of (degenerate) doping in conventional semiconductors, where doping generates a highly conducting state.

Moreover, $\mathrm{K}$ deposition reduces the electronic coupling between the PTCDA layer and the Ag substrate. In the simulations, this is manifested in a reduction of the intrinsic widths of the DOS features associated with the adsorbate layer and a decreased dispersion and hybridization of the adsorbateprojected bands. In the experiments it gives rise to sharper spectral features upon increasing the $\mathrm{K}$ content in conjunction with an increasing adsorption distance of the perylene core and a pronounced upward-bending of the carboxylic $\mathrm{O}$ atoms. This implies that the strength of the electronic coupling between the molecules and the substrate can be tuned by the potassium dopants. Notably, in spite of the new $\mathrm{K}-\mathrm{O}$ bonding channels and the associated massive charge rearrangements, we do not observe a stronger dispersion of the bands for $\mathbf{k}$-vectors parallel to the substrate surface.

On more technical grounds we find that the observation that the main peak of the DRS spectrum of $\mathrm{K}_{2} \mathrm{PTCDA} / \mathrm{Ag}(111)$ is found at essentially the same position as the dominant peak in the spectrum of PTCDA/Au(111) is not a consequence of an actual chemical similarity between the two situations. Rather it is a mere coincidence arising from the specific positions of the unoccupied orbitals of PTCDA. This shows that such comparisons can be very misleading for complex surfaces and that, for situations as involved as the present one, only the combination of a manifold of complementary experimental techniques with state-of-the-art quantum-mechanical calculations allows a consistent, in-depth atomistic understanding of the actual situation. This understanding is, however, a vital requirement for a future rational design of complex metalorganic interfaces.

\section{METHODS}

Sample Preparation. The $\operatorname{Ag}(111)$ substrate was prepared by repeated $\mathrm{Ar}^{+}$-sputtering and annealing cycles according to ref 64 . Each substrate preparation was checked by means of low-energy electron diffraction (LEED) prior to film deposition. The molecules were deposited under ultra-high-vacuum conditions (base pressure $10^{-10}$ mbar) from effusion cells with temperature-stabilized $\left(\begin{array}{ll} \pm 1 & \mathrm{~K}\end{array}\right)$ crucibles. The deposition rates were on the order of $0.1 \mathrm{ML}$ per minute. The starting point of each doping experiment was a submonolayer of PTCDA on $\mathrm{Ag}(111)$ with a coverage of 0.6 to 0.7 ML. Potassium was subsequently deposited from a commercially available dispenser source (SAES Getters and Alvatec), while the substrate was kept at room temperature. The evolution of the phases was followed by in situ optical spectroscopy and by distortioncorrected LEED experiments to ensure that the above-mentioned PTCDA/Ag(111), $\mathrm{K}_{2} \mathrm{PTCDA} / \mathrm{Ag}(111)$, and $\mathrm{K}_{4} \mathrm{PTCDA} / \operatorname{Ag}(111)$ phases are the only (ordered) ones that occur on the sample. ${ }^{19}$

In Situ Optical Spectroscopy. From the DRS raw data, shown in the Supporting Information, the imaginary part of the dielectric function of the adsorbate layer $\varepsilon_{\text {ads }}^{\prime \prime}$ was extracted by applying the following expression: ${ }^{28}$

$$
\varepsilon_{\mathrm{ads}}^{\prime \prime} \approx-\frac{R(d)-R(0)}{R(0)} \frac{\lambda}{8 \pi d} \frac{\left(1-\varepsilon_{\mathrm{sub}}^{\prime}\right)^{2}+\left(\varepsilon_{\mathrm{sub}}^{\prime \prime}\right)^{2}}{1-\varepsilon_{\mathrm{sub}}^{\prime}}
$$

Here, $\varepsilon_{\text {sub }}^{\prime}$ and $\varepsilon_{\text {sub }}^{\prime \prime}$ are the real and imaginary parts of the dielectric function of the bulk substrate. This approximation is valid for film thicknesses $d$ much smaller than the photon wavelengths $\lambda$ and under the condition that $\left|\varepsilon_{\text {sub }}^{\prime \prime}\right| \ll\left|1-\varepsilon_{\text {sub }}^{\prime}\right|$ holds (which is fulfilled for silver in the wavelength range considered here). ${ }^{65} R(0)$ denotes the reference reflectance of the pristine $\operatorname{Ag}(111)$ substrate, while $R(d)$ stands for the reflectance measured as a function of the effective PTCDA film thickness $d$. For the subsequent K doping $R$ and hence $\varepsilon^{\prime \prime}$ change as a function of the number of adsorbed $\mathrm{K}$ atoms, which itself is assumed to scale linearly with deposition time. For DRS, an actively cooled $100 \mathrm{~W}$ halogen lamp (Osram Xenophot) was operated with a stabilized power supply (Voltcraft HPS-11530). The reflected light was spectrally analyzed by means of a monochromator (Acton Research SpectraPro SP2356) with a charge-coupled device attached (Princeton Instruments Spec-10 100BR, liquid-nitrogen-cooled CCD).

Photoelectron Spectroscopy Experiments. XPS and UPS measurements were performed in Jena using a FOCUS 500 monochromatized $\mathrm{X}$-ray ( $\mathrm{Al} \mathrm{K} \alpha$ emission) and a monochromatized 
UV light source UVLS (He I $\alpha$ emission) combined with a toroidalmirror monochromator TMM 304. The photoelectrons were analyzed using a PHOIBOS 150 hemispherical energy analyzer with a 3D-DLD detector attached. All parts were supplied by SPECS. The acceptance angle of the detector is $\pm 7^{\circ}$. The Fermi edge region has been fitted by a convolution of the Fermi-Dirac function with a Gaussian representing the instrumental resolution, which was thereby estimated to be $\sim 50 \mathrm{meV}$. For the determination of work functions, secondary electron cut-offs were measured with the sample biased at $c a .-9 \mathrm{~V}$. The UP spectra were recorded at normal emission. The PTCDA on $\mathrm{Ag}(111)$ samples were transferred to the photoelectron spectroscopy setup prior to the potassium deposition using an ultra-high-vacuum shuttle with a base pressure of $10^{-10}$ mbar.

Normal Incidence X-ray Standing Wave Experiments. NIXSW experiments utilize the standing wave that forms by the interference of an incident X-ray beam with an outgoing beam that is Bragg diffracted by a single crystal (the substrate). Scanning the photon energy through the Bragg condition shifts the nodes and antinodes of the standing wave in the direction perpendicular to the Bragg planes (in our experiment we use the (111) Bragg planes, which are aligned with the sample surface). This changes the amplitude of the X-ray field at the positions of a specific atomic species in a way that is characteristic for the (vertical) position of that atomic species relative to the nearest Bragg plane. These intensity profiles are parametrized by the coherent fraction $F^{\mathrm{H}}$ and coherent position $P^{\mathrm{H}}$ through

$$
Y\left(E_{\mathrm{ph}}\right)=1+R\left(E_{\mathrm{ph}}\right)+2 \sqrt{R\left(E_{\mathrm{ph}}\right)} F^{\mathrm{H}} \cos \left(\nu\left(E_{\mathrm{ph}}\right)-2 \pi P^{\mathrm{H}}\right)
$$

The so-called yield, $Y$, is the intensity of the standing wave field at the species of interest, normalized by the intensity of the incoming beam. $E_{\mathrm{Ph}}$ is the photon energy of the incoming beam, and $R\left(E_{P h}\right)$ and $\nu\left(E_{P h}\right)$ are the reflectivity of the sample and the phase of the standing wave field at this energy, respectively. Since $R\left(E_{\mathrm{Ph}}\right)$ and $\nu\left(E_{\mathrm{Ph}}\right)$ follow from the dynamic theory of diffraction, the $E_{\mathrm{ph}}$-dependence of $Y$ is purely determined by the parameters $F^{\mathrm{H}}$ and $P^{\mathrm{H}}$, which thus can be obtained by fitting the above equation to experimentally obtained yield curves. These two parameters can then directly be linked to the geometric structure of the sample, since $P^{\mathrm{H}}$ represents the average position of the studied species relative to the nearest Bragg plane, in units of the Bragg spacing. The second parameter, $F^{\mathrm{H}}$, indicates how well-ordered the species is, with a value of 1 meaning that all atoms of this species lie at precisely the same distance from the nearest Bragg plane and a value of 0 typically indicating complete disorder.

In this work, the amplitude of the X-ray interference field at the atomic species of interest was tracked by recording XPS spectra of the $\mathrm{C} 1 \mathrm{~s}, \mathrm{O} 1 \mathrm{~s}$, and $\mathrm{K} 2 \mathrm{p}$ photoemission signals as the incident beam energy was scanned through the Bragg condition. Subsequently, these XPS spectra were analyzed with core level models to obtain the yield profiles of individual chemical species using CasaXPS 2.3.16 PR 1.6. The fits of the yield curves were performed by means of TORRICELLI. All measurements were performed at beamline I09 of the Diamond Light Source, using a Scienta R4000 electron analyzer mounted at $90^{\circ}$ relative to the incoming X-ray beam. Since in this geometry, nondipolar effects are expected to be minimal, no correction for nondipolar effects has been applied. The films were carefully checked for radiation damage prior to and after each XSW scan. Significant degradation can be excluded at least on the time scale of the experiments. For a more elaborate explanation of the experimental procedure, used core-level models, and the results of this NIXSW analysis refer to the Supporting Information.

Density-Functional Theory Calculations. The optimized adsorption geometries were obtained in our previous work ${ }^{19}$ by means of DFT calculations using the Vienna $a b$ Initio Simulation Package (VASP) ${ }^{66}$ in conjunction with GADGET. ${ }^{67}$ The GGA functional as proposed by Purdew, Burke, and Ernzerhof (PBE) ${ }^{68}$ was employed and the van der Waals interactions were included as described by the vdW ${ }^{\text {surf }}$ method. ${ }^{62}$

A complication arising from the experimentally observed point-online growth is that such a situation is a priori not compatible with the required periodic boundary conditions. This problem can, however, be fixed by choosing suitable supercells in combination with a minute deformation of the substrate (see Figure 1), which for the present system has been tested to have little impact on the obtained results. ${ }^{19}$ Consequently, while constructing the supercells for $\mathrm{K}_{2} \mathrm{PTCDA} /$ $\mathrm{Ag}(111)$ and $\mathrm{K}_{4} \mathrm{PTCDA} / \mathrm{Ag}(111)$ we decided to keep the distances between molecules at the values measured with high-resolution scanning tunneling hydrogen microscopy (STHM) and distortioncorrected LEED and instead slightly changed the Ag lattice constants in order to arrive at unit cells for which the epitaxy matrix contains only integer entries. ${ }^{19}$ For PTCDA $/ \operatorname{Ag}(111)$, since the monolayer was commensurate with the substrate in the experiments, ${ }^{22}$ the PBE lattice constant was used. All slabs consisted of five layers of atoms, where the top two layers were allowed to relax. Please refer to ref 19 for more details of the geometrical optimization.

The structures obtained from VASP were further analyzed using the Quantum ESPRESSO suite, ${ }^{69}$ where ultrasoft pseudopotentials generated with the Rappe, Rabe, Kaxiras, and Joannopoulos scheme ${ }^{70}$ were used with a plane-wave cutoff of $367.35 \mathrm{eV}$ (27 Ry). The density of states was calculated with Gaussian broadenings of 0.11 and $0.02 \mathrm{eV}$ (FWHM) for PTCDA/Ag(111), $\mathrm{K}_{2}$ PTCDA/Ag(111), and $\mathrm{K}_{4} \mathrm{PTCDA} / \mathrm{Ag}(111)$, by using off- $\Gamma$ Monkhorst-Pack $k$-points grids $^{71}$ of $6 \times 6 \times 1,10 \times 6 \times 1$, and $6 \times 6 \times 1$, respectively. While analyzing the band structure (see Figure 5 ), in order to identify the molecular contributions to the fairly large number of bands depicted as thin lines, we superimposed a $k$-resolved density of states (KDOS) projected onto the atomic states belonging to the molecule: $\operatorname{KDOS}(k, E)=\sum_{\phi \in \text { mol }} \sum_{n}\left|\left\langle\phi \mid \psi_{n k}\right\rangle\right|^{2} \delta\left(E-\epsilon_{n k}\right) ; \phi$ are the atomcentered functions used for the Löwdin projections, the sum runs over the atoms of a molecule and $\psi_{n k}$ and $\epsilon_{n k}$ are the Kohn-Sham eigenstates and eigenvalues, respectively. An additional summation over $k$ in the surface Brillouin zone produces the molecular DOS shown in Figure 3. Analyzing the KDOS is conceptually equivalent to plotting the bands with colors depending on the amplitude on a specific portion of the system, ${ }^{56,57}$ but it allows us to analyze cases characterized by dense bands (or even energy continua; see ref 72 for an example). In practice, the $\delta$-function in the above equation was replaced by a Gaussian with the same width as used when plotting the DOS (0.11 eV FWHM); since absolute values of the KDOS depend on the broadening, we normalize it to the maximum value assumed by the broadening function $(8.3 / \mathrm{eV} \times 2$ for spin multiplicity) so that a nondegenerate state $\psi_{n k}$ fully belonging to the molecule has a maximum $\operatorname{KDOS}(k, E)=1$. Figures 1 and 7 have been produced by using the program XCrySDen. ${ }^{7}$

In order to calculate the optical absorption spectra of Figure 4 we adopted the Yambo software ${ }^{74}$ to evaluate the independent particle response from PBE Kohn-Sham orbitals. ${ }^{75}$ Since Yambo can handle neither ultrasoft pseudopotentials nor PAW potentials, additional calculations of the electronic ground state were performed as a preliminary step using the same atomic positions and $k$-point meshes, but norm-conserving pseudopotentials (requiring a higher cutoff of around $680 \mathrm{eV}$ ). We included the empty states in an energy range up to $10 \mathrm{eV}$ above the Fermi energy.

The simulations of individual molecules employing open boundary conditions were conducted using Gaussian09, revision D.01, employing PBE exchange and correlation and a $6-311 \mathrm{G}(\mathrm{d}, \mathrm{p})^{77}$ basis set. Geometries were optimized using standard convergence criteria, and excited-state properties were obtained employing TD-DFT using the same functional and the ground-state geometries. ${ }^{78}$

Finally, we note that in Figure 3 we observe even a quantitative agreement between calculated Kohn-Sham energies and measured ionization energies. While semilocal DFT is typically capable of correctly describing trends, this quantitative agreement between theory and experiment is somewhat surprising considering that when solely considering Kohn-Sham orbital energies one suffers from selfinteraction errors and from the fact that screening effects at the surface are neglected. Still, a quantitative agreement between KohnSham orbital energies and UPS features is not uncommon, ${ }^{79}$ and we attribute that at least in part to a fortuitous cancellation of the abovementioned errors for flat-lying adsorbates. 


\section{ASSOCIATED CONTENT}

\section{S Supporting Information}

The Supporting Information is available free of charge on the ACS Publications website at DOI: 10.1021/acsnano.7b05828.

Determination of the intrinsic width of PDOS peaks; plots showing the integrated local density of states; details of molecule-based simulations and those of adsorbed layer with calculated Kohn-Sham orbital energies; explanation of the LUMO splitting; KDOS plots for adsorbed monolayers without $\mathrm{K}$ atoms; additional charge rearrangement plots; details of experimental methods; extraction of dielectric function from the DRS; details of NIXSW measurements; comparison of experimental and calculated changes in work function (PDF)

\section{AUTHOR INFORMATION}

\section{Corresponding Authors}

*E-mail: guido.fratesi@unimi.it.

*E-mail: torsten.fritz@uni-jena.de.

*E-mail: egbert.zojer@tugraz.at.

\section{ORCID}

Roman Forker: 0000-0003-0969-9180

Guido Fratesi: 0000-0003-1077-7596

Egbert Zojer: 0000-0002-6502-1721

\section{Author Contributions}

$\triangle_{\text {A. Baby and }}$ M. Gruenewald contributed equally.

Notes

The authors declare no competing financial interest.

\section{ACKNOWLEDGMENTS}

A.B. acknowledges the supercomputing time at CINECA, Italy. We thank Serguei Soubatch, Ina Krieger, Norman Haag, TienLin Lee, and Pardeep Kumar Thakur for their support during the NIXSW beam time, as well as David McCue for his excellent technical assistance. The authors are grateful to Oliver T. Hofmann, S. S. Harivyasi, Elsiabeth Wruss, and Elisabeth Verwüster for stimulating discussions and support in performing the simulations. Financial support by the Austrian Science Fund (FWF), P24666-N20, and by the Deutsche Forschungsgemeinschaft via grant FO $770 / 2-1$ is acknowledged. C.Z. acknowledges funding from the Carl-Zeiss-Stiftung. The computational studies presented have in part been achieved using the Vienna Scientific Cluster (VSC).

\section{REFERENCES}

(1) Haddon, R. C. Electronic Structure, Conductivity, and Superconductivity of Alkali Metal Doped $\mathrm{C}_{60}$. Acc. Chem. Res. 1992, 25, 127-133.

(2) Mitsuhashi, R.; Suzuki, Y.; Yamanari, Y.; Mitamura, H.; Kambe, T.; Ikeda, N.; Okamoto, H.; Fujiwara, A.; Yamaji, M.; Kawasaki, N.; Maniwa, Y.; Kubozono, Y. Superconductivity in Alkali-Metal-Doped Picene. Nature 2010, 464, 76-79.

(3) Hebard, A. F.; Rosseinsky, M. J.; Haddon, R. C.; Murphy, D. W.; Glarum, S. H.; Palstra, T. T. M.; Ramirez, A. P.; Kortan, A. R. Superconductivity at $18 \mathrm{~K}$ in Potassium-Doped $\mathrm{C}_{60}$. Nature 1991, 350, $600-601$.

(4) Tanigaki, K.; Ebbesen, T. W.; Saito, S.; Mizuki, J.; Tsai, J. S.; Kubo, Y.; Kuroshima, S. Superconductivity at $33 \mathrm{~K}$ in $\mathrm{Cs}_{\mathrm{x}} \mathrm{Rb}_{\mathrm{y}} \mathrm{C}_{60}$. Nature 1991, 352, 222-223.
(5) Palstra, T. T. M.; Zhou, O.; Iwasa, Y.; Sulewski, P. E.; Fleming, R. M.; Zegarski, B. R. Superconductivity at $40 \mathrm{~K}$ in Cesium Doped $\mathrm{C}_{60}$. Solid State Commun. 1995, 93, 327-330.

(6) Froudakis, G. E. Why Alkali-Metal-Doped Carbon Nanotubes Possess High Hydrogen Uptake. Nano Lett. 2001, 1, 531-533.

(7) Mulfort, K. L.; Hupp, J. T. Alkali Metal Cation Effects on Hydrogen Uptake and Binding in Metal-Organic Frameworks. Inorg. Chem. 2008, 47, 7936-7938.

(8) Keil, M.; Samorí, P.; dos Santos, D. A.; Birgerson, J.; Friedlein, R.; Dkhissi, A.; Watson, M.; Müllen, K.; Brédas, J. L.; Rabe, J. P.; Salaneck, W. R. High Levels of Alkali-Metal Storage in Thin Films of Hexa-PeriHexabenzocoronene. J. Chem. Phys. 2002, 116, 10854-10860.

(9) Cinchetti, M.; Neuschwander, S.; Fischer, A.; Ruffing, A.; Mathias, S.; Wüstenberg, J.-P.; Aeschlimann, M. Tailoring the Spin Functionality of a Hybrid Metal-Organic Interface by Means of AlkaliMetal Doping. Phys. Rev. Lett. 2010, 104, 217602.

(10) Xing, K. Z.; Fahlman, M.; Lögdlund, M.; Berggren, M.; Inganäs, O.; Andersson, M. R.; Boman, M.; Stafström, S.; Iucci, G.; Bröms, P.; Johansson, N.; Salaneck, W. R. The Electronic Structure of Neutral and Alkali Metal-Doped poly[3-(4-Octylphenyl)thiophene] Studied by Photoelectron Spectroscopy. Synth. Met. 1996, 80, 59-66.

(11) Kido, J.; Matsumoto, T. Bright Organic Electroluminescent Devices Having a Metal-Doped Electron-Injecting Layer. Appl. Phys. Lett. 1998, 73, 2866-2868.

(12) Parthasarathy, G.; Shen, C.; Kahn, A.; Forrest, S. R. Lithium Doping of Semiconducting Organic Charge Transport Materials. J. Appl. Phys. 2001, 89, 4986-4992.

(13) Annese, E.; Fujii, J.; Vobornik, I.; Rossi, G. Electronic Band and Orbital Properties of Cs-Doped Pentacene Thin Films. J. Phys. Chem. C 2012, 116, 2382-2389.

(14) El-Sayed, A.; Borghetti, P.; Goiri, E.; Rogero, C.; Floreano, L.; Lovat, G.; Mowbray, D. J.; Cabellos, J. L.; Wakayama, Y.; Rubio, A.; Ortega, J. E.; de Oteyza, D. G. Understanding Energy-Level Alignment in Donor-Acceptor/Metal Interfaces from Core-Level Shifts. ACS Nano 2013, 7, 6914-6920.

(15) Uchida, T.; Kaneta, S.; Ichihara, M.; Ohtsuka, M.; Otomo, T.; Marx, D. R. Flexible Transparent Organic Light Emitting Devices on Plastic Films with Alkali Metal Doping as Electron Injection Layer. Jpn. J. Appl. Phys. 2005, 44, L282-L284.

(16) Zojer, E.; Cornil, J.; Leising, G.; Brédas, J. L. Theoretical Investigation of the Geometric and Optical Properties of Neutral and Charged Oligophenylenes. Phys. Rev. B: Condens. Matter Mater. Phys. 1999, 59, 7957-7968.

(17) Hotta, S.; Waragai, K. Solid-State Absorption Spectroscopy of Alkyl-Substituted Oligothiophenes. J. Phys. Chem. 1993, 97, 74277434.

(18) Hasegawa, Y.; Yamada, Y.; Sasaki, M. Reordering and Disordering of the Copper Hexadecafluorophthalocyanine $\left(\mathrm{F}_{16} \mathrm{CuPc}\right)$ Monolayer by $\mathrm{K}$ Doping. J. Phys. Chem. C 2014, 118, 24490-24496.

(19) Zwick, C.; Baby, A.; Gruenewald, M.; Verwüster, E.; Hofmann, O. T.; Forker, R.; Fratesi, G.; Brivio, G. P.; Zojer, E.; Fritz, T. Complex Stoichiometry-Dependent Reordering of 3,4,9,10-Perylenetetracarboxylic Dianhydride on $\mathrm{Ag}(111)$ upon K Intercalation. ACS Nano 2016 , 10, 2365-2374.

(20) Ules, T.; Lüftner, D.; Reinisch, E. M.; Koller, G.; Puschnig, P.; Ramsey, M. G. Continuous or Discrete: Tuning the Energy Level Alignment of Organic Layers with Alkali Dopants. Phys. Rev. B: Condens. Matter Mater. Phys. 2016, 94, 205405.

(21) Reinisch, E. M.; Ules, T.; Puschnig, P.; Berkebile, S.; Ostler, M.; Seyller, T.; Ramsey, M. G.; Koller, G. Development and Character of Gap States on Alkali Doping of Molecular Films. New J. Phys. 2014, 16, 023011.

(22) Glöckler, K.; Seidel, C.; Soukopp, A.; Sokolowski, M.; Umbach, E.; Böhringer, M.; Berndt, R.; Schneider, W.-D. Highly Ordered Structures and Submolecular Scanning Tunnelling Microscopy Contrast of PTCDA and DM-PBDCI Monolayers on $\operatorname{Ag}(111)$ and $\mathrm{Ag}(110)$. Surf. Sci. 1998, 405, 1-20. 
(23) Wießner, M.; Hauschild, D.; Schöll, A.; Reinert, F.; Feyer, V.; Winkler, K.; Krömker, B. Electronic and Geometric Structure of the PTCDA/Ag(110) Interface Probed by Angle-Resolved Photoemission. Phys. Rev. B: Condens. Matter Mater. Phys. 2012, 86, 045417.

(24) Duhm, S.; Gerlach, A.; Salzmann, I.; Bröker, B.; Johnson, R. L.; Schreiber, F.; Koch, N. PTCDA on $\mathrm{Au}(111), \mathrm{Ag}(111)$ and $\mathrm{Cu}(111)$ : Correlation of Interface Charge Transfer to Bonding Distance. Org. Electron. 2008, 9, 111-118.

(25) Tautz, F. S.; Eremtchenko, M.; Schaefer, J. A.; Sokolowski, M.; Shklover, V.; Glöckler, K.; Umbach, E. A Comparison of the Chemisorption Behaviour of PTCDA on Different Ag Surfaces. Surf. Sci. 2002, 502-503, 176-184.

(26) Romaner, L.; Nabok, D.; Puschnig, P.; Zojer, E.; AmbroschDraxl, C. Theoretical Study of PTCDA Adsorbed on the Coinage Metal Surfaces, $\mathrm{Ag}(111), \mathrm{Au}(111)$ and $\mathrm{Cu}(111)$. New J. Phys. 2009, 11,053010 .

(27) Forker, R.; Meissner, M.; Fritz, T. Classification of Epitaxy in Reciprocal and Real Space: Rigid versus Flexible Lattices. Soft Matter 2017, 13, 1748-1758.

(28) Forker, R; Gruenewald, M.; Fritz, T. Optical Differential Reflectance Spectroscopy on Thin Molecular Films. Annu. Rep. Prog. Chem., Sect. C: Phys. Chem. 2012, 108, 34-68.

(29) Forker, R; Golnik, C.; Pizzi, G.; Dienel, T.; Fritz, T. Optical Absorption Spectra of Ultrathin PTCDA Films on Gold Single Crystals: Charge Transfer beyond the First Monolayer. Org. Electron. 2009, 10, 1448-1453.

(30) Dienel, T.; Krause, A.; Alle, R.; Forker, R.; Meerholz, K.; Fritz, T. Alkali Metal Doped Organic Molecules on Insulators: Charge Impact on the Optical Properties. Adv. Mater. 2010, 22, 4064-4070.

(31) Gómez, U.; Leonhardt, M.; Port, H.; Wolf, H. C. Optical Properties of Amorphous Ultrathin Films of Perylene Derivatives. Chem. Phys. Lett. 1997, 268, 1-6.

(32) Schneider, M. Vibronische und Optische Eigenschaften Ultradünner Organischer Filme am Beispiel PTCDA/Ag(111). Dissertation, Universität Würzburg 2002.

(33) Zou, Y.; Kilian, L.; Schöll, A.; Schmidt, T.; Fink, R.; Umbach, E. Chemical Bonding of PTCDA on Ag Surfaces and the Formation of Interface States. Surf. Sci. 2006, 600, 1240-1251.

(34) Tautz, F. S.; Eremtchenko, M.; Schaefer, J. A.; Sokolowski, M.; Shklover, V.; Umbach, E. Strong Electron-Phonon Coupling at a Metal/organic Interface: PTCDA/Ag(111). Phys. Rev. B: Condens. Matter Mater. Phys. 2002, 65, 125405.

(35) Fratesi, G.; Motta, C.; Trioni, M. I.; Brivio, G. P.; SánchezPortal, D. Resonant Lifetime of Core-Excited Organic Adsorbates from First Principles. J. Phys. Chem. C 2014, 118, 8775-8782.

(36) Löwdin, P. O. On the Nonorthogonality Problem. Adv. Quantum Chem. 1970, 5, 185-199.

(37) Yu, M.; Trinkle, D. R. Accurate and Efficient Algorithm for Bader Charge Integration. J. Chem. Phys. 2011, 134, 064111.

(38) Romaner, L.; Heimel, G.; Brédas, J.-L.; Gerlach, A.; Schreiber, F.; Johnson, R. L.; Zegenhagen, J.; Duhm, S.; Koch, N.; Zojer, E. Impact of Bidirectional Charge Transfer and Molecular Distortions on the Electronic Structure of a Metal-Organic Interface. Phys. Rev. Lett. 2007, 99, 256801.

(39) Stadtmüller, B.; Lüftner, D.; Willenbockel, M.; Reinisch, E. M.; Sueyoshi, T.; Koller, G.; Soubatch, S.; Ramsey, M. G.; Puschnig, P.; Tautz, F. S.; Kumpf, C. Unexpected Interplay of Bonding Height and Energy Level Alignment at Heteromolecular Hybrid Interfaces. Nat. Commun. 2014, 5, 3685.

(40) Brédas, J. L.; Street, G. B. Polarons, Bipolarons, and Solitons in Conducting Polymers. Acc. Chem. Res. 1985, 18, 309-315.

(41) Bussolotti, F.; Kera, S.; Ueno, N. Potassium Doping of Single Crystalline Pentacene Thin Film. Phys. Rev. B: Condens. Matter Mater. Phys. 2012, 86, 155120.

(42) Mercurio, G.; Bauer, O.; Willenbockel, M.; Fiedler, B.; Sueyoshi, T.; Weiss, C.; Temirov, R.; Soubatch, S.; Sokolowski, M.; Tautz, F. S. Tuning and Probing Interfacial Bonding Channels for a Functionalized Organic Molecule by Surface Modification. Phys. Rev. B: Condens. Matter Mater. Phys. 2013, 87, 121409.
(43) Ferretti, A.; Ruini, A.; Molinari, E.; Caldas, M. J. Electronic Properties of Polymer Crystals: The Effect of Interchain Interactions. Phys. Rev. Lett. 2003, 90, 086401.

(44) Hummer, K.; Puschnig, P.; Ambrosch-Draxl, C. Lowest Optical Excitations in Molecular Crystals: Bound Excitons versus Free Electron-Hole Pairs in Anthracene. Phys. Rev. Lett. 2004, 92, 147402.

(45) Rohlfing, M.; Louie, S. G. Electron-Hole Excitations in Semiconductors and Insulators. Phys. Rev. Lett. 1998, 81, 2312-2315.

(46) Rangel, T.; Berland, K.; Sharifzadeh, S.; Brown-Altvater, F.; Lee, K.; Hyldgaard, P.; Kronik, L.; Neaton, J. B. Structural and ExcitedState Properties of Oligoacene Crystals from First Principles. Phys. Rev. B: Condens. Matter Mater. Phys. 2016, 93, 115206.

(47) Mowbray, D. J.; Migani, A. Optical Absorption Spectra and Excitons of Dye-Substrate Interfaces: Catechol on $\mathrm{TiO}_{2}(110)$. J. Chem. Theory Comput. 2016, 12, 2843-2852.

(48) Wüsten, J.; Berger, S.; Salomo, M.; Mönnich, A.; Bauer, M.; Lach, S.; Aeschlimann, M.; Ziegler, C. Hot-Electron Dynamics in Thin Films of Sodium-Doped Perylene-3,4,9,10-Tetracarboxylic Dianhydride. Phys. Rev. B: Condens. Matter Mater. Phys. 2008, 78, 195326.

(49) Egger, D. A.; Ruiz, V. G.; Saidi, W. A.; Bučko, T.; Tkatchenko, A.; Zojer, E. Understanding Structure and Bonding of Multilayered Metal-Organic Nanostructures. J. Phys. Chem. C 2013, 117, 30553061.

(50) Körzdörfer, T.; Kümmel, S.; Marom, N.; Kronik, L. When to Trust Photoelectron Spectra from Kohn-Sham Eigenvalues: The Case of Organic Semiconductors. Phys. Rev. B: Condens. Matter Mater. Phys. 2009, 79, 201205.

(51) Matthes, L.; Pulci, O.; Bechstedt, F. Influence of out-of-Plane Response on Optical Properties of Two-Dimensional Materials: First Principles Approach. Phys. Rev. B: Condens. Matter Mater. Phys. 2016, 94, 205408.

(52) Möbus, M.; Karl, N.; Kobayashi, T. Structure of PeryleneTetracarboxylic-Dianhydride Thin Films on Alkali Halide Crystal Substrates. J. Cryst. Growth 1992, 116, 495-504.

(53) Donati, F.; Fratesi, G.; Passoni, M.; Casari, C. S.; Mairov, A.; Bottani, C. E.; Trioni, M. I.; Li Bassi, A. Strain Effect on Local Electronic Properties of Fe Nanoislands Grown on Au(111). Phys. Rev. B: Condens. Matter Mater. Phys. 2011, 83, 153404.

(54) Sirotti, F.; Beaulieu, N.; Bendounan, A.; Silly, M. G.; Chauvet, C.; Malinowski, G.; Fratesi, G.; Véniard, V.; Onida, G. Multiphoton kResolved Photoemission from Gold Surface States with 800-nm Femtosecond Laser Pulses. Phys. Rev. B: Condens. Matter Mater. Phys. 2014, 90, 035401.

(55) Löwdin, P.-O. On the Non-Orthogonality Problem Connected with the Use of Atomic Wave Functions in the Theory of Molecules and Crystals. J. Chem. Phys. 1950, 18, 365-375.

(56) Kraberger, G. J.; Egger, D. A.; Zojer, E. Tuning the Electronic Structure of Graphene through Collective Electrostatic Effects. Adv. Mater. Interfaces 2015, 2, 1500323.

(57) Giofré, D.; Ceresoli, D.; Fratesi, G.; Trioni, M. I. Electronic Transport in B-N Substituted Bilayer Graphene Nanojunctions. Phys. Rev. B: Condens. Matter Mater. Phys. 2016, 93, 205420.

(58) Zegenhagen, J. Surface Structure Determination with X-Ray Standing Waves. Surf. Sci. Rep. 1993, 18, 199-271.

(59) Woodruff, D. P. Surface Structure Determination Using X-Ray Standing Waves. Rep. Prog. Phys. 2005, 68, 743-798.

(60) Hauschild, A.; Karki, K.; Cowie, B. C. C.; Rohlfing, M.; Tautz, F. S.; Sokolowski, M. Molecular Distortions and Chemical Bonding of a Large $\pi$-Conjugated Molecule on a Metal Surface. Phys. Rev. Lett. 2005, 94, 036106.

(61) Hauschild, A.; Temirov, R.; Soubatch, S.; Bauer, O.; Schöll, A.; Cowie, B. C. C.; Lee, T.-L.; Tautz, F. S.; Sokolowski, M. NormalIncidence X-Ray Standing-Wave Determination of the Adsorption Geometry of PTCDA on $\operatorname{Ag}(111)$ : Comparison of the Ordered Room-Temperature and Disordered Low-Temperature Phases. Phys. Rev. B: Condens. Matter Mater. Phys. 2010, 81, 125432.

(62) Ruiz, V. G.; Liu, W.; Zojer, E.; Scheffler, M.; Tkatchenko, A. Density-Functional Theory with Screened van der Waals Interactions 
for the Modeling of Hybrid Inorganic-Organic Systems. Phys. Rev. Lett.

2012, 108, 146103.

(63) Fratesi, G. Potential Energy Surface of Alkali Atoms Adsorbed on $\mathrm{Cu}(001)$. Phys. Rev. B: Condens. Matter Mater. Phys. 2009, 80, 045422 .

(64) Musket, R. G.; McLean, W.; Colmenares, C. A.; Makowiecki, D. M.; Siekhaus, W. J. Preparation of Atomically Clean Surfaces of Selected Elements: A Review. Appl. Surf. Sci. 1982, 10, 143-207.

(65) Jiang, Y.; Pillai, S.; Green, M. A. Realistic Silver Optical Constants for Plasmonics. Sci. Rep. 2016, 6, 30605.

(66) Kresse, G.; Furthmüller, J. Efficient Iterative Schemes for $A b$ Initio Total-Energy Calculations Using a Plane-Wave Basis Set. Phys. Rev. B: Condens. Matter Mater. Phys. 1996, 54, 11169-11186.

(67) Bučko, T.; Hafner, J.; Ángyán, J. G. Geometry Optimization of Periodic Systems Using Internal Coordinates. J. Chem. Phys. 2005, 122, 124508.

(68) Perdew, J. P.; Burke, K.; Ernzerhof, M. Generalized Gradient Approximation Made Simple. Phys. Rev. Lett. 1996, 77, 3865-3868.

(69) Giannozzi, P.; Baroni, S.; Bonini, N.; Calandra, M.; Car, R.; Cavazzoni, C.; Ceresoli, D.; Chiarotti, G. L.; Cococcioni, M.; Dabo, I.; Dal Corso, A.; de Gironcoli, S.; Fabris, S.; Fratesi, G.; Gebauer, R.; Gerstmann, U.; Gougoussis, C.; Kokalj, A.; Lazzeri, M.; Martin-Samos, L.; et al. QUANTUM ESPRESSO: A Modular and Open-Source Software Project for Quantum Simulations of Materials. J. Phys.: Condens. Matter 2009, 21, 395502.

(70) Rappe, A. M.; Rabe, K. M.; Kaxiras, E.; Joannopoulos, J. D. Optimized Pseudopotentials. Phys. Rev. B: Condens. Matter Mater. Phys. 1990, 41, 1227-1230.

(71) Pack, J. D.; Monkhorst, H. J. “Special Points for Brillouin-Zone Integrations”-a Reply. Phys. Rev. B: Condens. Matter Mater. Phys. 1977, 16, 1748-1749.

(72) Ishida, H.; Hamamoto, Y.; Morikawa, Y.; Minamitani, E.; Arafune, R; Takagi, N. Electronic Structure of the $4 \times 4$ Silicene Monolayer on Semi-Infinite Ag(111). New J. Phys. 2015, 17, 015013.

(73) Kokalj, A. XCrySDen-A New Program for Displaying Crystalline Structures and Electron Densities. J. Mol. Graphics Modell. 1999, 17, 176-179.

(74) Marini, A.; Hogan, C.; Grüning, M.; Varsano, D. Yambo: An Ab Initio Tool for Excited State Calculations. Comput. Phys. Commun. 2009, 180, 1392-1403.

(75) Onida, G.; Reining, L.; Rubio, A. Electronic Excitations: Density-Functional versus Many-Body Green's-Function Approaches. Rev. Mod. Phys. 2002, 74, 601-659.

(76) Frisch, M. J.; Trucks, G. W.; Schlegel, H. B.; Scuseria, G. E.; Robb, M. A.; Cheeseman, J. R.; Scalmani, G.; Barone, V.; Mennucci, B.; Petersson, G. A.; Nakatsuji, H.; Caricato, M.; Li, X.; Hratchian, H. P.; Izmaylov, A. F.; Bloino, J.; Zheng, G.; Sonnenberg, J. L.; Hada, M.; Ehara, M.; et al. Gaussian 09, Revision D.01; Gaussian, Inc.: Wallingford, CT, 2013.

(77) Blaudeau, J.-P.; McGrath, M. P.; Curtiss, L. A.; Radom, L. Extension of Gaussian-2 (G2) Theory to Molecules Containing ThirdRow Atoms K and Ca. J. Chem. Phys. 1997, 107, 5016-5021.

(78) Gross, E. K. U.; Kohn, W. Time-Dependent Density-Functional Theory. Adv. Quantum Chem. 1990, 21, 255-291.

(79) Huang, Y. L.; Wruss, E.; Egger, D. A.; Kera, S.; Ueno, N.; Saidi, W. A.; Bucko, T.; Wee, A. T. S.; Zojer, E. Understanding the Adsorption of $\mathrm{CuPc}$ and $\mathrm{ZnPc}$ on Noble Metal Surfaces by Combining Quantum-Mechanical Modelling and Photoelectron Spectroscopy. Molecules 2014, 19, 2969-2992. 


\section{Supporting Information for:}

\section{Fully Atomistic Understanding of the Electronic and Optical Properties of a Prototypical Doped Charge-Transfer Interface}

Anu Baby ${ }^{\S, 2}$ Marco Gruenewald ${ }^{\S}{ }^{3}$ Christian Zwick, ${ }^{3}$ Felix Otto, ${ }^{3}$ Roman Forker, ${ }^{3}$ Gerben van Straaten, ${ }^{4,5}$ Markus Franke,, ${ }^{4,5}$ Benjamin Stadtmüller, ${ }^{6,7}$ Christian Kumpf, ${ }^{4,5}$ Gian Paolo Brivio, ${ }^{2}$ Guido Fratesi*, ${ }^{8,2}$ Torsten Fritz* $^{3}$ and Egbert Zojer*1

${ }^{1}$ Institute of Solid State Physics, NAWI Graz, Graz University of Technology, Petersgasse 16, 8010 Graz, Austria

${ }^{2}$ Department of Materials Science, University of Milano-Bicocca, Via R. Cozzi 55, 20125 Milano, Italy

${ }^{3}$ Institute of Solid State Physics, Friedrich Schiller University Jena, Helmholtzweg 5, 07743 Jena, Germany

${ }^{4}$ Peter Grünberg Institut (PGI-3), Forschungszentrum Jülich, 52425 Jülich, Germany

${ }^{5}$ Jülich Aachen Research Alliance (JARA) - Fundamentals of Future Information Technology, 52425 Jülich, Germany

${ }^{6}$ Department of Physics and Research Center OPTIMAS, University of Kaiserslautern, 67663 Kaiserslautern, Germany

${ }^{7}$ Graduate School of Excellence Materials Science in Mainz, Erwin-Schrödinger-Straße 46, 67663 Kaiserslautern, Germany

${ }^{8}$ Dipartimento di Fisica, Università degli Studi di Milano, Via Celoria 16, 20133 Milano, Italy 


\section{Simulations}

\subsection{Extracting the intrinsic broadening from the calculated DOS}

For extracting the intrinsic (Lorentzian) width of the LUMO-derived states of the PTCDA adsorbate layer on Ag(111), a semi-infinite model for the silver surface would be desirable. ${ }^{1}$ However, given the large surface unit cells, this would add a significant computational effort, so that a simple estimate is performed here, which is rationalized by the relatively large values obtained for the intrinsic widths.

Assuming an intrinsic Lorentzian profile with a full width at half maximum (FWHM) of $\gamma_{1}$, the shape of a molecule-derived peak in the DOS is represented by a Voigt profile resulting from the convolution of the Lorentzian with the extrinsic Gaussian broadening, $\gamma_{\mathrm{g}}$ introduced in the calculations. The latter was set here to an FWHM $=\gamma_{\mathrm{g}}=0.11 \mathrm{eV}$ while computing the PDOS reported in Figure 3 of the main text. Such an extrinsic width has to be discounted from the FWHM that appears in the plotted PDOS (e.g., in Figure 3 of the main text). A small complication arises here as the Lorentzian and Gaussian widths do not sum up linearly to give the total width of the Voigt profile, $\gamma$, but rather its value is smaller than the sum $\gamma_{1}+\gamma_{\mathrm{g}}$.

This is shown in Figure S1 where we report the FWHM of a Voigt profile obtained by a convolution of the extrinsic Gaussian with a Lorentzian, plotted as a function of the FWHM of the Lorentzian, i.e., $\gamma\left(\gamma_{1}\right)$. To obtain the intrinsic width of the LUMO-derived states for the PTCDA adsorbate layer from the total $\gamma$ accessible by the calculations for a specific value of $\gamma_{\mathrm{g}}$, one can invert this relation, and then $\gamma_{1}^{\text {LUMO }}$ is determined such that $\gamma\left(\gamma_{1}^{\text {LUMO }}\right)$ reproduces the determined value of $\gamma$.

We expect this technique to be less sensitive to the numerical details than directly fitting a Lorentzian function to the non-broadened DOS. We remark, however, that the very same conclusions would be derived also by this approach yielding, in our case, values of $\gamma_{1}=0.26 \mathrm{eV}$, $0.14 \mathrm{eV}$, and $0.06 \mathrm{eV}$ for pristine PTCDA, $\mathrm{K}_{2} \mathrm{PTCDA}$, and $\mathrm{K}_{4} \mathrm{PTCDA}$, respectively. 


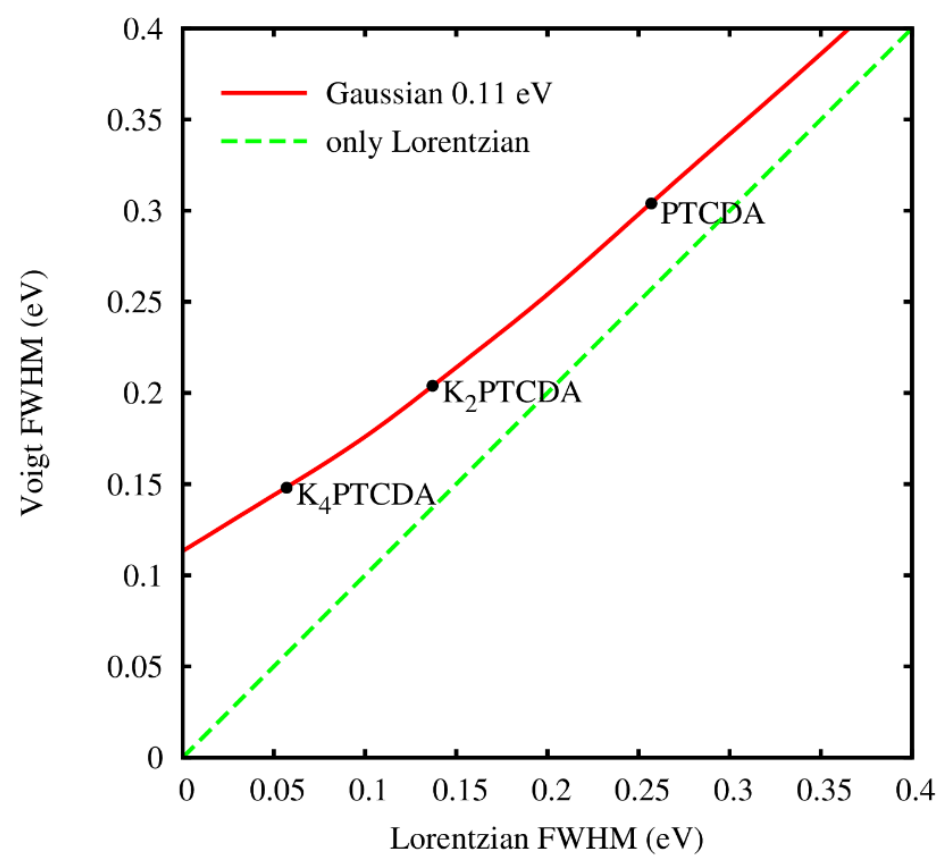

Figure S1: FWHM $\gamma$ of a Voigt profile as a function of the FWHM $\gamma_{l}$ of its Lorentzian contribution (solid red line). The FWHM of the Gaussian is fixed to $\gamma_{\mathrm{g}}=0.11 \mathrm{eV}$. The data points indicate the intrinsic and the total FWHM determined for the LUMO-derived states for different levels of $\mathrm{K}$ doping.

\subsection{Integrated local density of states (ILDOS)}

The densities of states projected onto the molecular region for various levels of $\mathrm{K}$ doping are shown in Figure S2 in analogy to Figure 3b-d in the main manuscript. Here, we additionally plot the local densities of states integrated in a window indicated by shaded gray boxes around the main peaks. A comparison of those LDOSs with the PTCDA gas-phase orbitals (Figure S3) shows that the symmetry and order of the orbitals is neither changed by adsorption on $\mathrm{Ag}(111)$ nor by $\mathrm{K}$ doping. 

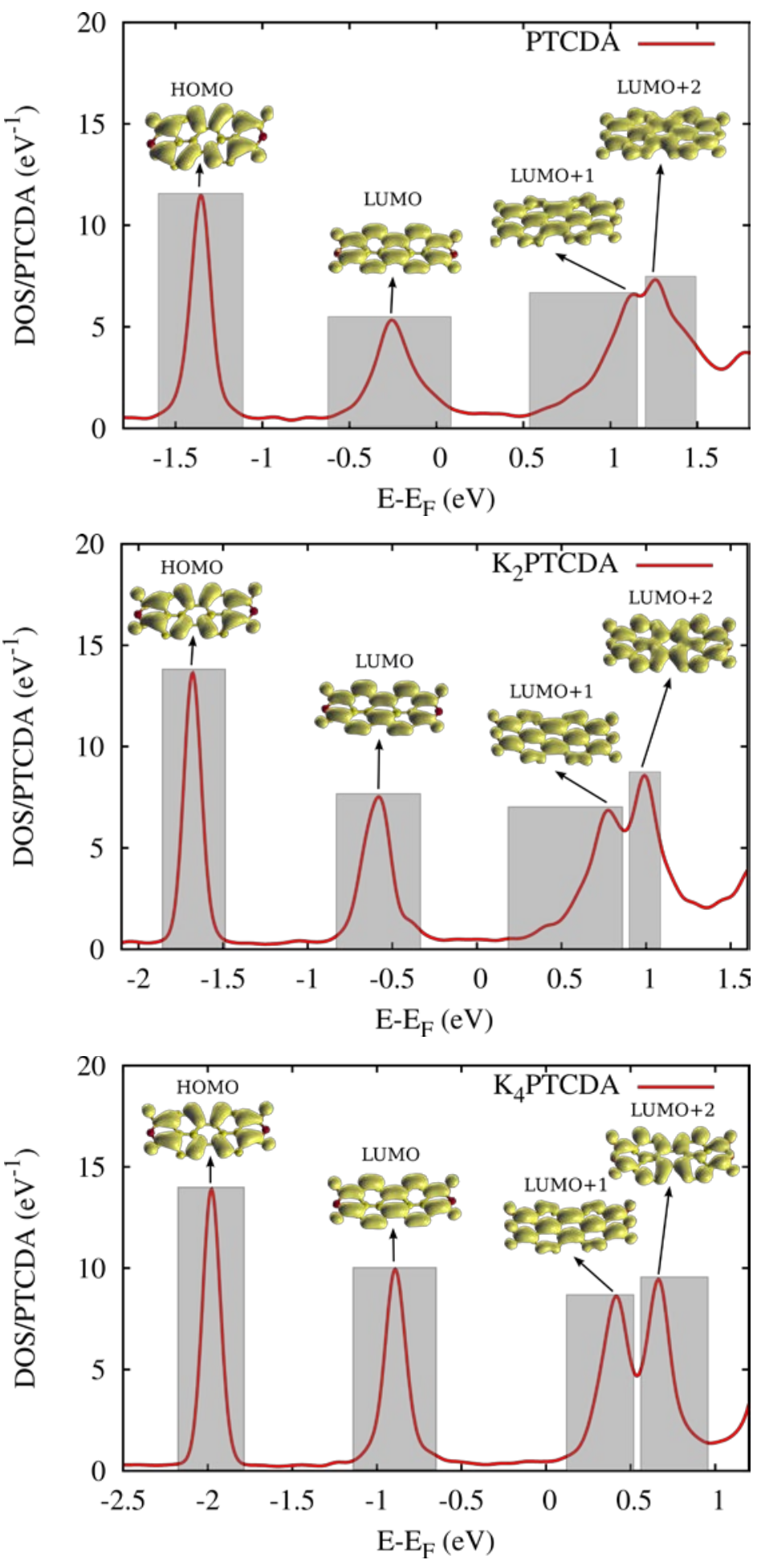

Figure S2: Density of states projected onto the molecular region for various stages of $K$ doping together with local densities of states calculated for energy windows indicated by shaded gray boxes at an isovalue $=0.0008$ e/Bohr ${ }^{3}$ (top: PTCDA/Ag(111); center: $K_{2}$ PTCDA/Ag(111), bottom: $K_{4} P T C D A / A g(111)$. 


\subsection{Further aspects regarding the molecule-based simulations}

Details regarding the molecule-based simulations are provided in the main manuscript. In the following, isodensity plots of the most relevant orbitals (Figure S3), orbital energies and PBEcalculated energy gaps (Table S1, data computed with Gaussian) are provided for the neutral and charged PTCDA. Note that in the following, we will label orbitals and bands according to their occupation in the neutral molecule that they are derived from. This is done to avoid confusions arising from using the same names for symmetry-inequivalent orbitals / bands just as a consequence of different degrees of charging.

Neutral PTCDA

HOMO

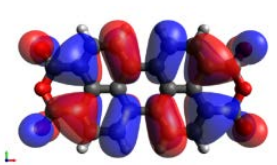

PTCDA Dianion

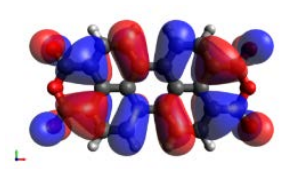

LUMO
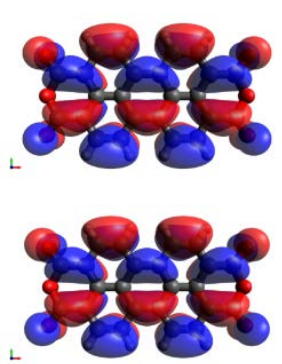

LUMO+1
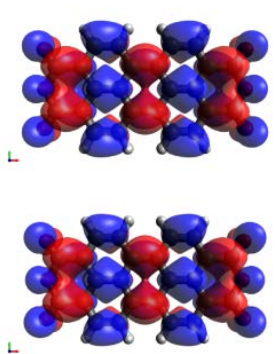

LUMO+2
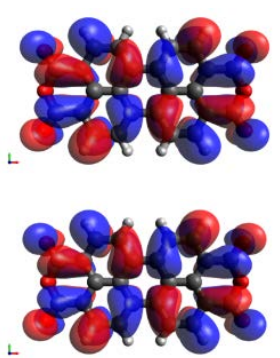

Figure S3: Isodensity plots of molecular orbitals obtained from the Gaussian calculations described in the main text. The plots have been generated using Avogadro 1.1.1. ${ }^{2}$ 
Table S1: DFT-calculated orbital energies of an isolated PTCDA molecule at various charging stages as obtained by using Gaussian. As discussed in the main manuscript $H$ and $L$ refer to the orbitals that have the same symmetry as the HOMO and LUMO of the neutral molecule (consequently, $L$ for the dianion refers to the highest doubly occupied orbital). $\alpha$ and $\beta$ refer to the two spin manifolds.

\begin{tabular}{ccccc}
\hline PTCDA & $\varepsilon_{\mathrm{H}}(\mathrm{eV})$ & $\varepsilon_{\mathrm{L}}(\mathrm{eV})$ & $\varepsilon_{\mathrm{L}+1}(\mathrm{eV})$ & $\varepsilon_{\mathrm{L}+2}(\mathrm{eV})$ \\
\hline neutral & -6.14 & -4.64 & -3.28 & -3.23 \\
anion & $\alpha:-2.71$ & $\alpha:-1.41$ & $\alpha: 0.02$ & $\alpha: 0.12$ \\
& $\beta:-2.54$ & $\beta:-1.22$ & $\beta: 0.13$ & $\beta: 0.24$ \\
\multirow{2}{*}{ dianion } & 0.82 & 1.98 & 3.39 & 3.55 \\
\hline
\end{tabular}

\subsection{DFT-calculated energy gaps for the isolated molecule and the adsorbed monolayer}

Here, we provide a summary of orbital energy gaps for the different PTCDA layers with and without $\mathrm{K}$, both in the adsorbed case and the unsupported free-standing case (Table S2, data computed with Quantum Espresso employing periodic boundary conditions).

Table S2: DFT-calculated energy gaps between the various Kohn-Sham orbitals of PTCDA.

\begin{tabular}{cccccc}
\hline \multicolumn{2}{c}{ Systems } & $\begin{array}{c}\text { HOMO - } \\
\text { LUMO }(\mathrm{eV})\end{array}$ & $\begin{array}{c}\text { LUMO - } \\
\text { LUMO+1 } \\
(\mathrm{eV})\end{array}$ & $\begin{array}{c}\text { LUMO - } \\
\text { LUMO+2 } \\
(\mathrm{eV})\end{array}$ & LUMO+1 - LUMO+2 (eV) \\
\hline \multicolumn{2}{c}{ free molecule } & 1.48 & 1.38 & 1.84 & 0.46 \\
\hline \multirow{2}{*}{$\begin{array}{c}\text { adsorbed } \\
\text { monolayer }\end{array}$} & $\mathrm{K}_{2}$ PTCDA & 1.09 & 1.39 & 1.54 & 0.15 \\
& $\mathrm{~K}_{4}$ PTCDA & 1.09 & 1.35 & 1.56 & 0.21 \\
\hline $\begin{array}{c}\text { free- } \\
\text { standing } \\
\text { monolayer }\end{array}$ & KTCDA & 1.20 & 1.33 & 1.56 & 0.25 \\
& $\mathrm{~K}_{2}$ PTCDA & 1.12 & 1.32 & 1.55 & 0.22 \\
\hline
\end{tabular}




\subsection{Explanation for the shape of the LUMO-derived DOS}

We present an analysis of the shape of the LUMO-derived DOS peak shown in Figure 3b-d for the three phases, by considering their dispersion shown in Figure 5a-c. Focusing on the herringbone PTCDA states in Figure 5a, a green-shaded band between $-0.5 \mathrm{eV}$ and $0.0 \mathrm{eV}$ is found, giving rise to the moderately broad DOS peak in Figure 3b, and arising from a strong hybridization between adsorbate and substrate states at all $k$-points of the surface Brillouin zone. Conversely, the corresponding features in the band structure of $\mathrm{K}_{2}$ PTCDA and $\mathrm{K}_{4} \mathrm{PTCDA}$ (Figure $5 \mathrm{~b}$ and c) are significantly more resolved in energy, and their dispersion can be easily appreciated. In particular, the LUMO-derived features of $\mathrm{K}_{2} \mathrm{PTCDA}$ around $-0.5 \mathrm{eV}$ shows a lower energy at the $\mathrm{K}$ point than at the $\Gamma$ point, which is due to the opposite sign of the LUMO state at the $O$ terminations of facing molecules in the $\mathrm{K}_{2}$ PTCDA geometry (see arrow in Figure S4). Correspondingly, a double-peak structure can be seen in the DOS in Figure 3c. For $\mathrm{K}_{4}$ PTCDA, this behavior is not observed because of the different molecular arrangement.

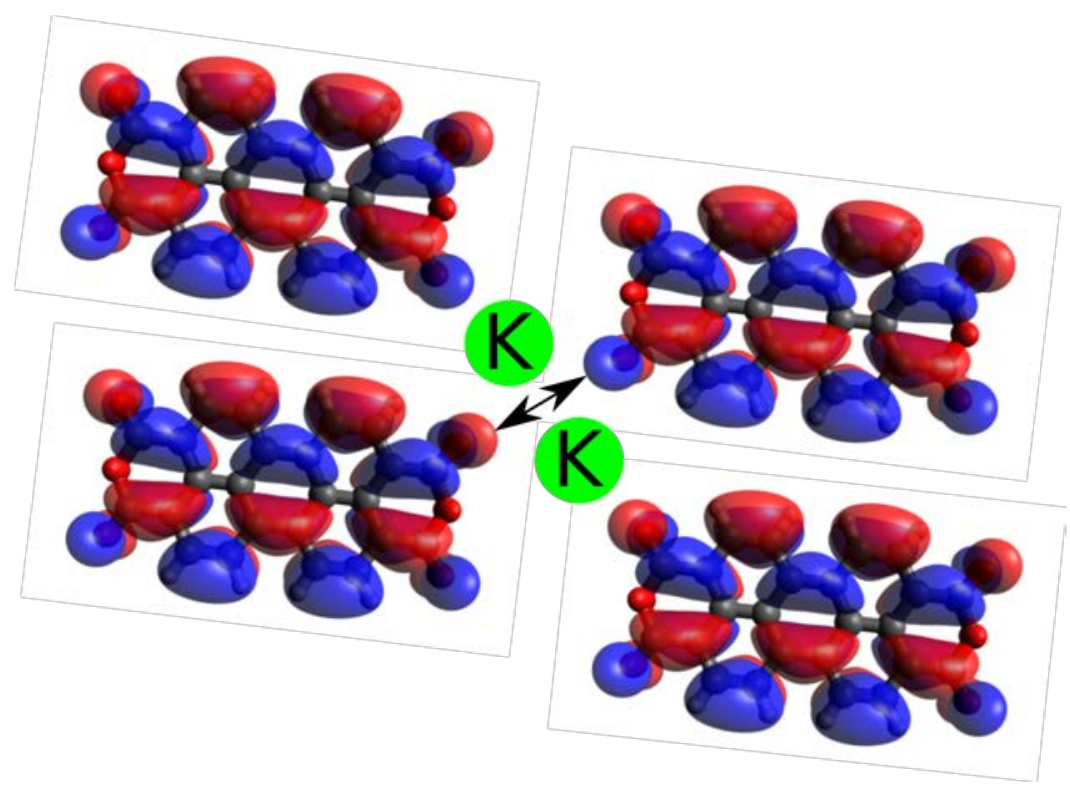

Figure S4: LUMO orbitals of PTCDA with molecules arranged in the structure they adopt in the $K_{2} P T C D A / A g(111)$ system.

\section{6 $k$-projected band structure for $K_{x}$ PTCDA/Ag(111) in the absence of $\mathrm{K}$ atoms}

To determine to what extent the reduced widths of the DOS features in $\mathrm{K}_{2} \mathrm{PTCDA} / \mathrm{Ag}(111)$ compared to PTCDA/Ag(111) are a consequence of geometric deformations and to what extent they 
are caused by the electronic interactions with the $\mathrm{K}$ atoms, we studied the transition from PTCDA/Ag(111) to $\mathrm{K}_{2}$ PTCDA/Ag(111) with two intermediate steps: As a first step we moved the PTCDA molecules from their original herringbone arrangement to the positions they would assume in the $\mathrm{K}_{2} \mathrm{PTCDA} / \mathrm{Ag}(111)$ system, but removed the $\mathrm{K}$ atoms and fully optimized the geometries. This yielded the band-structure and DOS shown in Figures S5a. One sees that this already "sharpens" the KDOS especially for the LUMO-derived peak. The resulting dispersion in $k$, however, increases with the consequence that the overall width of the LUMO-derived feature does not decrease. In fact, it rather increases to an intrinsic width of $0.34 \mathrm{eV}$ compared to the $0.26 \mathrm{eV}$ for herringbone PTCDA/Ag(111).

As a second step, as shown in Figure S5b, the geometry of the PTCDA molecules is set to that calculated for $\mathrm{K}_{2}$ PTCDA/Ag(111) with no $\mathrm{K}$ atoms actually present on the surface. Given the large number of features, the focus is on the LUMO-derived state whose dispersion results in a different energy at $\Gamma$ and $\mathrm{K}$ points of the surface Brillouin zone (also producing the double-peak structure of the LUMO feature in the DOS discussed in the previous Section). Interestingly, this energy splitting remains large and comparable to the one of relaxed molecules of Figure S5a, in spite of the detachment of the carboxylic oxygens from the surface, the lifting of the perylene core, and an apparently missing "link” provided by K atoms between molecules. Only when also adding the K atoms (cf., Figure 5b and Figure 3c in the main text), the dispersion of the LUMO-derived states is significantly reduced. This can be attributed to a change of the inter-molecular coupling within the PTCDA layer caused by the presence of the $\mathrm{K}$ atoms highlighting that it is a combination of geometry-related and electronic effects reducing both the coupling to the metal substrate and the coupling between the PTCDA molecules that gives rise to the sharper spectral features in the $\varepsilon^{\prime \prime}$ spectra of $\mathrm{K}_{2} \mathrm{PTCDA} / \operatorname{Ag}(111)$.

Careful observation identifies the same trend when the $k$-projected bands are calculated for an unrelaxed geometry of $\mathrm{K}_{4} \mathrm{PTCDA}$ in the absence of $\mathrm{K}$ atoms (compare Figure S5c with $\mathrm{K}_{4} \mathrm{PTCDA}$ in Figure $5 \mathrm{c}$ of the main text). Here one should keep in mind that the unit cell contains two molecules, so that the surface Brillouin zone is halved and two features derived from the LUMOs appear in the band dispersion. This again results in a double-peak structure of the DOS in the absence of $\mathrm{K}$ atoms (see Figure $3 \mathrm{~d}$ of the main text), corresponding to the almost flat bands as evinced in Figure $5 c$ of the main text. This result further confirms the interpretations made above. 

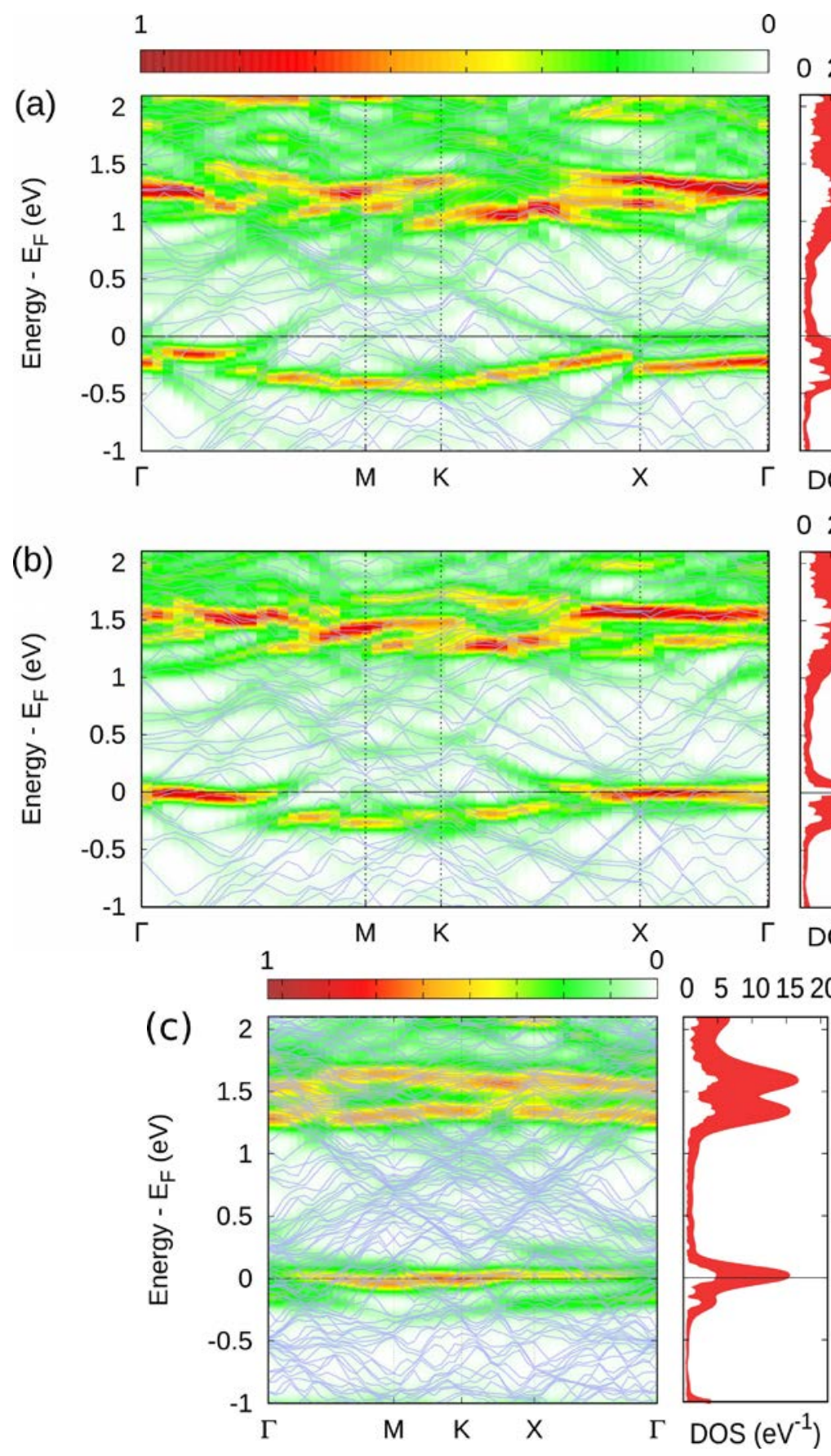

Figure S5. k-projected band structure (left panel) and DOS per PTCDA (right panel) for (a) PTCDA optimized in the $K_{2} P T C D A / A g(111)$ unit cell in the absence of K atoms, (b) PTCDA in the $K_{2} P T C D A / A g(111)$ structure in the absence of $K$ atoms, but in the geometry adopted when K atoms are present, and (c) an analogous plot to (b) but for the $K_{4}$ PTCDA unit cell. 


\subsection{Alternative view on charge rearrangements}

As mentioned in the main manuscript, in an alternative scenario for analyzing the charge rearrangements upon $\mathrm{K}_{\mathrm{x}} \mathrm{PTCDA} / \mathrm{Ag}(111)$ interface formation, one first considers the formation of a hypothetical free standing $\mathrm{K}_{\mathrm{x}} \mathrm{PTCDA}$ layer, which, in a second step, adsorbs onto the $\operatorname{Ag}(111)$ substrate. For the interaction between the K atoms and the PTCDA molecules in a hypothetical freestanding monolayer (first step in the second scenario, see Figure S6a) the charge rearrangement around the oxygen atoms are similar to the ones shown in Figure S7a for the PTCDA layer already bonded to the Ag substrate, i.e., the presence of the Ag substrate does not change the situation qualitatively. The main difference is that in the absence of the Ag substrate the electron transfer into the PTCDA LUMO is more massive, which is not surprising because in that case this orbital is completely empty, while for the molecules adsorbed on the Ag(111) substrate it is already largely filled. When adsorbing the $\mathrm{K}_{2}$ PTCDA monolayer onto the $\mathrm{Ag}(111)$ substrate (step 2 in the present scenario; Figure S6b), one primarily observes a charge transfer towards the $\mathrm{Ag}(111)$ surface which is accompanied by a minor depletion of electron density in the $\pi$-system of the PTCDA molecules.

(a)

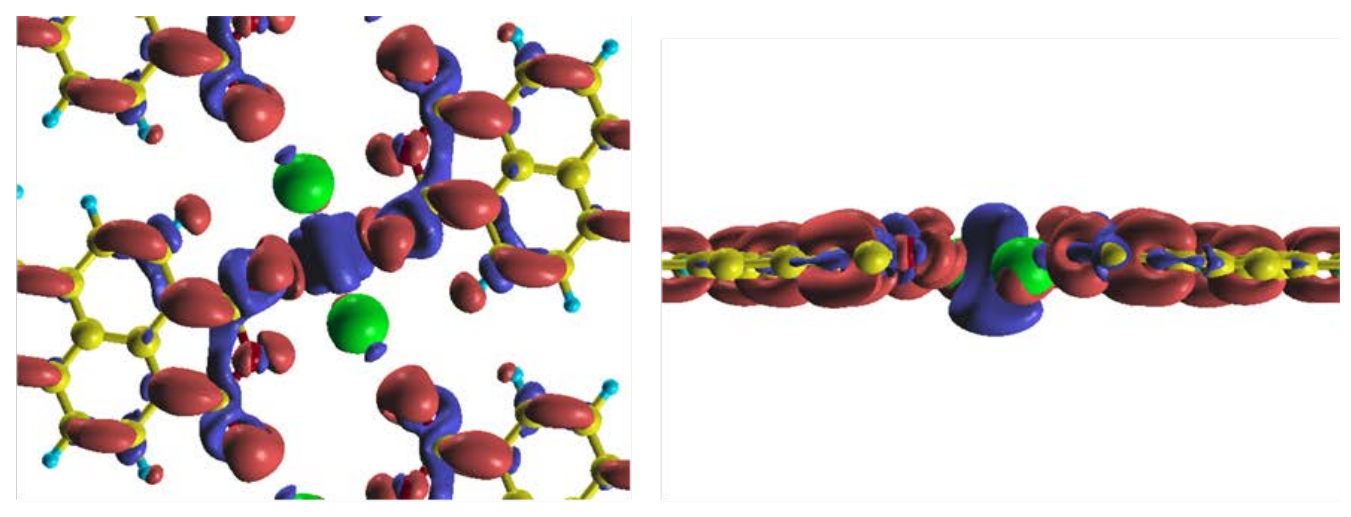

(b)

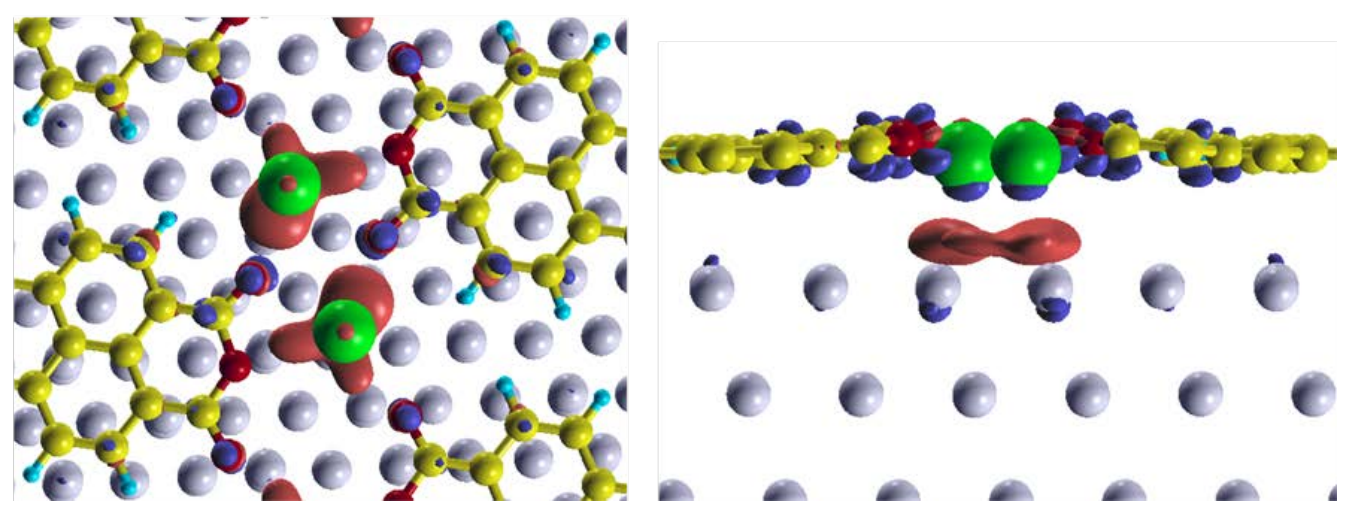

Figure S6: Top and side views for the charge rearrangements occurring upon $K$ doping in the $K_{x} P T C D A / A g(111)$ systems. (a) shows $\rho\left(K_{2} P T C D A\right)-\rho(P T C D A)-\rho\left(K_{2}\right)$, and (b) $\rho\left(K_{2} P T C D A / A g(111)\right)-\rho\left(K_{2} P T C D A\right)-\rho(A g(111))$. Red regions show electron accumulation and 
blue show electron depletion (isovalue $=0.002 \mathrm{e} / \mathrm{Bohr}^{3}$ ). The color code of $\mathrm{C}, \mathrm{O}$, and $\mathrm{H}$ atoms corresponds to that in Fig. 1, while Ag atoms are depicted in gray here.

Finally, for the sake of comparison Figure S7 shows the charge rearrangements for K adsorption onto a pristine $\mathrm{Ag}(111)$ surface.
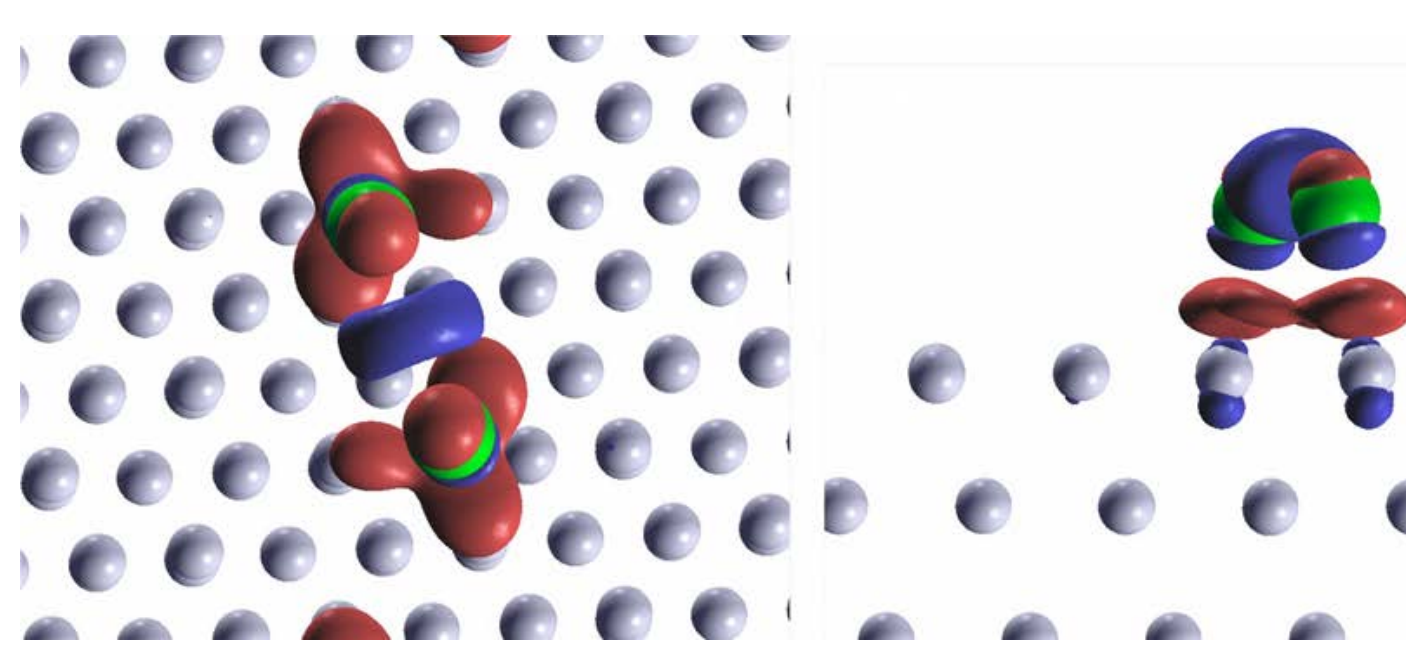

Figure S7: Top and side views for the charge rearrangements occurring upon K-adsorption on $A g(111)\left[\rho\left(K_{2} / A g(111)\right)-\rho\left(K_{2}\right)-\rho(A g(111))\right]$ in the $K_{2} P T C D A / A g(111)$ configuration without PTCDA. Red regions show electron accumulation and blue show electron depletion (isovalue = 0.002 e/Bohr ${ }^{3}$ ). The color code of $C, O$, and $\mathrm{H}$ atoms corresponds to that in Fig. 1, while Ag atoms are depicted in gray here.

\section{Experiments}

\subsection{Optical differential reflectance spectroscopy (DRS) and extraction of}

\section{dielectric function}

We define the measured signal of optical differential reflectance spectroscopy (DRS) as follows:

$$
\operatorname{DRS}(d):=\frac{R(d)-R(0)}{R(0)}
$$


Here $R(d)$ denotes the reflected intensity of the substrate covered with an adsorbate film of thickness $d$, meaning that $R(0)$ stands for the pristine substrate. By performing DRS in situ during the growth of the film one obtains a thickness-dependent spectral quantity $\operatorname{DRS}(d)$. Alternatively, instead of $d$ one can also vary any other quantity $q$ (time, temperature, amount of dopant atoms added to a molecular film, etc.) and will then obtain a signal varying as a function of that quantity $\operatorname{DRS}(q)$. The technique and the setup used by us is explained in detail elsewhere. ${ }^{3}$ Figure S8 shows the DRS data acquired during the deposition of PTCDA on Ag(111) and during the subsequent deposition of potassium.

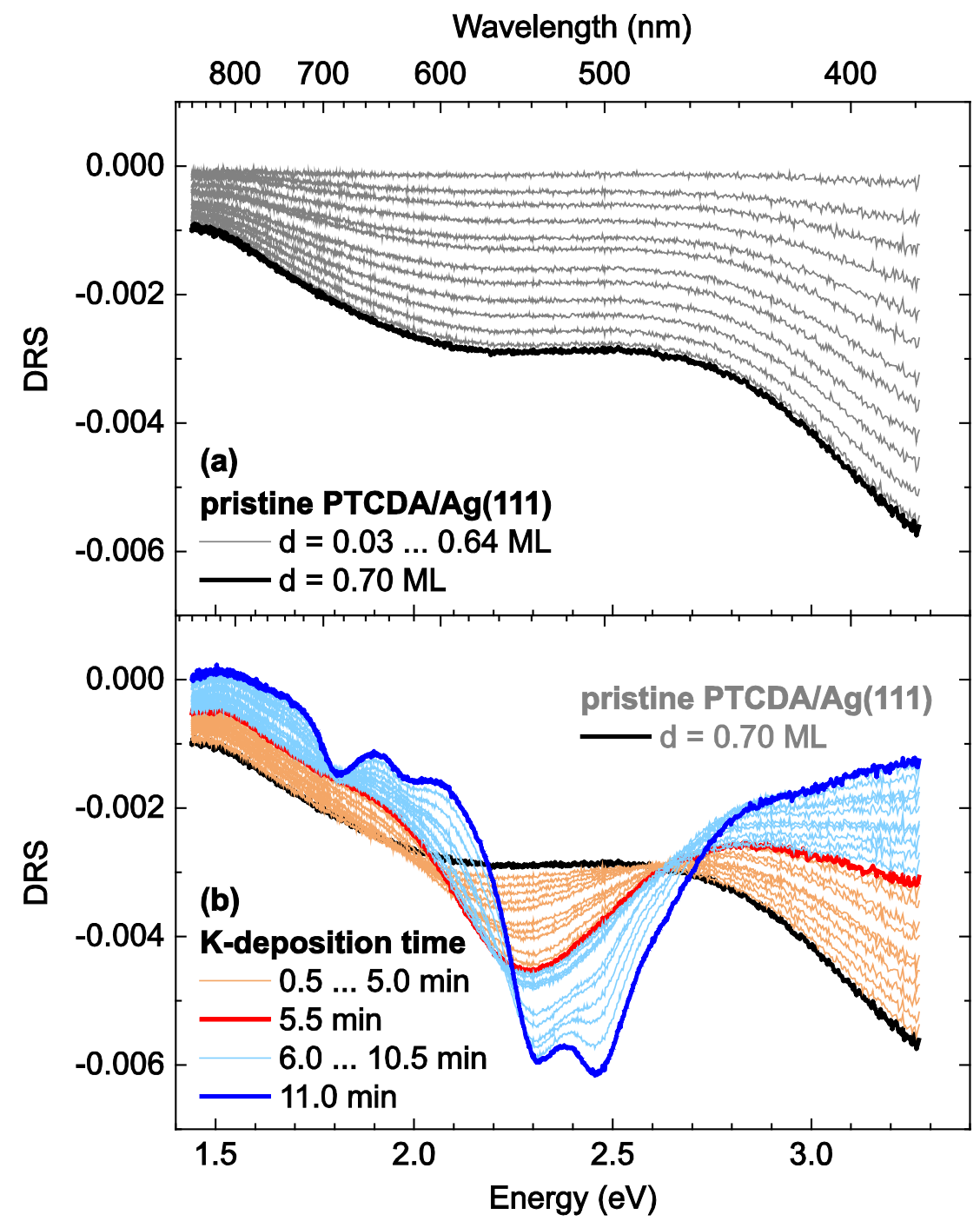

Figure S8: (a) DRS raw data for the deposition of PTCDA onto Ag(111). Note that the DRS signal is essentially proportional to the effective film thickness $d$. (b) DRS raw data of the subsequent $K$ deposition yielding strong spectral changes. The K-doping stages shown in Figure 2 in the main text are highlighted in the same color code for PTCDA/Ag(111) [d =0.70 ML], $K_{2}$ PTCDA/Ag(111)

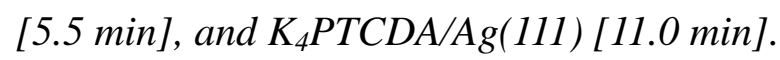


The DRS signal generally contains contributions from both substrate and adsorbate ${ }^{3}$. Therefore, it is necessary to extract the complex dielectric function of the adsorbate film $\tilde{\varepsilon}_{\text {ads }}=\varepsilon_{\text {ads }}^{\prime}-i \cdot \varepsilon_{\text {ads }}^{\prime \prime}$ which can in principle be accomplished by employing a Kramers-Kronig-consistent numerical algorithm published previously. ${ }^{3}$ In this particular case, however, the very broad DR spectra of hybridized PTCDA films do not fully cover an optical transition (i.e., the DRS signal does not reach zero at both ends of the measured spectral range), and thus the prerequisites for the applicability of said algorithm are not met. Nevertheless, it is possible to analytically derive just the imaginary part $\varepsilon_{\text {ads }}^{\prime \prime}$ of the adsorbate dielectric function from the DRS measurements in the present case, as will be described in the following. For this purpose, one needs to make a few reasonable assumptions in order to keep the resulting formulas simple:

1. We consider planar and parallel surfaces and interfaces in the physical layer model.

2. We consider a semi-infinite substrate, i.e., the reflectance of its backside does not contribute to the measured signal. This is clearly fulfilled for opaque substrates such as the massive Ag(111) single crystal used in this study.

3. The propagation direction of the incident light is considered to be perpendicular to the substrate surface. In our experimental setup this is not fully realized - the incident angle is $20^{\circ}$ off the surface normal. However, this does not yield a large error in the data evaluation, bearing in mind that additional factors in the order of $\cos \left(20^{\circ}\right)=0.94 \approx 1$ would then appear in the equations. Neglecting these additional factors results in a quantitative error on the order of a few percent, which we deem acceptable in exchange for the much simpler equations for perpendicular incidence.

4. We consider film thicknesses much smaller than the employed photon wavelengths between roughly 400 and $800 \mathrm{~nm}$. Consequently, only first orders in $d / \lambda$ are taken into account for the analytical linearization of the DRS formula. This is a very reasonable approximation given that $d$ is in the order of $0.3 \mathrm{~nm}$ for monolayers of planar aromatic molecules adsorbed flat-lying on the substrate.

5. Each layer is treated as homogeneous. Of course, a sub-monolayer film cannot be homogenous on a substrate. However, bearing in mind that we have a macroscopic spot diameter $(\approx 1 \mathrm{~mm})$ of the incident light we simultaneously probe covered and uncovered substrate areas. Evidently, for a 0.7 ML adsorbate film the sample area probed by the incident light contains $70 \%$ of single-layer adsorbate domains and 30\% of pristine substrate 
surface, which yields an effective adsorbate film thickness $d$ of 0.7 times the physical film thickness of a full monolayer.

Under these assumptions a linearization of the DRS signal is analytically feasible ${ }^{4}$ and yields:

$$
\mathrm{DRS}=-\frac{8 \pi d}{\lambda} \cdot \frac{\left(1-\varepsilon_{\text {sub }}^{\prime}\right) \cdot \Delta \varepsilon^{\prime \prime}+\varepsilon_{\text {sub }}^{\prime \prime} \cdot \Delta \varepsilon^{\prime}}{\left(1-\varepsilon_{\text {sub }}^{\prime}\right)^{2}+\left(\varepsilon_{\text {sub }}^{\prime \prime}\right)^{2}}
$$

In our DRS setup we use unpolarized light, hence the difference in reflectance in DRS merely stems from the fact that in the initial system "vacuum/substrate" a layer is inserted upon film growth: “vacuum/film/substrate”. Therefore, $\Delta \varepsilon^{\prime \prime}$ here corresponds to $\left[\varepsilon_{\text {ads }}^{\prime \prime}-0\right]$, and $\Delta \varepsilon^{\prime}$ corresponds to $\left[\varepsilon_{\text {ads }}^{\prime}-1\right]$, i.e., just the difference between the dielectric constant of the adsorbate film and that of vacuum (separated in real and imaginary parts). In other words, we can rewrite the equation as:

$$
\mathrm{DRS}=-\frac{8 \pi d}{\lambda} \cdot\left(A_{\mathrm{sub}} \cdot \varepsilon_{\mathrm{ads}}^{\prime \prime}+B_{\mathrm{sub}} \cdot\left[\varepsilon_{\mathrm{ads}}^{\prime}-1\right]\right)
$$

where the optical properties of the bulk substrate are characterized by the spectral functions:

$$
\begin{aligned}
& A_{\text {sub }}=\frac{1-\varepsilon_{\text {sub }}^{\prime}}{\left(1-\varepsilon_{\text {sub }}^{\prime}\right)^{2}+\left(\varepsilon_{\text {sub }}^{\prime \prime}\right)^{2}} \\
& B_{\text {sub }}=\frac{\varepsilon_{\text {sub }}^{\prime \prime}}{\left(1-\varepsilon_{\text {sub }}^{\prime}\right)^{2}+\left(\varepsilon_{\text {sub }}^{\prime \prime}\right)^{2}}
\end{aligned}
$$

We use a recent literature dataset for the complex refractive index $\tilde{n}=n-i \cdot k$ of bulk silver, from which its complex dielectric function $\tilde{\varepsilon}_{\text {sub }}=\varepsilon_{\text {sub }}^{\prime}-i \cdot \varepsilon_{\text {sub }}^{\prime \prime}=\tilde{n}^{2}$ can be obtained. ${ }^{5}$ This dataset is displayed in Figure S9, along with the derived functions $A_{\text {sub }}$ and $B_{\text {sub }}$ according to the above formula. 


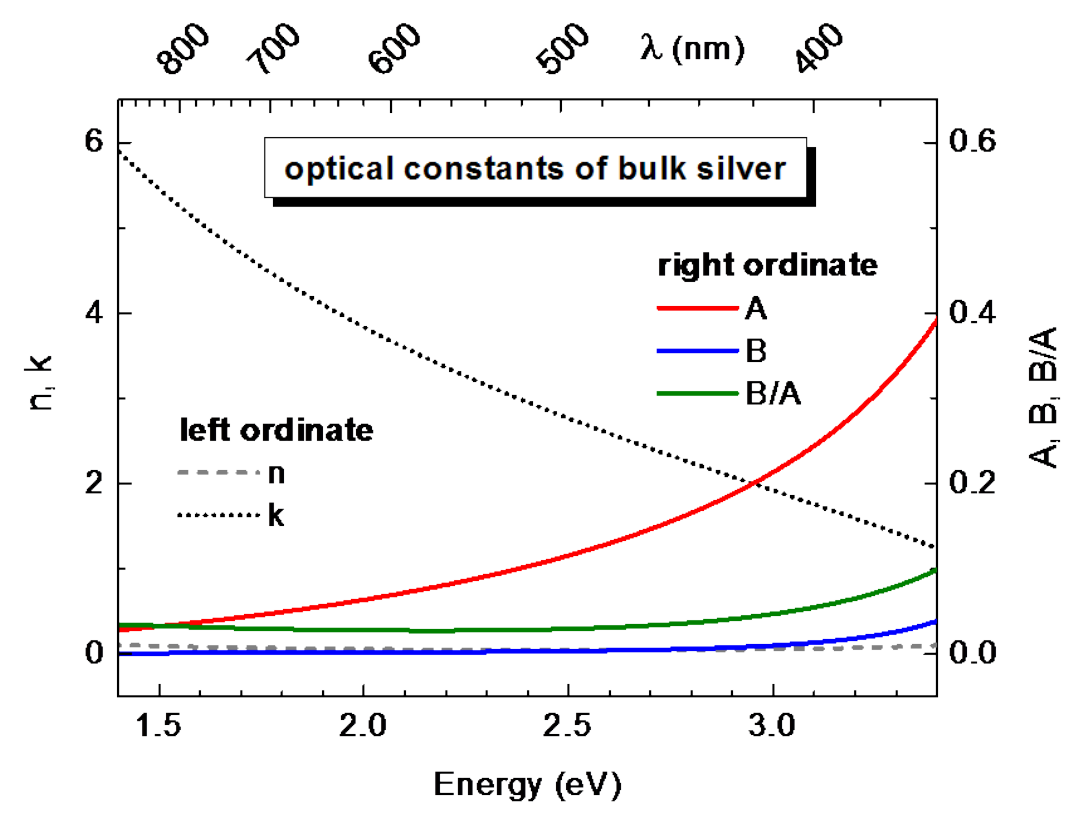

Figure S9: Complex refractive index $\tilde{n}=n-i \cdot k$ (left ordinate) of bulk silver ${ }^{5}$ and the optical functions $A_{\text {sub }}$ and $B_{\text {sub }}$ calculated thereof (right ordinate). The ratio $B_{\text {sub }} / A_{\text {sub }}$ is also plotted.

In the spectral region of interest here, the ratio $B_{\text {sub }} / A_{\text {sub }}$ is on the order of $\approx 0.05$, approaching a value of only 0.10 at $3.4 \mathrm{eV}$. Therefore, $B_{\text {sub }}$ can be neglected in the DRS equation (again causing a quantitative error of just a few percent). The DRS equation then simplifies to:

$$
\mathrm{DRS} \approx-\frac{8 \pi d}{\lambda} \cdot A_{\mathrm{sub}} \cdot \varepsilon_{\mathrm{ads}}^{\prime \prime} \quad \Rightarrow \quad \varepsilon_{\mathrm{ads}}^{\prime \prime} \approx-\mathrm{DRS} \cdot \frac{\lambda}{8 \pi d \cdot A_{\mathrm{sub}}}
$$

Then the measured DRS signal can be directly converted into $\varepsilon_{\text {ads }}^{\prime \prime}$, which is the most significant quantity to characterize the absorption behavior of the adsorbate film. This is shown exemplarily in Fig. S10a, while Fig. S10b provides the full dataset of $\varepsilon$ " spectra corresponding to the DRS data depicted in Fig. S8b.

In contrast to the imaginary part of the dielectric function, the real part $\varepsilon_{\text {ads }}^{\prime}$ is not accessible through this method. It is also not possible to derive $\varepsilon_{\text {ads }}^{\prime}$ from $\varepsilon_{\text {ads }}^{\prime \prime}$ via an a posteriori KramersKronig transformation, owing to the incomplete coverage of the rather broad optical transitions of hybridized molecular films with the limited spectral range available. Due to the fact that the experimentally determined $\varepsilon_{\text {ads }}^{\prime \prime}$ cannot be integrated as a consequence of the missing slopes outside the measurement region, the experimentally determined $\varepsilon_{\text {ads }}^{\prime \prime}$ and the simulated oscillator strengths are not quantitatively comparable. 


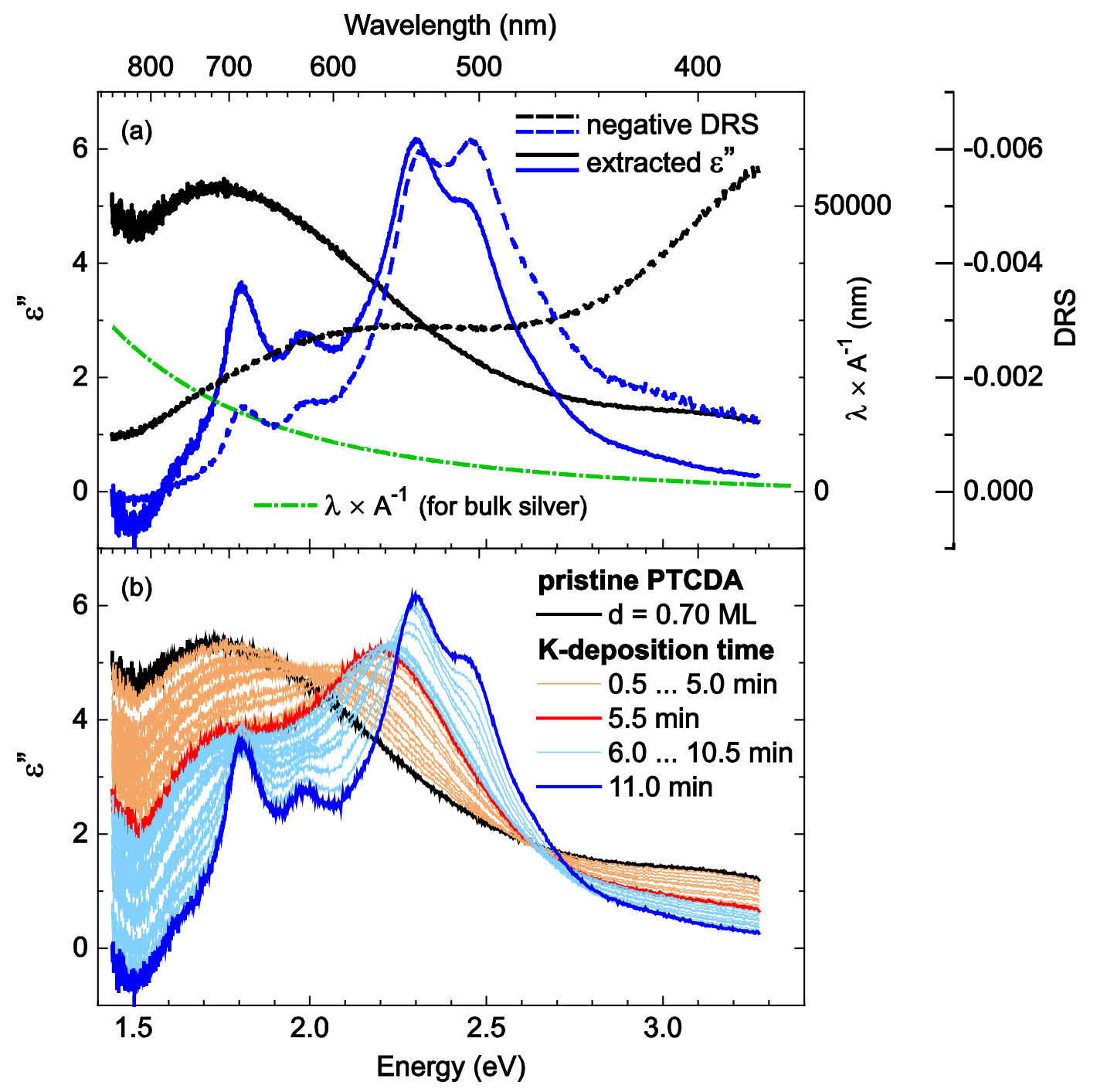

Figure S10: (a) Extraction of $\varepsilon^{\prime \prime}$ spectra (solid lines) from the DRS raw data (dashed lines, separate scale) utilizing the above approximation $\varepsilon_{a d s}^{\prime \prime} \approx-D R S \cdot(8 \pi d)^{-1} \cdot \lambda / A_{\text {sub }}$ for two selected examples: PTCDA/Ag(111) [d=0.70 ML, black curves] and $K_{4} P T C D A / A g(111)$ [11.0 min, blue curves]. The fraction $\lambda / A_{\text {sub }}$ (green dash-dotted line) is derived from the optical constants of bulk silver and has a pronounced wavelength dependence, which results in a different spectral appearance of $\varepsilon_{a d s}^{\prime \prime}$ as compared to the (negative) DRS. (b) Full dataset of $\varepsilon^{\prime \prime}$ spectra corresponding to the DRS data depicted in Fig. S8b. 


\subsection{XPS Measurements}

In order to develop a peak fitting model for the analysis of NIXSW data, we initially recorded reference core-level spectra from all atomic species that are present in the pristine PTCDA/Ag(111) film (0.7 ML) as well as in the doped PTCDA films comprising $\mathrm{K}_{2} \mathrm{PTCDA}$ and $\mathrm{K}_{4} \mathrm{PTCDA}$ complexes (cf. Fig. S11). Our fitting models for the oxygen species are based on Ref. 6; for the carbon species we follow Ref. 7. The peak assignments are shown in the upper insets of Fig. S11.

The peak fitting model well reproduces the C1s and O1s PE spectra of pristine PTCDA/Ag(111) shown in Fig. S11a. We then transferred the peak fitting model to the PE spectra of the K-doped films. For $\mathrm{K}_{2} \mathrm{PTCDA} / \mathrm{Ag}(111)$ shown in Fig. S11b, a doublet of K2p spin-orbit-split peaks ( $\mathrm{K} 1_{1 / 2}^{\mathrm{m}}$ and $\mathrm{K}_{3 / 2}^{\mathrm{m}}$ ), which are energetically separated by $2.74 \mathrm{eV}$, is additionally visible in the high binding energy region. For the $\mathrm{K}_{4} \mathrm{PTCDA} / \mathrm{Ag}(111)$ film shown in Fig. S11c, the K2p emission lines even indicate two inequivalent potassium species, namely potassium incorporated into the PTCDA film $\left(\mathrm{K}_{1 / 2}^{\mathrm{m}}\right.$ and $\left.\mathrm{K} 1_{3 / 2}^{\mathrm{m}}\right)$ and excess potassium $\left(\mathrm{K}_{1 / 2}^{\mathrm{m}}\right.$ and $\left.\mathrm{K} 2_{3 / 2}^{\mathrm{m}}\right)$. The higher binding energy of the excess potassium means stronger oxidization, which suggests that this species directly adsorbs on the $\mathrm{Ag}(111)$ surface. This assignment was further checked by a reference experiment for potassium deposited onto a clean $\mathrm{Ag}(111)$ substrate. We estimate the stoichiometries as 2.5 and 4.6 potassium atoms bound to PTCDA for the reference samples of $\mathrm{K}_{2} \mathrm{PTCDA} / \mathrm{Ag}(111)$ and $\mathrm{K}_{4} \mathrm{PTCDA} / \mathrm{Ag}(111)$, respectively. There is little excess potassium in the first case, i.e., $\mathrm{K} 2 \mathrm{~m} / 2$ and $\mathrm{K} 2 \mathrm{~m} / 2$ peaks cannot be fitted meaningfully to the data in Figure S11b. However, in the second case excess potassium occurs significantly with 3.1 potassium atoms per PTCDA molecule, i.e., in total 7.7 potassium atoms per PTCDA molecule are concluded from the analysis of Figure S11c.

The C1s, K2p, and O1s PE signatures of the potassium-doped PTCDA films were measured for grazing (cf. Fig. S11) and normal emission (not shown). Since no significant differences are observed, it is rather likely that potassium $\left(\mathrm{K} 1_{1 / 2}^{\mathrm{m}}\right.$ and $\left.\mathrm{K} 1_{3 / 2}^{\mathrm{m}}\right)$ is situated in between rather than underneath the molecules. 

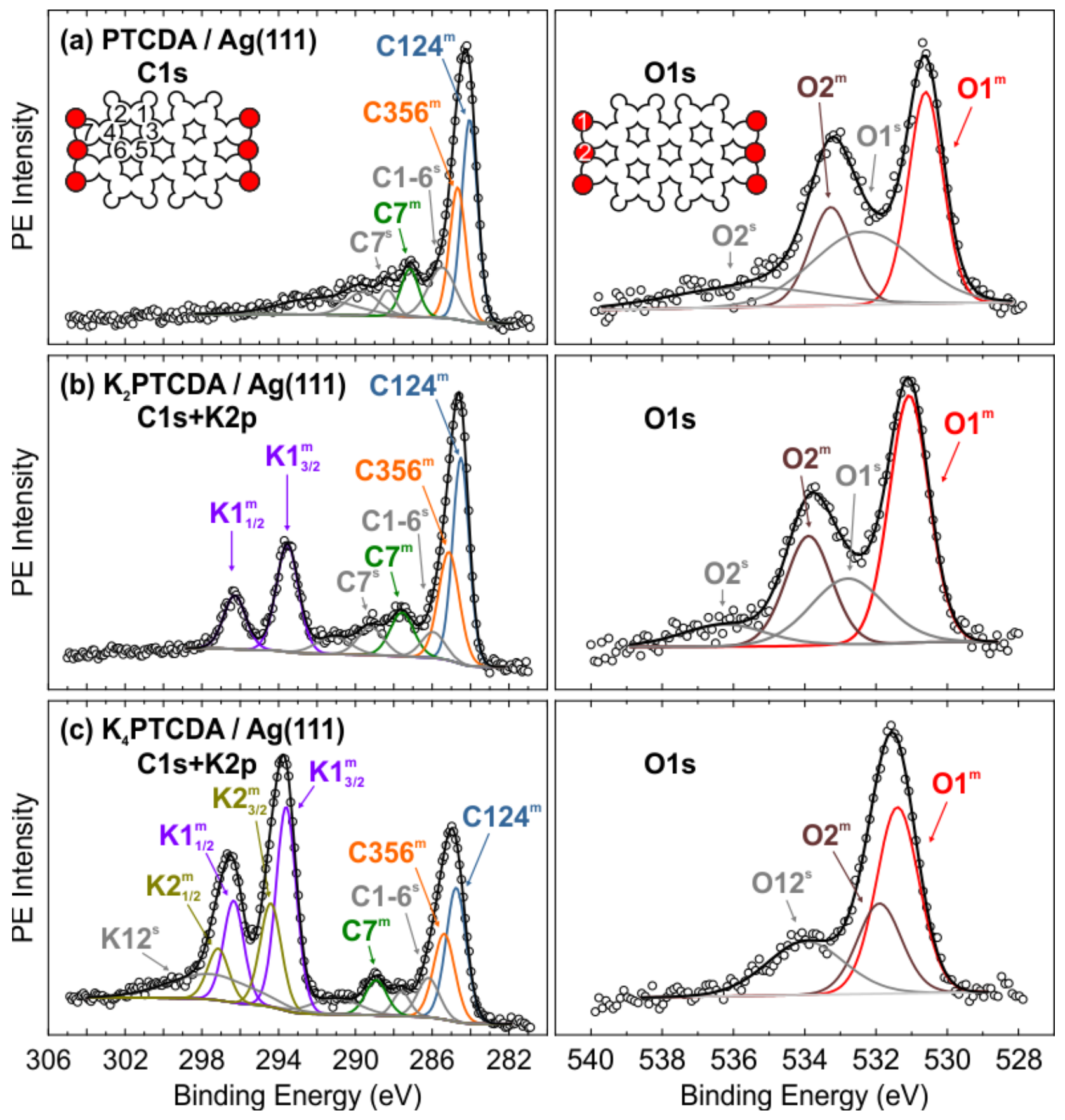

Figure S11: Core level spectra of (a) PTCDA/Ag(111), (b) $K_{2} P T C D A / A g(111)$ and (c) $K_{4}$ PTCDA/Ag(111). All spectra were acquired at $70^{\circ}$ emission using an excitation energy of $1486.7 \mathrm{eV}\left(\mathrm{Al} \mathrm{K} \mathrm{K}_{\alpha}\right)$. C1s and K2p data points are given as circles. The PE backgrounds of C1s and $K 2 p$ were described by Shirley functions, whereas linear backgrounds were used for O1s. The peaks of each individual component distinguished by the fitting model are shown as solid lines. The thick black lines represent the best fits to the data. 


\subsection{NIXSW Measurements}

For the NIXSW measurements, a film of 0.6 ML PTCDA was evaporated onto a clean Ag(111) surface using a dedicated molecular source. The coverage of this film was estimated by core level spectroscopy monitoring the intensity of the C1s emission line and comparing it to the corresponding intensity of a PTCDA monolayer film. Since DRS was not available for in situ monitoring the doping process at the beamline, we deposited potassium stepwise onto the PTCDA film while the corresponding structural changes were recorded with LEED. The deposition procedure was repeated until sharp LEED spots of the $\mathrm{K}_{2}$ PTCDA/Ag(111) phase emerged and the LEED spots of the PTCDA herringbone phase (PTCDA/Ag(111)) disappeared (cf. Fig. S12) ${ }^{9}$. However, by comparing our high-statistics (HS) C1s, K2p and O1s core level PE spectra (cf. Fig. S13) with the reference spectra of the pure phases (cf. Fig. S11), we obtained indications that the sample did not only contain $\mathrm{K}_{2}$ PTCDA but also $\mathrm{K}_{4}$ PTCDA complexes, though to a lesser extent. The stoichiometric ratio between both phases, however, cannot be determined reliably from our PE spectra as the different contributions overlap strongly. The estimated contents of potassium bonded to PTCDA molecules and excess potassium amount to 2.3 and 0.7 atoms per PTCDA molecule, respectively. In fact, the amount of potassium needed for the $\mathrm{K}_{2} \mathrm{PTCDA} / \mathrm{Ag}(111)$ formation has been exceeded, yet it was also insufficient to form a pure (highly ordered) $\mathrm{K}_{4} \mathrm{PTCDA} / \mathrm{Ag}(111)$ phase on the sample. We remind the reader that $\mathrm{K}_{2}$ PTCDA and $\mathrm{K}_{4}$ PTCDA can coexist on Ag(111), while $\mathrm{K}_{3}$ PTCDA is thermodynamically unstable and has not been observed. ${ }^{9}$ In order to account for the coexistence of $\mathrm{K}_{2} \mathrm{PTCDA}$ and $\mathrm{K}_{4} \mathrm{PTCDA}$ and for the higher photon energy $(h v=2620 \mathrm{eV}$ ) used for the experiments, we thus used the peak fitting model of the $K_{2}$ PTCDA phase (cf. Fig. S11b) and made the following modifications: The $C 7^{\mathrm{m}}$ peak of $\mathrm{K}_{4} \mathrm{PTCDA}$ and the $\mathrm{C} 7^{\mathrm{s}}$ peak of $\mathrm{K}_{2}$ PTCDA (cf. Fig. S11b,c) are found at almost the same binding energy and were accounted for here by only one peak labeled $\mathrm{C}^{\mathrm{m}+\mathrm{s}}$. Notice that the $\mathrm{C} 1 \mathrm{~s}$ main emission line includes contributions from both, $\mathrm{K}_{2}$ PTCDA and $\mathrm{K}_{4}$ PTCDA complexes. A new peak $\mathrm{O} 12^{\mathrm{m}+\mathrm{s}}$ is introduced for fitting the O1s spectrum in order to describe the contributions from $\mathrm{O}^{\mathrm{m}}$ of $\mathrm{K}_{4} \mathrm{PTCDA}$ together with contributions from $\mathrm{O}^{\mathrm{s}}$ of $\mathrm{K}_{2} \mathrm{PTCDA}$. We refrain from using a more comprehensive peak decomposition and more constraints since the stoichiometric ratio between $\mathrm{K}_{2} \mathrm{PTCDA}$ and $\mathrm{K}_{4} \mathrm{PTCDA}$ remains somewhat uncertain.

The derived fitting model for the HS-PE spectra was transferred to the XSW-PE spectra, which have a much lower signal-to-noise ratio. Therefore, we constrained the intensity ratio and the relative peak position of different species in the peak fitting procedure in order to increase its 
reliability. As a result, components with constrained peak areas cannot be analyzed independently in terms of their adsorption heights. This is the case for the main carbon peaks of the perylene core $\left(\mathrm{C} 124^{\mathrm{m}}, \mathrm{C} 356^{\mathrm{m}}\right.$, and C1-6 $\left.{ }^{\mathrm{s}}\right)$, which are constrained according to their stoichiometry. Consequently, a possible bending of the perylene core cannot be revealed directly anymore. This approach is feasible since the perylene core is rather rigid and the coherent fraction is fairly high as shown later. Thus, the obtained coherent parameters are largely independent from the specific peak fitting model chosen for the decomposition of the main emission line. Important parameters and constraints are compiled in Tab. S7.

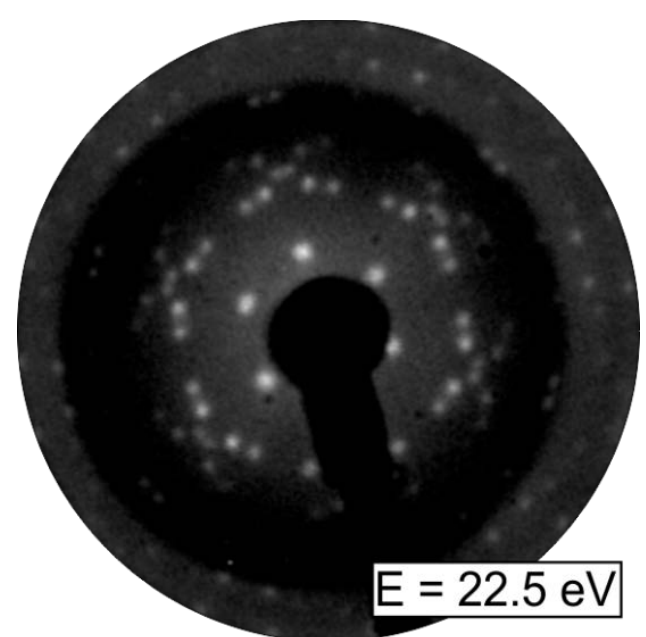

Figure S12: LEED image of the sample used for NIXSW investigations clearly and exclusively showing the pattern of $K_{2} P T C D A / A g(111)$ as investigated in detail in Ref. 9.
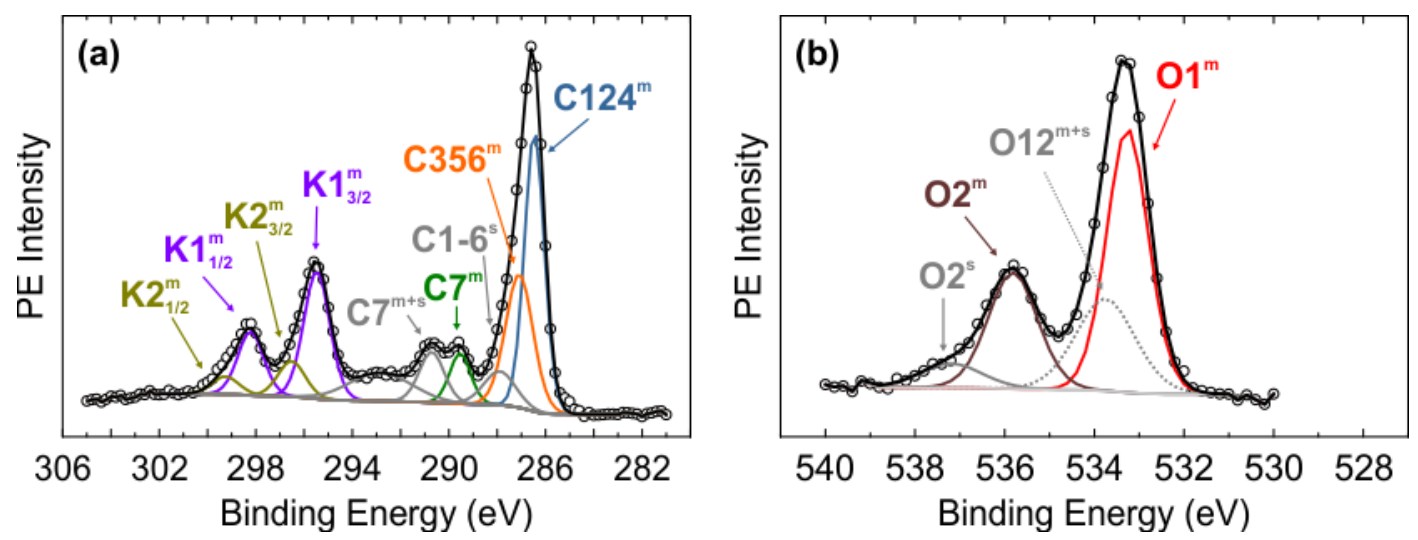

Figure S13: (a) C1s, K2p and (b) O1s high-statistics (HS) spectra of the K-doped PTCDA film, which were used for NIXSW investigations. All spectra were acquired at an emission angle close to $90^{\circ}$ with respect to the surface normal using an excitation energy of $2632 \mathrm{eV}$ (off-Bragg) and a pass energy of $100 \mathrm{eV}$. Measured data points are given as circles. The PE backgrounds of C1s and 
K2p were described by Shirley functions, whereas a linear background was used for O1s. Peaks are shown as solid lines. The thick black lines represent the best fits to the data.

Table S7: Peak parameters and constraints of the core level fitting model of the C1s, K2p and O1s signatures of K-doped PTCDA/Ag(111). $E_{B}$ : binding energy; FWHM: peak width; and relative peak areas with respect to the integrated PE yield of each element. Constraints are indicated by mathematical operations on components $(A, B, C, \ldots)$.

\begin{tabular}{|c|c|c|c|c|c|}
\hline Component & Peak & $\begin{array}{c}E_{B} \\
(e V)\end{array}$ & $\begin{array}{c}\text { FWHM } \\
(\mathrm{eV})\end{array}$ & $\begin{array}{l}\text { Area } \\
(\%)\end{array}$ & Origin \\
\hline$A$ & $\mathrm{C} 124^{\mathrm{m}}$ & 286.47 & 0.96 & 38.5 & \multirow{3}{*}{$\mathrm{C}_{\text {pery }}$} \\
\hline$B$ & $\mathrm{C} 356^{\mathrm{m}}$ & $A+0.62$ & 1.34 & $A \times 2 / 3$ & \\
\hline C & $\mathrm{C} 1-6^{\mathrm{S}}$ & $A+1.45$ & 1.54 & 7.5 & \\
\hline$D$ & $\mathrm{C} 7^{\mathrm{m}}$ & 289.55 & 1.02 & 7.4 & \multirow{2}{*}{$\mathrm{C}_{\text {func }}$} \\
\hline E & $\mathrm{C} 7^{\mathrm{m}+\mathrm{s}}$ & $D+1.18$ & 1.22 & 8.8 & \\
\hline$F$ & - & 292.66 & 3.08 & 12.2 & C satellite \\
\hline$G$ & $\mathrm{~K} 1_{3 / 2}^{\mathrm{m}}$ & $A+9.02$ & 1.26 & 51.5 & \multirow[b]{2}{*}{$\mathrm{K}_{\mathrm{PTCDA}}$} \\
\hline$H$ & $\mathrm{~K} 1_{1 / 2}^{\mathrm{m}}$ & $G+2.74$ & $G \times 1.0$ & $G \times 0.5$ & \\
\hline$I$ & $\mathrm{~K} 2_{3 / 2}^{\mathrm{m}}$ & $A+10.07$ & 1.31 & 15.1 & \multirow{2}{*}{$\mathrm{K}_{\text {excess }}$} \\
\hline$J$ & $\mathrm{~K} 2_{1 / 2}^{\mathrm{m}}$ & $I+2.74$ & $I \times 1.0$ & $I \times 0.5$ & \\
\hline K & $01^{\mathrm{m}}$ & 533.26 & 1.09 & 46.4 & $\mathrm{O}_{\text {carb }}$ \\
\hline$L$ & $012^{\mathrm{m}+\mathrm{s}}$ & 533.75 & 1.56 & 23.1 & $\mathrm{O}_{\text {carb }} \& \mathrm{O}_{\text {anhy }}$ \\
\hline$M$ & $\mathrm{O} 2^{\mathrm{m}}$ & 535.82 & 1.30 & 24.2 & 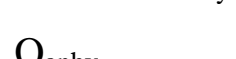 \\
\hline$N$ & $02^{\mathrm{s}}$ & $M+1.37$ & 1.62 & $M \times 0.26$ & anhy \\
\hline
\end{tabular}
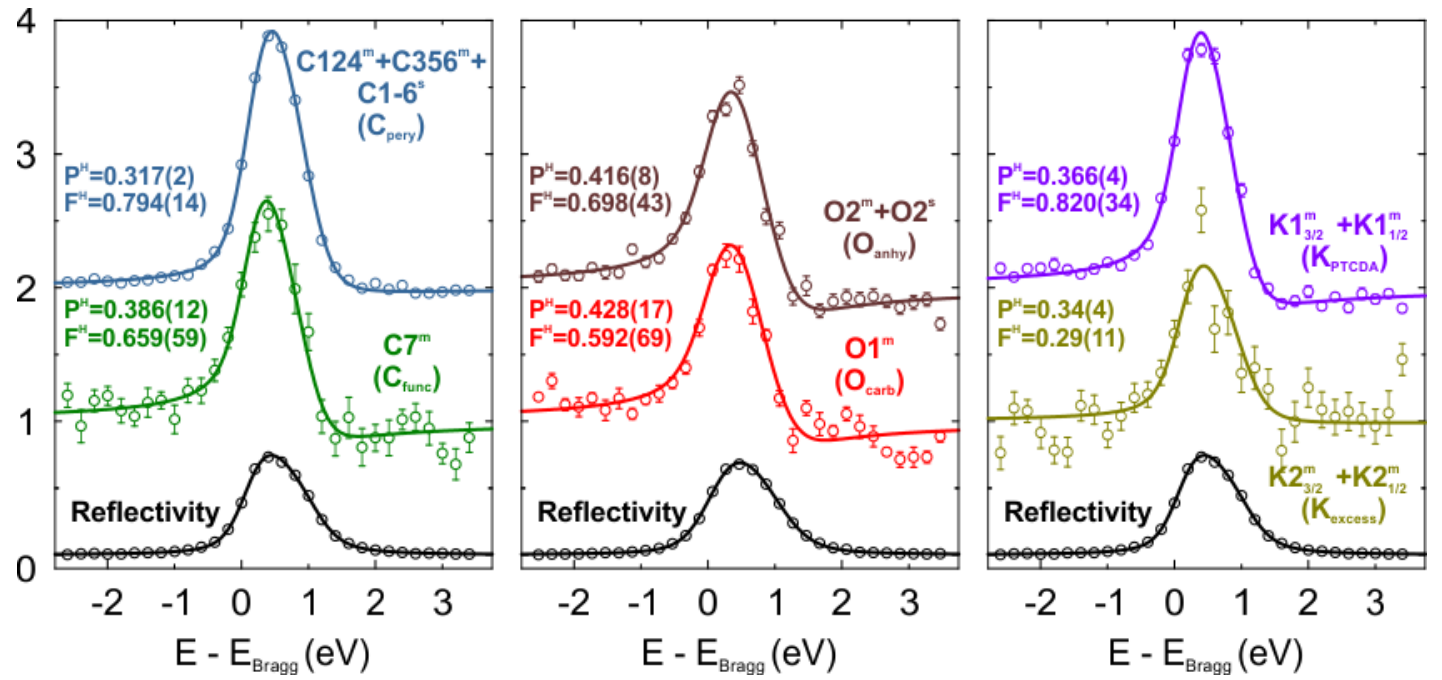

Figure S14: Partial yield curves obtained for a single NIXSW scan together with the reflectivity curves of the (111) Bragg reflection of the substrate. Measured data points are given as circles. 
The solid lines represent the best fit to the data according to XSW theory with the resulting parameters coherent fractions $F^{H}$ and coherent positions $P^{H}$. The error bars were calculated on the basis of the Monte Carlo simulation implemented in CasaXPS ${ }^{10}$.

Partial yield curves for all relevant chemical species of the K-doped PTCDA film were extracted from four NIXSW scans recorded at different sample positions. Typical partial yield curves are shown as data points in Fig. S14. The solid lines in the graphs represent least square fits to the data from which coherent fractions $\mathrm{F}^{\mathrm{H}}$ and coherent positions $\mathrm{P}^{\mathrm{H}}$ were obtained. All fitting results of all individual NIXSW scans are summarized in a polar diagram, the so called Argand diagram, in Fig. S15a, where $F^{H}$ and $P^{H}$ are represented by the polar angle $(0 \ldots 2 \pi)$ and absolute length $(0 \ldots 1)$ of a vector, respectively. The values averaged over all individual NIXSW scans for each atomic species are indicated as open circles. The adsorption heights were calculated according to: $d=$ $d_{111} \cdot\left(n+P^{H}\right)$ with the (111) lattice plane distance of Ag being $d_{111}=2.35 \AA$ and $n=1$. The averaged coherent parameters and adsorption heights are listed in Tab. S8 along with the respective errors. These errors purely refer to statistical uncertainties obtained from the data fitting procedures. Further systematic errors might have been introduced in the data analysis by (i) neglecting nondipolar effects and (ii) by the design of the peak fitting model, e.g., an imperfect decomposition of the chemically different components. We assume that contributions stemming from non-dipolar effects in our data are minor since the angle between the direction of the incident light and direction of photoelectron emission is almost $90^{\circ}$. Quantifying systematic errors in the peak fitting model is in general rather challenging. However, we are convinced that we have found the most precise model that can be obtained from the data available. Further, we have only used XPS peaks for the determination of adsorption heights that could be assigned unambiguously to the specific species, but we have also tested a wide variety of fitting models with different peak parameters and constraints. The model presented here is the one for which the highest coherent fractions could be achieved being a good indication that the partial XPS yields stemming from different species were separated successfully: The goodness of a fitting model can usually be estimated quite well by the obtained coherent fractions. When a large coherent fraction is obtained from the analysis of a certain XPS peak one can assume that the corresponding chemical species was well separated from any other inequivalent species, since averaging the electron yield from inequivalent species can only decrease, never increase the coherent fraction obtained. Hence, even if another parasitic species still contributes to the XPS peak in question, this species must be located at an equivalent adsorption height as the original one. In the case of a low coherent fraction the conclusion is less clear, but in case no multiple sites are expected for the species in question, a low fraction is a good 
indication for contributions from other parasitic species. ${ }^{11}$ The suggested vertical structure is shown in Fig. S15 as color-coded ball-and-stick side views together with that of undoped PTCDA/Ag(111) which is represented by gray spheres.

Table S8: Coherent parameters and adsorption heights of K-doped PTCDA monolayer domains overwhelmingly consisting of $K_{2} P T C D A$ on $A g(111)$. The values are the averaged results obtained from four individual NIXSW scans for each chemical species.

\begin{tabular}{lcccc}
\hline species & peaks & $\mathrm{P}^{\mathrm{H}}$ & $\mathrm{F}^{\mathrm{H}}$ & $\mathrm{z}(\AA)$ \\
\hline$C_{\text {pery }}$ & $\mathrm{C} 124^{\mathrm{m}}+\mathrm{C} 356^{\mathrm{m}}+\mathrm{C} 1-6^{\mathrm{s}}$ & $0.323(6)$ & $0.73(5)$ & $3.12(2)$ \\
$C_{\text {func }}$ & $\mathrm{C}^{\mathrm{m}}$ & $0.38(3)$ & $0.62(14)$ & $3.26(7)$ \\
$K_{\text {PTCDA }}$ & $\mathrm{K} 1_{3 / 2}^{\mathrm{m}}+\mathrm{K} 1_{1 / 2}^{\mathrm{m}}$ & $0.367(11)$ & $0.76(7)$ & $3.23(3)$ \\
$K_{\text {excess }}$ & $\mathrm{K} 2_{3 / 2}^{\mathrm{m}}+\mathrm{K} 2_{1 / 2}^{\mathrm{m}}$ & $0.38(13)$ & $0.23(20)$ & $3.26(30)$ \\
$O_{\text {carb }}$ & $\mathrm{O} 1^{\mathrm{m}}$ & $0.423(30)$ & $0.61(11)$ & $3.36(7)$ \\
$O_{\text {anhy }}$ & $\mathrm{O} 2^{\mathrm{m}}+\mathrm{O} 2^{\mathrm{s}}$ & $0.425(20)$ & $0.650(88)$ & $3.36(5)$ \\
\hline
\end{tabular}

(a)

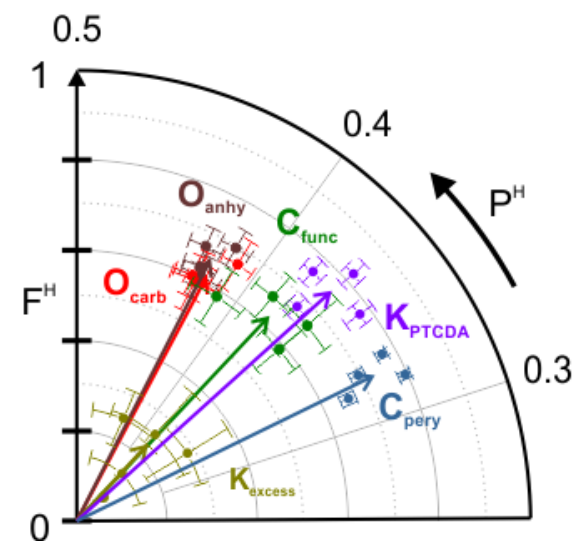

(b)

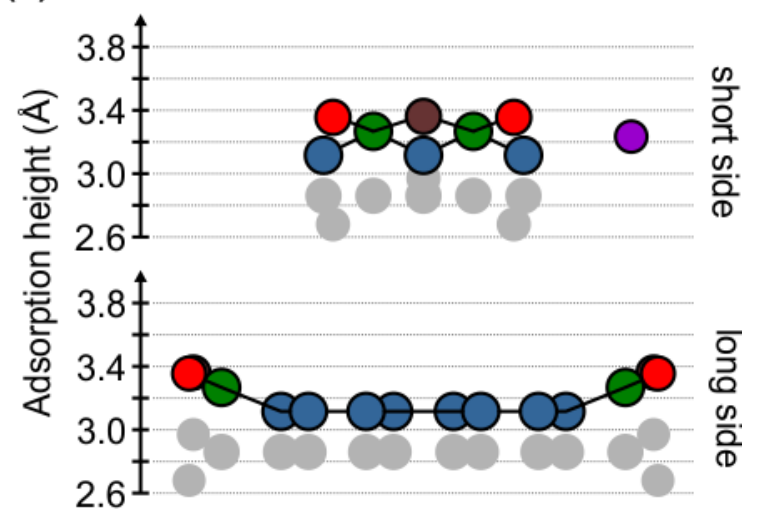

Figure S15: Graphical representation of the results compiled in Table S8 for $K_{2} P T C D A / A g(111)$. (a) Argand diagram and (b) suggested vertical adsorption geometry of PTCDA and potassium. The coherent parameters of four single NIXSW measurements for each species are shown as data points in the Argand diagram. The error bars are standard deviations of the fits of the partial yield curves. The vectors point to the averaged values obtained for each species. The color code in (a) and (b) is the same as used in Fig. S14. Gray spheres in (b) represent previously published data of the undoped monolayer of PTCDA/Ag(111) for comparison. ${ }^{12}$ 


\section{References}

(1) Fratesi, G.; Motta, C.; Trioni, M. I.; Brivio, G. P.; Sánchez-Portal, D. Resonant Lifetime of Core-Excited Organic Adsorbates from First Principles. J. Phys. Chem. C 2014, 118, 87758782.

(2) Hanwell, M. D.; Curtis, D. E.; Lonie, D. C.; Vandermeersch, T.; Zurek, E.; Hutchison, G. R. Avogadro: An Advanced Semantic Chemical Editor, Visualization, and Analysis Platform. $J$. Cheminform. 2012, 4, 17.

(3) Forker, R.; Gruenewald, M.; Fritz, T. Optical Differential Reflectance Spectroscopy on Thin Molecular Films. Annu. Rep. Prog. Chem. Sect. C: Phys. Chem. 2012, 108, 34-68.

(4) Selci, S.; Ciccacci, F.; Chiarotti, G. Surface Differential Reflectivity Spectroscopy of Semiconductor Surfaces. J. Vac. Sci. Technol. 1987 ,5, 327-332.

(5) Jiang, Y.; Pillai, S.; Green, M. Realistic Silver Optical Constants for Plasmonics. Sci. Rep. 2016, 6, 30605.

(6) Schöll, A.; Zou, Y.; Jung, M.; Schmidt, T.; Fink, R.; Umbach, E. Line Shapes and Satellites in High-Resolution X-Ray Photoelectron Spectra of Large $\pi$-Conjugated Organic Molecules. $J$. Chem. Phys. 2004, 121, 10260-10267.

(7) Fratesi, G.; Lanzilotto, V.; Stranges, S.; Alagia, M.; Brivio, G. P.; Floreano, L. High Resolution NEXAFS of Perylene and PTCDI: A Surface Science Approach to Molecular Orbital Analysis. Phys. Chem. Chem. Phys. 2014, 16, 14834-14844.

(8) Xu, L.; Meng, Y.; Shi, Y.; Liu, Y. Pitting Corrosion of 13Cr Steel in Oxygen-Free Completion Fluids of Organic Salt. Acta Met. Sin. Engl. Lett. 2013, 26, 271-276.

(9) Zwick, C.; Baby, A.; Gruenewald, M.; Verwüster, E.; Hofmann, O. T.; Forker, R.; Fratesi, G.; Brivio, G. P.; Zojer, E.; Fritz, T. Complex Stoichiometry-Dependent Reordering of 3,4,9,10Perylenetetracarboxylic Dianhydride on Ag(111) upon K Intercalation. ACS Nano 2016, 10, 2365-2374.

(10) Mercurio, G.; Bauer, O.; Willenbockel, M.; Fairley, N.; Reckien, W.; Schmitz, C. H.; Fiedler, B.; Soubatch, S.; Bredow, T.; Sokolowski, M.; Tautz F. S. Adsorption Height Determination of Nonequivalent C and O Species of PTCDA on Ag(110) Using X-Ray Standing Waves. Phys. Rev. B: Condens. Matter Mater. Phys. 2013, 87, 45421.

(11) Stadtmüller, B.; Willenbockel, M.; Schröder, S.; Kleimann, C.; Reinisch, E. M.; Ules, T.; Soubatch, S.; Ramsey, M. G.; Tautz, F. S.; Kumpf, C. Modification of the PTCDA-Ag Bond by Forming a Heteromolecular Bilayer Film. Phys. Rev. B: Condens. Matter Mater. Phys. 2015, 91, 155433.

(12) Hauschild, A.; Temirov, R.; Soubatch, S.; Bauer, O.; Schöll, A.; Cowie, B. C. C.; Lee, T.-L.; Tautz, F. S.; Sokolowski, M. Normal-Incidence X-Ray Standing-Wave Determination of the Adsorption Geometry of PTCDA on Ag(111): Comparison of the Ordered RoomTemperature and Disordered Low-Temperature Phases. Phys. Rev. B: Condens. Matter Mater. Phys. 2010, 81, 125432. 